Keywords: Tank Farm, Tank

Closure, Tank 6F

Retention: Permanent

\title{
Analysis of the Tank 6F Final Characterization Samples-2012
}

L. N. Oji, D. P. Diprete, C. J. Coleman, M. S. Hay and E. P. Shine

June 2012

Savannah River National Laboratory Savannah River Nuclear Solutions Aiken, SC 29808

Prepared for the U.S. Department of Energy under contract number DE-AC09-08SR22470.

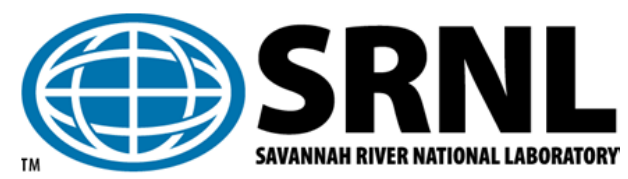




\section{DISCLAIMER}

This work was prepared under an agreement with and funded by the U.S. Government. Neither the U.S. Government or its employees, nor any of its contractors, subcontractors or their employees, makes any express or implied:

1. warranty or assumes any legal liability for the accuracy, completeness, or for the use or results of such use of any information, product, or process disclosed; or

2. representation that such use or results of such use would not infringe privately owned rights; or

3. endorsement or recommendation of any specifically identified commercial product, process, or service.

Any views and opinions of authors expressed in this work do not necessarily state or reflect those of the United States Government, or its contractors, or subcontractors.

Printed in the United States of America

Prepared for

U.S. Department of Energy 
AUTHORS:

\section{REVIEWS AND APPROVALS}

L. N. Oji, Author, Advanced Characterization \& Processing

Date

D. P. Diprete, Co-author, Analytical Development

Date

C. J. Coleman, Co-author, Analytical Development

Date

M.S. Hay, Co-author, Advanced Characterization \& Processing

Date

E. P. Shine, Co-author, Applied Comp. Engineering \& Statistics

Date

\section{TECHNICAL REVIEW:}

S. H. Reboul, Process Technology Programs

Date

T. B. Edwards, Applied Comp. Engineering \& Statistics

Date

\section{APPROVALS:}

F. M. Pennebaker, Manager, Advanced Characterization \& Processing

Date

P. L. Lee, Manager, Applied Comp. Engineering \& Statistics

Date

S. L. Marra, Manager,

Date

Environmental \& Chemical Process Technology Research Programs

M. J. Mahoney, Closure \& Waste Disposal Authority

Date

A. J. Tisler, Manager, SRR Engineering

Date 


\section{EXECUTIVE SUMMARY}

\section{Characterization summary}

The Savannah River National Laboratory (SRNL) was requested by Savannah River Remediation (SRR) to provide sample preparation and analysis of the Tank $6 \mathrm{~F}$ final characterization samples to determine the residual tank inventory prior to grouting. Fourteen residual Tank 6F solid samples from three areas on the floor of the tank were collected and delivered to SRNL between May and August 2011.

These Tank 6F samples were homogenized and combined into three composite samples based on a proportion compositing scheme and the resulting composite samples were analyzed for radiological, chemical and elemental components. Additional measurements performed on the Tank 6F composite samples include bulk density and water leaching of the solids to account for water soluble components. The composite Tank $6 \mathrm{~F}$ samples were analyzed and the data reported in triplicate.

Sufficient quality assurance standards and blanks were utilized to demonstrate adequate characterization of the Tank $6 \mathrm{~F}$ samples. The main evaluation criteria were target detection limits specified in the technical task request document. While many of the target detection limits were met for the species characterized for Tank $6 \mathrm{~F}$ some were not met. In a few cases, the relatively high levels of radioactive species of the same element or a chemically similar element precluded the ability to measure some isotopes to low levels. The isotopes whose detection limits were not met in all cases included Sn-126, Sb-126, Sb-126m, Eu-152, Cm243 and Cf-249. SRNL, in conjunction with the customer, reviewed all of these cases and determined that the impacts of not meeting the target detection limits were acceptable.

\section{Statistical review summary}

Based on the analyses of variance (ANOVA) for the inorganic constituents of Tank $6 \mathrm{~F}$, all the inorganic constituents displayed heterogeneity. The inorganic results demonstrated consistent differences across the composite samples: lowest concentrations for Composite Sample 1, intermediate-valued concentrations for Composite Sample 2, and highest concentrations for Composite Sample 3. The $\mathrm{Hg}$ and $\mathrm{Mo}$ results suggest possible measurement outliers. However, the magnitudes of the differences between the $\mathrm{Hg} 95 \%$ upper confidence limit (UCL95) results with and without the outlier and the magnitudes of the differences between the Mo UCL95 results with and without the outlier do not appear to have practical significance. It is recommended to remove the potential measurement outliers. Doing so is conservative in the sense of producing a higher UCL95 for Hg and Mo than if the potential outliers were included in the calculations.

In contrast to the inorganic results, most of the radionuclides did not demonstrate heterogeneity among the three Tank 6F composite sample characterization results. 


\section{TABLE OF CONTENTS}

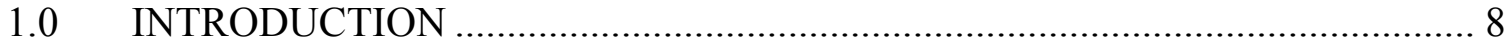

2.0 SAMPLING AND SAMPLE PREPARATION FOR CHARACTERIZATION. 11

2.1 Blank Evaluations and Reference Materials ..................................................... 15

2.2 Leaching Characterization of Tank 6F Solids ................................................. 15

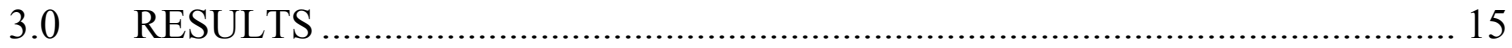

3.1 Data Quality and Presentations for Routine Radionuclide Constituents .............. 17

3.2 Data Quality and Presentations for Elemental Constituents ................................ 18

3.3 Data Quality and Presentations for Non-Routine Radionuclide Analytes........... 19

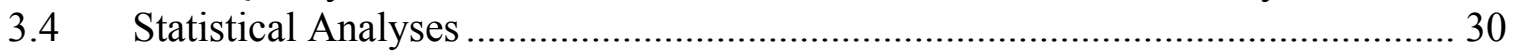

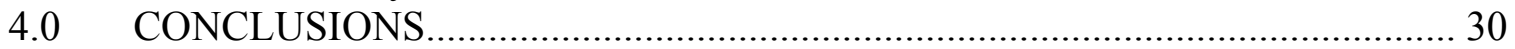

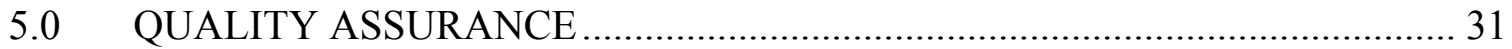

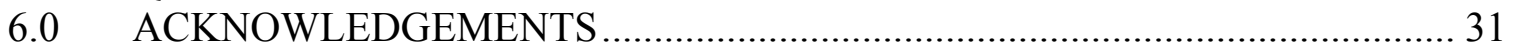

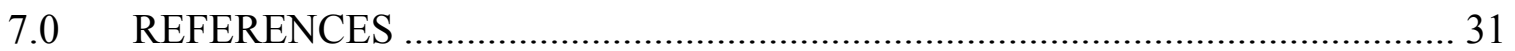

Appendix A-1 AD Tracking Numbers for Tank 6F Characterization .............................. 32

Appendix A-2 AD Tracking Numbers for Tank 6F Characterizations -Continued........... 33

Appendix A-3. Chemical Composition for Reference Tank 8 Simulant Sludge ............... 33

Appendix A-4. Chemical Composition of Analyzed Reference Glass................................ 34

Appendix A-5. ICPES and ICP-MS Comparisons for Select Analytes............................. 35

Appendix B - Summary of Analytical Methods …………………………………….... 37

Appendix C Summary of Statistical Methods .................................................................. 44

\section{LIST OF TABLES}

Table 1 Tank 6 Sample ID Numbers and Composite Sample Proportions............................ 12

Table 2 Tank 6F “As-Received” Sample Bulk Density*, g/mL ............................................... 12

Table 3 Tank 6F "Homogenized” Sample Bulk Density, g/mL .............................................. 13

Table 4 Tank 6F Composite Sample Bulk Density, g/mL ................................................ 13

Table 5 Tank 6F Sample Compositions for Composite Samples 1, 2 and 3........................ 14

Table 6 Weight Percent Solids for Tank 6F Composite Samples, Wt\% …........................... 14

Table 7 Weight Percent Anions Leached per gram of Tank 6F- Composite Sample \#1 ....... 21

Table 8 Weight Percent Anions Leached per gram of Tank 6F- Composite Sample \#2 2....... 21

Table 9 Weight Percent Anions Leached per gram of Tank 6F- Composite Sample \#3 ........ 21

Table 10 Elemental Constituents in Tank 6F Composite Sample \# 1, wt \% .......................... 22

Table 11 Elemental Constituents in Tank 6F Composite Sample \# 2, wt \% ......................... 23

Table 12 Elemental Constituents in Tank 6F Composite Sample \# 3, wt\% ……................... 24

Table 13 Radiological Constituents for Tank 6F Composite Sample \#1, $\mu \mathrm{Ci} / \mathrm{g}$..................... 25

Table 14 Radiological Constituents for Tank 6F Composite Sample \#2, $\mu \mathrm{Ci} / \mathrm{g}$.................. 27

Table 15 Radiological Constituents for Tank 6F Composite Sample \#3, $\mu \mathrm{Ci} / \mathrm{g}$................... 29

Table 16 Tank 6F Constituents for Statistical Evaluation ..................................................... 45

Table 17 Tests and Estimators for Density, Weight Percent, and Anions .............................. 56

Table 18 Anion and Tritium Concentrations less than their MDCs ...................................... 59

Table 19 95\% UCLs for Mean Density, Weight Percent Solids, and Anion Concentrations

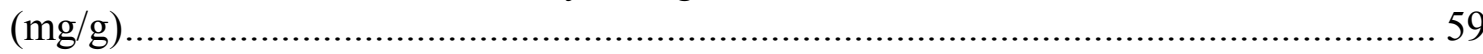


Table 20 Tests and Estimators for Inorganic Constituents 60

Table 21 Computations for the Mean, Standard Error, and the 95\% Upper Confidence Limit for the Mean Hg Concentration Omitting the Result for Run 1 of Composite Sample 170 Table 22 Computations for the Mean, Standard Error, and the 95\% Upper Confidence Limit for the Mean Mo Concentration Omitting the Result for Run 1 of Composite Sample 3

Table 23 Inorganic Constituent Concentrations with All Results Less Than Their MDCs... 72 Table 24 95\% UCLs for Mean Concentrations of Inorganic Constituents with All Results

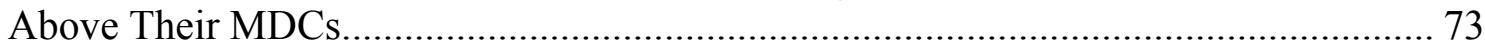

Table 25 Tests and Estimators for Radionuclides............................................................ 74

Table 26 Radionuclide Concentrations with All Results below Their MDCs..................... 92 Table 27 95\% UCLs for Mean Concentrations of Radionuclide Constituents with All Results

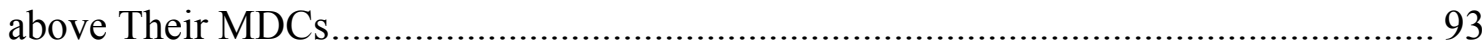

\section{LIST OF FIGURES}

Figure 1: Tank 6F Sample Locations ............................................................................ 9

Figure 2 Photo images of some "as-received" Tank 6F samples....................................... 10

Figure 3. Sequence of Goodness-of-Fit Tests to Identify a Distribution and Select an

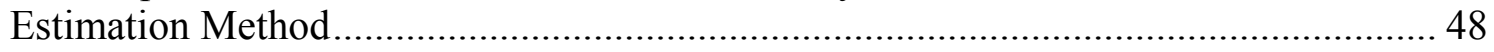

Figure 4. Scatterplots for the Inorganic Constituents .................................................. 51

Figure 5. Scatterplots for a Subset of the Radionuclides .................................................... 53 


\section{LIST OF ABBREVIATIONS}

$\begin{array}{ll}\text { AD } & \text { Analytical Development } \\ \text { ANOVA } & \text { Analyses of Variance } \\ \text { ARG } & \text { Analyzed Reference Glass } \\ \text { AQR } & \text { Aqua Regia Digestions } \\ \text { C\&WDA } & \text { Closure and Disposal Authority } \\ \text { DL } & \text { Detection limit: As used in mass spectrophotometer analyses or ICP- } \\ \text { ICP-ES } & \text { ES; three times the standard deviation of the blank measurements. } \\ \text { ICP-MS } & \text { Inductively Coupled Plasma-Atomic Emission Spectrkoscopy } \\ \text { LIMS } & \text { Laboratory Information Management System } \\ \text { LWO } & \text { Liquid Waste Operations } \\ \text { MDA } & \text { Minimum Detectable Activity: Minimum detectable activity is the } \\ \text { PF } & \text { value above which instrument signal can be considered real. } \\ \text { PMP } & \text { Sodium Peroxide/Hydroxide Fusions } \\ \text { PuTTA } & \text { Polymethyl Pentane } \\ \text { UCL95 } & \text { Plutonium thenoyltrifluoro-acetone } \\ \text { UL } & \text { Upper 95\% Confidence Limit } \\ \text { SRNL } & \text { Upper limit: Activity observed but biased high due to spectral } \\ \text { SRR } & \text { interference or blank contamination. } \\ \text { TTQAP } & \text { Savannah River National Laboratory } \\ \text { TTR } & \text { Savannah River Remediation } \\ & \text { Task Technical Quality Assurance Plan } \\ \text { Technical Task Request }\end{array}$




\subsection{INTRODUCTION}

Savannah River Remediation (SRR) is preparing Tank 6F for closure. The Savannah River National Laboratory (SRNL) was requested by SRR to provide sample preparation and analysis of the Tank $6 \mathrm{~F}$ final characterization samples to determine the residual tank inventory prior to grouting. In all, fourteen floor samples from three areas in Tank $6 \mathrm{~F}$ were provided by SRR. These Tank $6 \mathrm{~F}$ samples were taken from the tank and made available to SRNL between May and August 2011. Figure 1 shows the three locations in the Tank where these samples were taken. A photo image of some of the "as-received" Tank $6 \mathrm{~F}$ samples is shown in Figure 2.

The fourteen Tank $6 \mathrm{~F}$ samples formed the basis for designing the three Tank $6 \mathrm{~F}$ composite materials (Tank 6F-Composite sample \# 1, Tank 6F-Composite sample \# 2 and Tank 6FComposite sample \# 3). The volume of residual material in each of the Tank $6 \mathrm{~F}$ regions was obtained by SRR and this information was used to estimate the strata volumes in the tank. These strata volumes were converted into volumetric proportions and subsequently to the mass of residual material to be obtained from each primary sample for each composite sample ${ }^{1}$ as summarized in Table 1 .

The Tank $6 \mathrm{~F}$ samples were analyzed in accordance with $\mathrm{TTR}^{6}$, and $\mathrm{TTQAP}^{7}$, for the analysis of Tank $5 \mathrm{~F}$ and $6 \mathrm{~F}^{7}$ and Tank $6 \mathrm{~F}$ Sampling analysis plan ${ }^{8}$. The Justification for Changes to the Tank 6 Sampling and Analysis Plan, SRR-CWDA-2011-00159, Nov., 2011, Rev.0. ${ }^{2}$

The scope of this work includes characterization of Tank 6F composite samples and statistical analyses of select data as specified in the $\mathrm{TTR}^{6}$. 


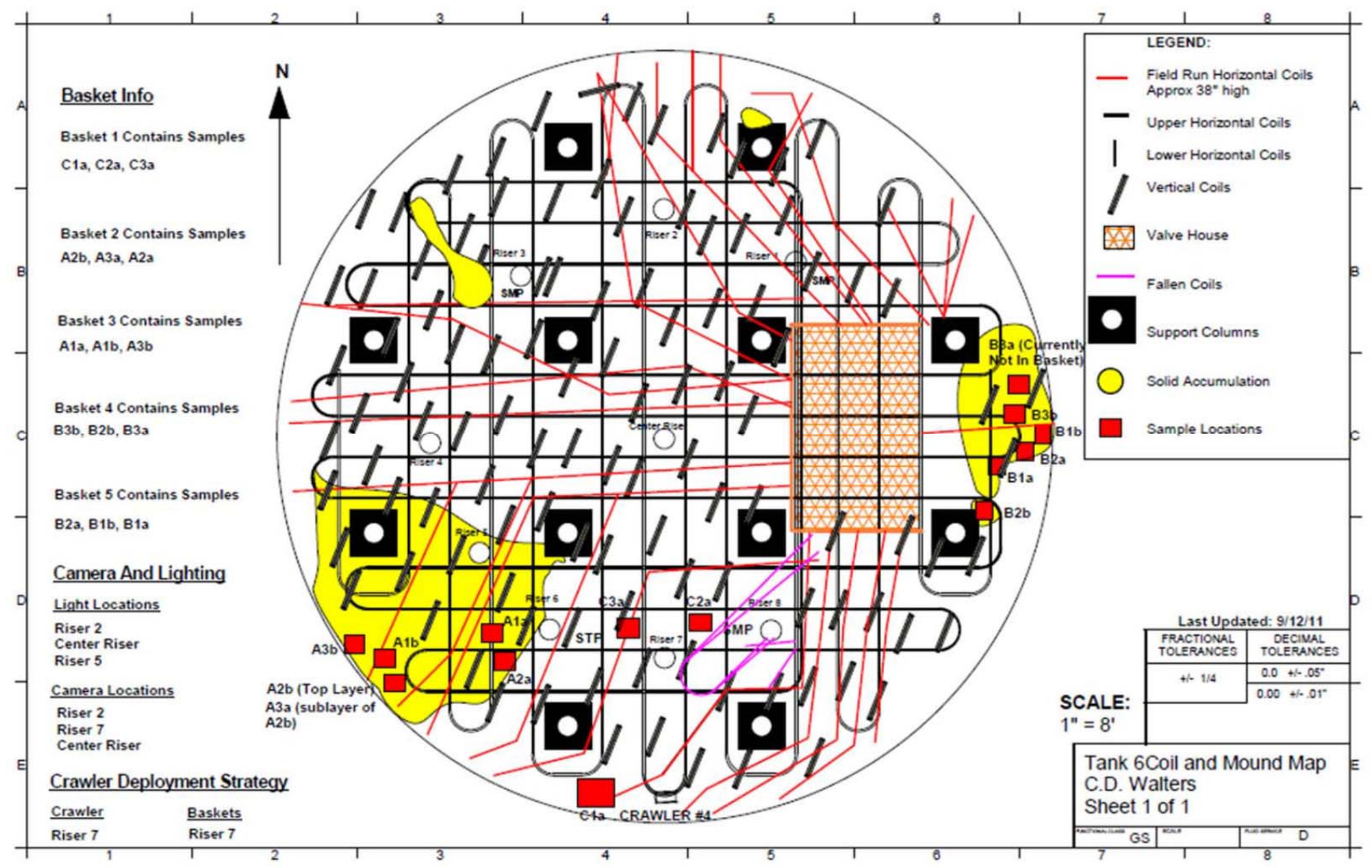

Figure 1 Tank 6F Sample Locations 
SRNL-STI-2012-00365, Rev. 0

Page 10 of 94

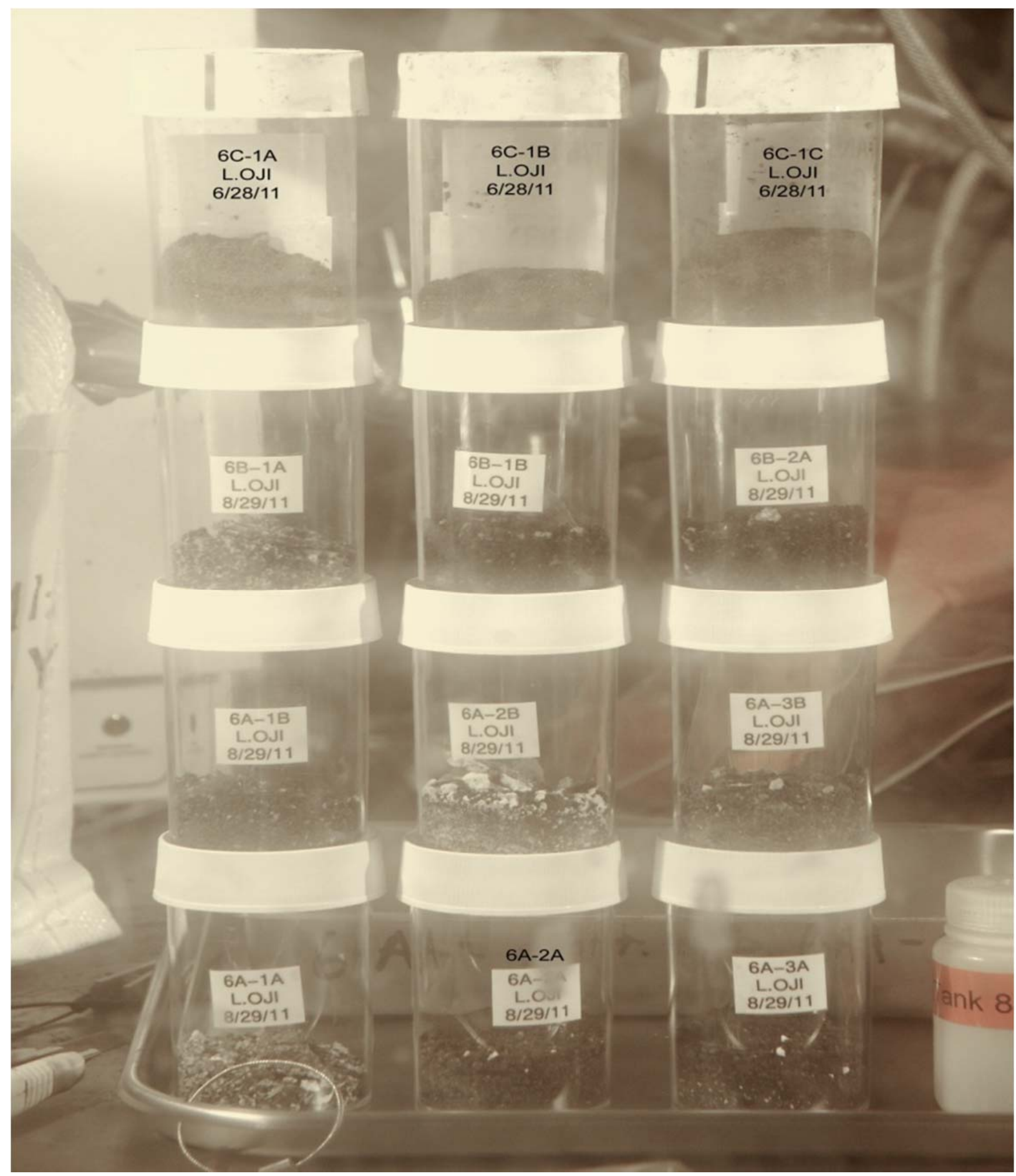

Figure 2 Photo images of some "as-received" Tank 6F samples. 


\subsection{SAMPLING AND SAMPLE PREPARATION FOR CHARACTERIZATION}

Samples from planned locations along the north side of the solids accumulation under riser 5 in Tank 6F were actually collected from the central/south region. This was determined by SRR to be acceptable because of process history and because the solids appearances in the tank photos indicate that the Tank $6 \mathrm{~F}$ solids were well-mixed. Thus, actual samples are representative of the entire solids accumulation under Riser 5. Five of the planned six samples were collected from the solids accumulation along the east edge of Tank 6F because the sample crawler became hung up on a cooling coil and could not return the last sample to the collection basket for retrieval from the tank. As a solution to the unattained sample, SRR decided to take additional volume from one of the five samples to adequately represent the east accumulation in one of the three composite samples. The impact on analysis results uncertainty was accessed to be acceptable given the number of samples used for each composite and given that the single sample from the east accumulation is limited to only one of the three composite samples. Therefore, C\&WDA and SRR Engineering determined that the minimal benefit of taking additional samples in Tank $6 \mathrm{~F}$ did not warrant the potential worker exposure and risk of contamination. ${ }^{2}$

A few of the Tank 6F samples were moist and needed further air drying in the shielded cells before preparation for compositing. The individual "as-received" materials were weighed and their "as-received" dry bulk densities determined prior to preparing each sample for composition and characterization. Each Tank 6F sample was then homogenized to promote particle size reduction due to the presence of chunks of solids. Homogenizing each sample involved grinding with a mortar and pestle and then passing the powder through a sieve with 850 micron openings (mesh 18). Materials which did not go through the sieve were ground with mortar and pestle until it was small enough to go through the sieve. The bulk density of each homogenized sample was determined followed by the blending of proportional amounts of the samples by weight to form three composite Tank 6F samples. The bulk density of each of the three composite samples was then determined by the process described in Appendix B. A reference simulant sludge sample, based on Tank 8 sample chemistry [See Appendix A-3], was air-dried in a clean laboratory and the resulting sludge cake ground and homogenized with a mortar and pestle. The bulk density of this reference Tank 8 sludge was determined both inside the shielded cell along with the Tank 6F samples and outside the cell in a clean laboratory hood. These Tank 8 sludge simulant bulk density values were used to verify how well the reference bulk densities could be reproduced both inside (using remote handling via manipulators) and outside of the shielded cell.

SRR determined the composite sample volumetric percent as shown in Table 1, B. Dean, "Tank 6F Composite Sample Volumetric Proportions," SRR-CWDA-2011-00067, Rev.1 April 20, 2011'. All bulk density data for the "as-received", homogenized and composited Tank 6F samples are presented in Tables 2 through 4 . The weight percent solid determination method is described in Appendix B. These individual sample proportional location volumes, along with the homogenized sample bulk densities were used to calculate each sample's mass per composite volume (Table 5). The total provided the composite density and the required weight from each of the fourteen Tank 6F sample material needed to make the three 70-gram composite Tank 6F samples as shown in Table 5. The weight percent solids in the three composite Tank 6F samples are summarized in Table 6. 
Because of the inherent risk of cross-contamination of these samples in the shielded cells environment, certain actions were taken to minimize the risks. Actions taken to control crosscontamination in the cell included wiping down the cell (cell decontaminated), covering the entire cell floor with clean stainless steel plates, and changing manipulator fingers prior to initiating work.

Table 1 Tank 6 Sample ID Numbers and Composite Sample Proportions

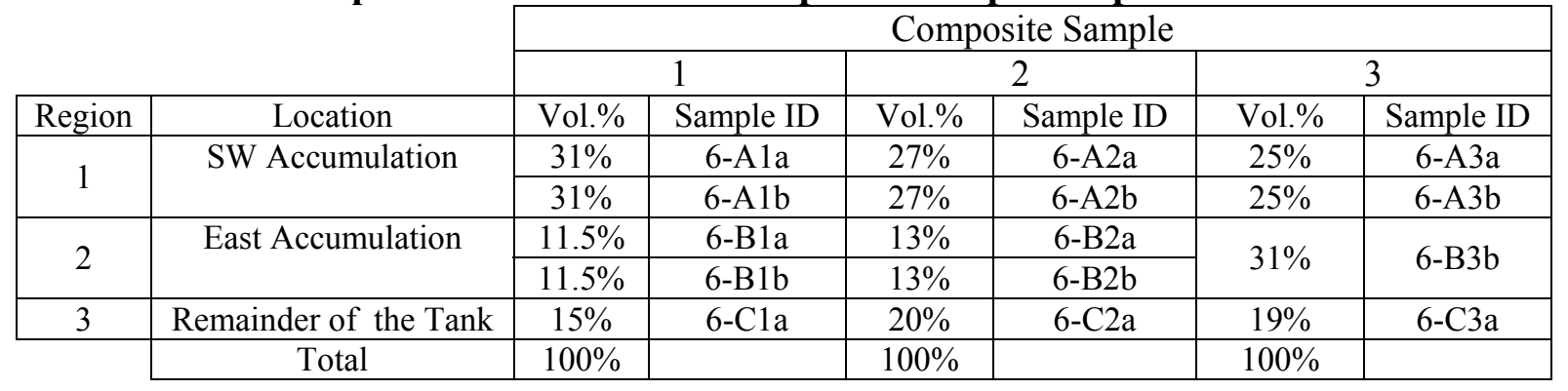

Table 2 Tank 6F “As-Received" Sample Bulk Density*, g/mL

\begin{tabular}{|c|c|c|c|c|c|}
\hline Tank 6F Sample ID & Run-1 & Run-2 & Run-3 & Average & Stdev. \\
\hline TK6-A1a & 1.17 & 1.19 & 1.18 & $\mathbf{1 . 1 8}$ & 0.01 \\
\hline TK 6-B1a & 1.56 & 1.51 & 1.58 & $\mathbf{1 . 5 5}$ & 0.04 \\
\hline TK 6-B1b & 1.66 & 1.65 & 1.56 & $\mathbf{1 . 6 2}$ & 0.06 \\
\hline TK 6-A1b & 1.30 & 1.35 & 1.32 & $\mathbf{1 . 3 2}$ & 0.03 \\
\hline TK 6-C1a & 1.07 & 1.09 & 1.07 & $\mathbf{1 . 0 8}$ & 0.01 \\
\hline TK 6-B2a & 1.55 & 1.69 & 1.70 & $\mathbf{1 . 6 5}$ & 0.08 \\
\hline TK 6-A2a & 1.69 & 1.58 & 1.62 & $\mathbf{1 . 6 3}$ & 0.06 \\
\hline TK 6-A2b & 1.42 & 1.36 & 1.40 & $\mathbf{1 . 3 9}$ & 0.03 \\
\hline TK 6-B2b & 1.09 & 1.28 & 1.20 & $\mathbf{1 . 1 9}$ & 0.10 \\
\hline TK 6-C2a & 1.12 & 1.13 & 1.20 & $\mathbf{1 . 1 5}$ & 0.04 \\
\hline TK 6-B3a & No sample & No sample & No sample & No sample & \\
\hline TK 6-B3b & 0.87 & 0.85 & 0.84 & $\mathbf{0 . 8 5}$ & 0.02 \\
\hline TK 6-A3a & 1.46 & 1.47 & 1.39 & $\mathbf{1 . 4 4}$ & 0.05 \\
\hline TK 6-A3b & 1.52 & 1.51 & 1.47 & $\mathbf{1 . 5 0}$ & 0.03 \\
\hline TK 6-C3a & 1.14 & 1.15 & 1.12 & $\mathbf{1 . 1 4}$ & 0.02 \\
\hline Tk 8 simulant sludge & 1.40 & 1.45 & 1.43 & $\mathbf{1 . 4 3}$ & 0.03 \\
\hline
\end{tabular}

* The "As-received" density values may in some cases have large uncertainty values. Problems were encountered in determining the volumes of these samples in calibrated PMP beakers. Most of the samples contained large chunky pieces, which made it difficult to determine acceptable sample volumes. 
Table 3 Tank 6F "Homogenized" Sample Bulk Density, g/mL

\begin{tabular}{|c|c|c|c|c|c|}
\hline Tank 6F Sample ID & Run-1 & Run-2 & Run-3 & Average & Stdev. \\
\hline TK6-A1a & 1.12 & 1.16 & 1.29 & $\mathbf{1 . 1 9}$ & 0.09 \\
\hline TK 6-B1a & 1.38 & 1.30 & 1.34 & $\mathbf{1 . 3 4}$ & 0.04 \\
\hline TK 6-B1b & 1.17 & 1.15 & 1.16 & $\mathbf{1 . 1 6}$ & 0.01 \\
\hline TK 6-A1b & 1.54 & 1.58 & 1.51 & $\mathbf{1 . 5 5}$ & 0.04 \\
\hline TK 6-C1a & 1.07 & 1.09 & 1.07 & $\mathbf{1 . 0 8}$ & 0.01 \\
\hline TK 6-B2a & 1.30 & 1.23 & 1.24 & $\mathbf{1 . 2 6}$ & 0.03 \\
\hline TK 6-A2a & 1.50 & 1.41 & 1.42 & $\mathbf{1 . 4 4}$ & 0.05 \\
\hline TK 6-A2b & 1.40 & 1.28 & 1.35 & $\mathbf{1 . 3 4}$ & 0.06 \\
\hline TK 6-B2b & 1.35 & 1.32 & 1.30 & $\mathbf{1 . 3 2}$ & 0.03 \\
\hline TK 6-C2a & 1.12 & 1.13 & 1.20 & $\mathbf{1 . 1 5}$ & 0.04 \\
\hline TK 6-B3a & No sample & No sample & No sample & No sample & \\
\hline TK 6-B3b & 1.29 & 1.31 & 1.30 & $\mathbf{1 . 3 0}$ & 0.01 \\
\hline TK 6-A3a & 1.38 & 1.31 & 1.26 & $\mathbf{1 . 3 2}$ & 0.06 \\
\hline TK 6-A3b & 1.50 & 1.37 & 1.46 & $\mathbf{1 . 4 4}$ & 0.06 \\
\hline TK 6-C3a & 1.14 & 1.15 & 1.12 & $\mathbf{1 . 1 4}$ & 0.02 \\
\hline Tk 8 simulant sludge & 1.34 & 1.47 & 1.41 & $\mathbf{1 . 4 1}$ & 0.07 \\
\hline
\end{tabular}

Table 4 Tank 6F Composite Sample Bulk Density, g/mL

\begin{tabular}{|l|c|c|c|c|c|}
\hline Tank 6F Sample ID & Run-1 & Run-2 & Run-3 & Average & Stdev. \\
\hline Tk 6 Composite No. 1 & 1.33 & 1.31 & 1.32 & $\mathbf{1 . 3 2}$ & 0.01 \\
\hline Tk 6 Composite No. 2 & 1.33 & 1.26 & 1.28 & $\mathbf{1 . 2 9}$ & 0.04 \\
\hline Tk 6 Composite No. 3 & 1.41 & 1.38 & 1.36 & $\mathbf{1 . 3 9}$ & 0.02 \\
\hline Tank 8 Simulant sludge\# & 1.40 & 1.41 & 1.42 & $\mathbf{1 . 4 1}$ & 0.01 \\
\hline
\end{tabular}

\# Out of cell bulk density determination using the reference Tank 8 simulant sludge were within $5 \%$ of the cell values reported here for the Tank 8 simulant sludge. 
Table 5 Tank 6F Sample Compositions for Composite Samples 1, 2 and 3.

\begin{tabular}{|c|c|c|c|c|c|c|}
\hline $\begin{array}{l}\text { Composite } \\
\text { Sample \#s }\end{array}$ & $\begin{array}{c}\text { Material } \\
\text { Available, } g\end{array}$ & $\begin{array}{c}\text { Proportional } \\
\text { Sample } \\
\text { location } \\
\text { volume, \% } \\
\end{array}$ & $\begin{array}{c}\text { Homogenized } \\
\text { Bulk density, } \\
\text { g/mL }\end{array}$ & $\begin{array}{c}\text { Wt. } \\
\text { Fraction }\end{array}$ & $\begin{array}{c}\text { Required wt. } \\
\text { of material to } \\
\text { make } 70 \mathrm{~g} \\
\text { composite }\end{array}$ & $\begin{array}{c}\text { Amount } \\
\text { Weighed, g }\end{array}$ \\
\hline \multicolumn{7}{|c|}{ Comp. Sample \#1 } \\
\hline $6-\mathrm{A} 1 \mathrm{a}$ & 55.17 & 31 & 1.19 & 0.28 & 19.88 & 19.877 \\
\hline $6-\mathrm{A} 1 \mathrm{~b}$ & 78.94 & 31 & 1.55 & 0.37 & 25.89 & 25.894 \\
\hline 6-B1a & 49.55 & 11.5 & 1.34 & 0.12 & 8.31 & 8.305 \\
\hline 6-B1b & 82.12 & 11.5 & 1.16 & 0.10 & 7.19 & 7.187 \\
\hline 6-C1a & 44.85 & 15 & 1.08 & 0.12 & 8.73 & 8.728 \\
\hline Mass sum, $\mathrm{g}$ & 310.63 & NA & NA & NA & 70.00 & 69.991 \\
\hline \multicolumn{7}{|c|}{ Comp. Sample \#2 } \\
\hline $6-\mathrm{A} 2 \mathrm{a}$ & 117.26 & 27 & 1.44 & 0.30 & 20.68 & 20.684 \\
\hline $6-\mathrm{A} 2 \mathrm{~b}$ & 83.47 & 27 & 1.34 & 0.27 & 19.24 & 19.242 \\
\hline $6-\mathrm{B} 2 \mathrm{a}$ & 87.14 & 13 & 1.26 & 0.12 & 8.71 & 8.715 \\
\hline $6-\mathrm{B} 2 \mathrm{~b}$ & 81.09 & 13 & 1.32 & 0.13 & 9.13 & 9.132 \\
\hline $6-\mathrm{C} 2 \mathrm{a}$ & 43.68 & 20 & 1.15 & 0.17 & 12.23 & 12.228 \\
\hline Mass sum, $\mathbf{g}$ & 412.64 & NA & NA & NA & 70.00 & 70.001 \\
\hline \multicolumn{7}{|c|}{ Comp. Sample \#3 } \\
\hline $6-\mathrm{A} 3 \mathrm{a}$ & 109.06 & 25 & 1.32 & 0.25 & 17.64 & 17.636 \\
\hline $6-\mathrm{A} 3 \mathrm{~b}$ & 99.37 & 25 & 1.44 & 0.27 & 19.24 & 19.244 \\
\hline $6-\mathrm{B} 3 \mathrm{a}$ & NA & NA & NA & NA & NA & NA \\
\hline $6-\mathrm{B} 3 \mathrm{~b}$ & 51.94 & 31 & 1.30 & 0.31 & 21.54 & 21.546 \\
\hline 6-C3a & 86.26 & 19 & 1.14 & 0.17 & 11.58 & 11.578 \\
\hline Mass sum, $\mathrm{g}$ & 346.63 & NA & NA & NA & 70.00 & 70.004 \\
\hline
\end{tabular}

NA Sample not available. See reference-2.

Table 6 Weight Percent Solids for Tank 6F Composite Samples, Wt\%

\begin{tabular}{|l|c|c|c|c|c|}
\hline Tank 6F Sample ID & Run-1 & Run-2 & Run-3 & Average & Stdev. \\
\hline Tk 6 Composite No. 1 & 87.8 & 87.4 & 87.8 & $\mathbf{8 7 . 7}$ & 0.23 \\
\hline Tk 6 Composite No. 2 & 89.3 & 89.7 & 89.9 & $\mathbf{8 9 . 6}$ & 0.31 \\
\hline Tk 6 Composite No. 3 & 91.3 & 91.3 & 91.1 & $\mathbf{9 1 . 2}$ & 0.13 \\
\hline & & & & & 0.14 \\
\hline Tank 8 Simulant sludge & 91.2 & 91.2 & 91.0 & $\mathbf{9 1 . 1}$ & 0.10 \\
\hline $\begin{array}{l}\text { *5\% Reference NaCl } \\
\text { Salt solution }\end{array}$ & 4.9 & 4.7 & 4.8 & 4.8 & \\
\hline
\end{tabular}

$*$ Reference target $\mathrm{wt} \% \mathrm{NaCl}$ solid $=4.9 \%$ 


\subsection{Blank Evaluations and Reference Materials}

Two types of reference matrices were used during the characterization of Tank $6 \mathrm{~F}$ samples. The first reference material was an analyzed reference glass (ARG) which was stored outside the shielded cells but processed in the shielded cells along with the samples during sample preparations. The second was a dried Tank 8 simulant sludge, which was exposed to the shielded cell radiological environment in which the Tank $6 \mathrm{~F}$ radionuclide material was processed prior to analysis. The elemental chemical composition of the Tank 8 simulant sludge and analyzed reference glass are presented in Appendices A-3 and A-4. Distilled and de-ionized water was used as the liquid reagent media and blanks in all cell digestion cases.

The absence of radionuclides in these reference materials allowed the materials to additionally be utilized as blanks for radiochemical analyses.

Prior to the processing of the Tank $6 \mathrm{~F}$ samples, which normally involved the opening of selected samples to be blended together, two in-cell reference Tank 8 simulant sludge samples in $250-\mathrm{mL}$ capacity poly-bottles were placed at strategic locations in the shielded cell to ensure that these reference samples were exposed to the same cell environments as the Tank $6 \mathrm{~F}$ samples. Each simulant sludge reference sample container held about 20 grams of Tank 8 simulant sludge. The containers were opened when the Tank $6 \mathrm{~F}$ samples were being processed or air dried and closed at the end of each day of work in the cell. At the end of each Tank $6 \mathrm{~F}$ sample preparations or digestion (aqua regia and peroxide fusion digestions), the Tank 8 simulant sludge reference material was also prepared in a manner similar to that for the preparation of Tank $6 \mathrm{~F}$ samples and submitted for the same analyses as the actual samples from Tank 6F.

\subsection{Leaching Characterization of Tank $6 \mathrm{~F}$ Solids}

Known quantities of homogenized Tank 6F composite solids were leached with distilled and deionized water and analyzed in triplicate. An average of $1.24 \pm 0.04$ grams of the composite solids was leached with an average of $50.01 \pm 0.01$ grams of distilled and de-ionized water. In this process each solid fraction was thoroughly mixed with the given amount of distilled and deionized water, and the mixture was hand agitated (shielded cell manipulator) for a total of about five minutes and left to stand for another 24 hours before another agitation and filtering of the mixture using a 0.45 micron Nalgene filter unit. The filtrate from the mixture was analyzed in triplicate for anion components as required. Thus, only surface-bound and water soluble constituents are assumed to be accounted for in the leachate analyses.

\subsection{RESULTS}

Appendix A (Appendices A-1 and A-2) contains the SRNL Analytical Development Laboratory Information Management System (LIMS) numbers for tracking the analytical data presented in this report. Details of most of the analytical methodologies including weight percent solids and density determinations applied in Tank 6F sample characterizations are summarized in Appendix B. It is worth pointing out that many digestion methods were performed in the shielded cells prior to taking representative sample aliquots out of the cells for analyses. Additionally, many of the initial separations for challenging radionuclide characterizations were performed in the Shielded Cells. 
In the Tank $6 \mathrm{~F}$ composite sample characterization results presented below, values preceded by "<" (less than sign) indicate values were below minimum detection limits, and values proceeded by " $\leq$ " (less than or equal to sign) indicate that for replicates, at least one of the analysis values was above the instrument or method detection limit. Thus, where replicate analyses were both above and below the detection limit, the average of all replicates above and below the detection limit is given and a " $\leq$ " sign precedes the average value. The standard deviation values were calculated only for values that were above the detection limits. The minimum detectable activity (MDA) is defined as the value above which instrument signal can be considered real and the upper limit (UL) is defined as activity observed but biased high due to spectral interference or blank contamination. The detection limit (DL) as used in mass spectrometer or ICP-ES analyses is equivalent to three times the standard deviation of the blank measurements.

The one sigma percent uncertainty for each major radionuclide, as reported in the tables, is based on the pooled estimate derived from the individual uncertainties for each replicate measurement for that radionuclide $\left[\operatorname{SQRT}\left(\left(\operatorname{SUMSQ}\left(\mathrm{x}_{\mathrm{i}}\right) / \mathrm{n}\right)\right)\right]$, where $\mathrm{n}$ is the number of replicates and $\mathrm{x}_{\mathrm{i}}$ is the individual uncertainty associated with each radionuclide for each run. Here it is assumed that the radio-analytical processes, be it counting or other techniques, are of the same precision for each individual measurement.

Occasionally, situations were encountered where the samples prepared and analyzed in triplicate gave mixed results with one or two of the triplicate analyses results being less than the MDA. In these cases, the reporting of the one sigma percent uncertainty is presented in a slightly different format. In this situation, the individual percent uncertainty associated with each run for that radionuclide is reported along with MDA or upper limit values as indicated by the analytical method. For example, under the one sigma percent uncertainty column for the isotope Np-239 in Table 14, the 21.7/MDA designation implies that the one sigma percent uncertainty for Np-239 in run 1 is reported with values above the detection limit and thus has a one sigma percent uncertainty of 21.7 percent. The measurements (runs 2 and 3) for $\mathrm{Np}-239$ which were below the detection limit and thus not assigned percent uncertainty values are assigned an MDA. Similarly, in the analysis result for Eu-155 (runs 2 and 3, Table 13), the pooled statistics for the one sigma percent uncertainty is 5 and since the third run result of $<2.17 \mathrm{E}+00 \mathrm{uCi} / \mathrm{g}$ was due to spectral interference the result is only reported as an upper limit. Thus, the one sigma percent uncertainty for that set of runs for Eu-155 is presented as 5/UL.

To monitor potential sample contamination during processing, analytical blank (reagent blanks and Tank 8 simulant sludge) results were compared to sample analytical results (See second column blank results for most radionuclides as presented in each Table). Although analyses of the ARG and Tank 8 simulant solids blanks both provided valid measures of potential radionuclide contamination, results for the Tank 8 simulant solids blank were judged more appropriate for two primary reasons: 1) the Tank 8 simulant solids aliquots were carried through the entire series of Shielded Cells preparation and digestion steps, just like the tank samples (while the ARG aliquots were prepared outside of the Shielded Cells and then only digested in the Shielded Cells); and 2) the dilution factors for the Tank 8 simulant solids aliquots were consistent with those of the tank samples (while the dilution factors for the ARG aliquots were approximately four times those of the tank samples). For these reasons, all blank values reported for radionuclide analyses were based on Tank 8 simulant data (instead of the ARG data). 
It is worth noting that when reporting results in units of concentration (as opposed to straight activity), the impact of increased dilution is inflation in the apparent magnitude of detected contamination. For example, in the case of Pu-238 (where blank contamination was detected), the Pu-238 activity measured in the Tank 8 simulant blank was $7.3-9.7 \%$ of the Pu- 238 measured in the samples, and the Pu-238 activity measured in the ARG blank was $5.4-7.1 \%$ of the Pu-238 measured in the samples. These levels of blank contamination were all deemed acceptable, based on the measurement performance criterion requiring that blank activity be less than $10 \%$ of the sample activity. In contrast, in terms of concentrations (activity per mass), the $\mathrm{Pu}-238$ concentration in the Tank 8 simulant blank was 7.6 - 9.7\% of the Pu-238 concentrations in the samples, while the Pu-238 concentration in the ARG blank was $23-29 \%$ of the Pu-238 concentrations in the samples. Clearly, the apparent inflation of contaminant concentrations for the ARG matrix is an artifact of adjusting the ARG data for $4 \mathrm{x}$ greater dilution than the sample data. Thus, taking all these into consideration the conclusion is that there were no significant cross contamination issues either from the environment of the shielded cell staging and operation areas or the reagents used in sample preparations.

The reporting units for all radionuclides including $\mathrm{PF}$ and $\mathrm{AQR}$ digestion analytical results are presented per gram of composite Tank $6 \mathrm{~F}$ sample. Correction for water content as determined by sub-sample drying at $110{ }^{\circ} \mathrm{C}$, if required (original "as received" basis to dry basis), can be accomplished through the use of the dry solid weight percent (wt \%) values as shown in Table 6 for each composite sample. For example, $\mu \mathrm{Ci} / \mathrm{g}$ dried solids $=[\mathrm{x} \mu \mathrm{Ci} / \mathrm{g}$ of "as-received solids * (100 $\mathrm{g}$ of "as-received solids) $/ 87.7 \mathrm{~g}$ dried solids]; using composite sample 1 in Table 6 . Here $\mathrm{x}$ $\mu \mathrm{Ci} / \mathrm{g}$ represents the unknown activity of the "as-received" solids.

The one sigma analytical measurement uncertainty value for all of the anions and transition metals reported here is 20 percent. Leaching results are presented per gram of the "homogenized and composite" Tank $6 \mathrm{~F}$ composite samples.

Tables 7 through 9 show the water soluble anion constituents for the Tank $6 \mathrm{~F}$ composite sample, while Tables 10 through 12 contain inorganic constituent analytical results for the three composite Tank $6 \mathrm{~F}$ samples. Tables 13 through 15 show the analytical results for the standard radiological constituents for the three composite Tank $6 \mathrm{~F}$ samples. The following color codes are used for the Table contents: Green for blank values, red for less than values, pink for less than or equal to values, and bold for averages. All subsequent tables have similar color code meanings.

\subsection{Data Quality and Presentations for Routine Radionuclide Constituents}

The ICP-MS results are given for each atomic mass and in most cases each mass number represents only one isotope. An example of an exception is mass 238, since both uranium and plutonium are represented by this mass number. However, since the mass contribution of U-238 is significantly greater than that of $\mathrm{Pu}-238$, the 238 signal is used to quantify U-238, not Pu-238. For this reason, Pu-238 was determined by PUTTA (chemical separation coupled with alpha spectroscopy). See Appendix B for summaries of the methods. In cases where ICP-MS and 
radiochemistry data give similar results for a species, radiochemistry is typically selected due to better sensitivity and precision.

While many of the minimum detection limits (MDL), as specified in the TTR and TTQAP were met for the species characterized for Tank $6 \mathrm{~F}$ composite samples, some were not met. In a number of cases, the relatively high levels of radioactive species of the same element or a chemically similar element precluded the ability to measure an isotope to lower levels. For example, the high activities of americium and curium isotopes in the sample matrix raised the instrumental backgrounds for $\mathrm{Cm}-243$, having a detrimental effect on the detection limit for that isotope (Appendix A; Tables A5-1 through Tables A5-3). The 2.6 year half-life Pm-147 coextracts with the 90 year half-life Sm-151. Both have overlapping beta spectra, with slightly higher continuum beta end-point energy for Pm-147. The Sm-151 levels in these composite Tank 6F samples were relatively high, substantially raising the detection limit achievable for Pm147. A number of gamma emitting radionuclides were analyzed using a Cs-137 removed gamma analysis. Cesium-137 was expected to be the main contributor to background levels which would decrease the sensitivity of the gamma analysis for other species. While that was true, the samples also contained significant quantities of other gamma emitting isotopes (i.e. Co-60) which raised the background and thus the detection limits for other gamma emitting species. The minimum detection limits for Eu-152, Cm-243 and Cf-249 were not met because of spectral interferences.

Data reported for Tank 6F composite samples on Am-241 and Am-243 are based on analytical result from Cs-removed gamma analyzes. However, a second set of confirmatory analytical results for Am-241 and Am-243 based on Am/Cm analytical methods which are used mainly to validate the first set of Cs-removed gamma data is also presented in Appendix A, Table A5-1. This second set of data for Am-241 and Am-243 overlaps the first set of data and are within 10\% of the Cs-removed data.

While reviewing the data tables, one of the triplicate $\mathrm{Cm}-242$ values was observed to be roughly an order of magnitude lower in concentration then the results of the other eight Cm-242 measured values in the other two triplicate groups as well as the remaining 2 values of the sample's triplicate group. The discrepancy led to a further review of the sample's $\mathrm{Am} / \mathrm{Cm}$ alpha spectrum fit. The alpha spectrum was dominated by Cm-244, Am-241 and Am-243. The alpha peak corresponding to $\mathrm{Cm}-242$ had $\sim 0.1 \%$ of the alpha counts measured in the spectrum. The low statistics of the counts in the Cm-242 region of interest led to a poor spectral peak shape which proved to be challenging to the spectroscopy software peak fitting algorithm. The Cm242 peak fitting region of interest had to be adjusted several times manually to finally provide a satisfactory fit to the $\mathrm{Cm}-242$ region of interest. The resulting fit provided a Cm-242 value which fell right in line with the other 8 Tank $6 \mathrm{Cm}-242$ values.

\subsection{Data Quality and Presentations for Elemental Constituents}

The reference, non-radioactive materials, used for the elemental analyses results presented in Tables 10 through 12 were a reference glass standard, ARG and dried Tank 8 simulant sludge samples. Appendices A-3 and A-4 contain the elemental analytical results for the two reference materials in comparison to their known reference values. 3,4 
A comparison of the laboratory results for the cations present in the simulant sludge, with sodium being the exception, shows that the laboratory analytical results are not significantly different from the known reference values for these cations. The percent relative deviation (\%RD defined as [difference/mean] $\left.{ }^{*} 100\right)$ for 12 of the 13 constituent cations of this simulant sludge material averaged less than $6 \%$. Only sodium analysis reference result had a \%RD greater than $20 \%$. Similarly, looking at the analytical results for the 18 elemental constituents of the ARG reference sample [Appendix A-4], the average percent relative deviation for all the constituents is less than $3 \%$.

The value for sodium in the Tank 8 simulant sludge has not been completely quantified because sodium is likely more in the soluble phase and we were primarily interested in the solid phase of the simulant (simulant was settled, fluid decanted and wet solid cake dried to obtain only the solid fraction). Thus, the higher difference in sodium is not unexpected. Overall, the laboratory analytical results from these two reference materials for the constituent elements are quite comparable to the expected or known concentrations.

Analytical elemental results were also compared between different methods used for characterization of Tank $6 \mathrm{~F}$ composite samples, specifically comparing results from inductively coupled plasma-mass spectrometer (ICP-MS) with results from inductively coupled plasmaemission spectroscopy (ICP-ES). The concentration of select cations (natural lanthanum, barium and lead) were calculated from ICP-MS information and the resulting concentration values compared with the ICP-ES corresponding results presented in this report. Typical calculations are shown in Appendix A-5 for $\mathrm{La}, \mathrm{Ba}$ and $\mathrm{Pb}$. The average percent relative deviation between ICP-MS and ICP-ES analytical results for $\mathrm{La}, \mathrm{Ba}$ and $\mathrm{Pb}$ were between 3 and 15\%. These comparison results are summarized in Appendix A-5 and show that ICP-ES analytical results are about the same order of magnitude as the ICP-MS data for these select cations.

Because of the low iodine concentration in the Tank $6 \mathrm{~F}$ leachate samples, analyses for iodine by mass spectroscopy was preferred over analyses by IC. Leached Tank $6 \mathrm{~F}$ composite sample analyses for iodine by mass spectroscopy for stable iodine, assuming 100\% iodine natural abundance, was based on the assumption that all other elements with mass 127 (Xe-127, Sn-127, Cs-127, Ba-127, La-127, In-127 etc.) have relatively short half-lives ranging from milliseconds to a few days. Thus, the total stable iodine reported in Tables 7 through 9 for elemental iodine is based on mass spectroscopy data for mass-127. The sum of iodine in each Tank 6F composite sample is approximated by adding mass 127 stable iodine results with mass 129 radioactive iodine data.

\subsection{Data Quality and Presentations for Non-Routine Radionuclide Analytes.}

Some of the radionuclides are not present in easily measurable concentrations and in some cases there was significant sample matrix effect as in the cases of carbon-14 (C-14) analyses in the Tank $6 \mathrm{~F}$ composite samples. Thus, existing standard methods are not sufficient in attaining the requested minimum detection limits. These cases required new method development to meet the low detection limit requirements and minimize spectral interferences. Isotopes measured for the Tank 6F composite samples which fall into this category of analyses also include Zr-93, Ac-227, Th-229/230, Pa-231, Ra-226, Nb-94, Sn-126, Sb-126 and Sb-126m. With the exception of the 
antimony and tin isotopes (Sn-126, Sb-126 and Sb-126m), most of the targeted minimum detection limits for these radionuclides were met.

Thorium-229/230 analyses blanks for all three composite Tank 6F samples showed "no- yields" for Th-229/Th-230. A no-yield implies that there was no activity observed in the sample. Only Tank 6F composite sample number 3, run 1 showed no measurable yield for Th-229. Tank 6F composite sample number 3, run 1 result for Ac-227 showed a poor yield and thus no data is presented.

Difficulties encountered during the C-14 analyses in the Shielded Cells required repeated sets of sample preparations and re-engineered carbon isolation vessels to produce data of acceptable quality. The first set of Tank $6 \mathrm{~F} \mathrm{C}-14$ determinations was performed using the same analytical method and labware successfully utilized in the Tank 5F C-14 analyses. Using this approach, sample aliquots were digested and processed in a manner that liberated C-14 in the form of gaseous carbon dioxide, and then captured the carbon dioxide in an aqueous solution conducive to liquid scintillation counting. C-14 standards and blanks were processed and analyzed along with the sample aliquots, to provide a measure of the relative carbon recoveries and the absence or presence of $\mathrm{C}-14$ contamination. As in the case of the Tank 5F C-14 analyses, the first set of Tank 6F C-14 analyses utilized a series of "Mason jar-like" vessels to liberate and collect the carbon dioxide, prior to capturing it in a medium submitted to Analytical Development. In the Tank $5 \mathrm{~F}$ analyses, the liberation vessels and the seals used to prevent loss of carbon dioxide gas were all new (never used before). In contrast, in the Tank 6 analyses, the vessels and seals were those previously used for the Tank $5 \mathrm{~F}$ analyses, following cleaning and decontamination.

The first set of Tank $6 \mathrm{~F} \mathrm{C-14}$ analytical results indicated that the vessel seals were ineffective, as evidenced by carbon dioxide losses and $\mathrm{C}-14$ contamination of blanks. As a consequence, the first set of C-14 results was rejected. A second set of Tank 6F C-14 analyses was performed after cleaning the existing vessels and replacing the seals. Unfortunately, the results of the second set of Tank $6 \mathrm{~F}$ analyses suffered one of the same problems as the first set of Tank $6 \mathrm{~F}$ analyses -- carbon dioxide losses. Hence, the second set of C-14 results was also rejected.

Because of the problems encountered with the "Mason jar-like" vessels, new collection vessels generated from Erlenmeyer flasks were fabricated at SRNL glass shop. Utilizing the new vessels, the $\mathrm{C}-14$ analyses were repeated once again for a new set of Tank $6 \mathrm{~F}$ sample aliquots. This time, the results were considerably more consistent, with both effective carbon dioxide capture and insignificant blank contamination. Although the recoveries for the C-14 spiked simulant were slightly outside of the targeted range (67-133\% versus the $75-125 \%$ target), the results were deemed acceptable, given the high level of resources that would have been needed to hone the method further. The reported results include this larger method uncertainty in the pooled calculations.

Composite Tank 6F samples contained significant quantities of other gamma emitting isotopes (i.e. Co-60, Eu-154, Eu-155, etc.), which raised the spectral background. Because of this raised background, the detection limits for other gamma emitting species with lower activities were not met (i.e. Sb-126, Sn-126, and Sb-126m). 
Table 7 Weight Percent Anions Leached per gram of Tank 6F- Composite Sample \#1

\begin{tabular}{|c|c|c|c|c|c|c|}
\hline Anion & Run-1 & Run-2 & Run-3 & Average & Std. Dev. & Unit \\
\hline Fluoride, $\mathrm{F}^{1-}$ & $<0.04$ & $<0.04$ & $<0.04$ & $<\mathbf{0 . 0 4}$ & & $\mathrm{Wt} \%$ \\
\hline Formate, $\mathrm{CHO}_{2}{ }^{1-}$ & $<0.04$ & $<0.04$ & $<0.04$ & $<\mathbf{0 . 0 4}$ & & $\mathrm{Wt} \%$ \\
\hline Chloride, $\mathrm{Cl}^{1-}$ & $<0.04$ & $<0.04$ & $<0.04$ & $<\mathbf{0 . 0 4}$ & & $\mathrm{Wt} \%$ \\
\hline Nitrite, $\mathrm{NO}_{2}{ }^{1-}$ & $3.07 \mathrm{E}+00$ & $3.18 \mathrm{E}+00$ & $3.19 \mathrm{E}+00$ & $\mathbf{3 . 1 5}+00$ & $7.0 \mathrm{E}-02$ & $\mathrm{Wt} \%$ \\
\hline Bromide, $\mathrm{Br}^{1-}$ & $<0.19$ & $<0.22$ & $<0.21$ & $<0.21$ & & $\mathrm{Wt} \%$ \\
\hline Nitrate, $\mathrm{NO}_{3}{ }^{1-}$ & $3.23 \mathrm{E}+00$ & $3.34 \mathrm{E}+00$ & $3.35 \mathrm{E}+00$ & $\mathbf{3 . 3 1 E}+\mathbf{0 0}$ & $7.0 \mathrm{E}-02$ & $\mathrm{Wt} \%$ \\
\hline Phosphate, $\mathrm{PO}_{4}{ }^{3-}$ & $<0.04$ & $<0.04$ & $<0.04$ & $<\mathbf{0 . 0 4}$ & & $\mathrm{Wt} \%$ \\
\hline Sulfate, $\mathrm{SO}_{4}{ }^{2-}$ & $1.03 \mathrm{E}+00$ & $1.07 \mathrm{E}+00$ & $1.01 \mathrm{E}+00$ & $\mathbf{1 . 0 3 E}+\mathbf{0 0}$ & $3.0 \mathrm{E}-02$ & $\mathrm{Wt} \%$ \\
\hline Oxalate, $\mathrm{C}_{2} \mathrm{O}_{4}{ }^{2-}$ & $1.16 \mathrm{E}+00$ & $1.13 \mathrm{E}+00$ & $1.25 \mathrm{E}+00$ & $\mathbf{1 . 1 8 E}+\mathbf{0 0}$ & $6.0 \mathrm{E}-02$ & $\mathrm{Wt} \%$ \\
\hline Iodine, $\mathrm{I}-127$ & $1.39 \mathrm{E}-04$ & $1.19 \mathrm{E}-04$ & $1.39 \mathrm{E}-04$ & $\mathbf{1 . 3 2 E}-\mathbf{0 4}$ & $1.20 \mathrm{E}-05$ & $\mathrm{Wt} \%$ \\
\hline Iodine, $\mathrm{I}-129$ & $7.88 \mathrm{E}-05$ & $6.69 \mathrm{E}-05$ & $8.16 \mathrm{E}-05$ & $\mathbf{7 . 5 7 E - 0 5}$ & $7.82 \mathrm{E}-06$ & $\mathrm{Wt} \%$ \\
\hline Total Iodine & $2.18 \mathrm{E}-04$ & $1.86 \mathrm{E}-04$ & $2.21 \mathrm{E}-04$ & $\mathbf{2 . 0 8 E - 0 4}$ & $1.93 \mathrm{E}-05$ & $\mathrm{Wt} \%$ \\
\hline
\end{tabular}

Table 8 Weight Percent Anions Leached per gram of Tank 6F- Composite Sample \#2

\begin{tabular}{|c|c|c|c|c|c|c|}
\hline Anion & Run-1 & Run-2 & Run-3 & Average & Std. Dev. & Unit \\
\hline Fluoride, $\mathrm{F}^{1-}$ & $<0.04$ & $<0.04$ & $<0.04$ & $<\mathbf{0 . 0 4}$ & & $\mathrm{Wt} \%$ \\
\hline Formate, $\mathrm{CHO}_{2}{ }^{1-}$ & $<0.04$ & $<0.04$ & $<0.04$ & $<\mathbf{0 . 0 4}$ & & $\mathrm{Wt} \%$ \\
\hline Chloride, $\mathrm{Cl}^{1-}$ & $<0.04$ & $<0.04$ & $<0.04$ & $<\mathbf{0 . 0 4}$ & & $\mathrm{Wt} \%$ \\
\hline Nitrite, $\mathrm{NO}_{2}{ }^{1-}$ & $3.08 \mathrm{E}+00$ & $3.08 \mathrm{E}+00$ & $3.09 \mathrm{E}+00$ & $\mathbf{3 . 0 8 E}+\mathbf{0 0}$ & $1.0 \mathrm{E}-02$ & $\mathrm{Wt} \%$ \\
\hline Bromide, $\mathrm{Br}^{1-}$ & $<0.19$ & $<0.20$ & $<0.20$ & $<\mathbf{0 . 2 0}$ & & $\mathrm{Wt} \%$ \\
\hline Nitrate, $\mathrm{NO}_{3}{ }^{1-}$ & $3.24 \mathrm{E}+00$ & $3.27 \mathrm{E}+00$ & $3.24 \mathrm{E}+00$ & $\mathbf{3 . 2 5 E}+\mathbf{0 0}$ & $2.0 \mathrm{E}-02$ & $\mathrm{Wt} \%$ \\
\hline Phosphate, $\mathrm{PO}_{4}{ }^{3-}$ & $<0.04$ & $<0.04$ & $<0.04$ & $<\mathbf{0 . 0 4}$ & & $\mathrm{Wt} \%$ \\
\hline Sulfate, $\mathrm{SO}_{4}{ }^{2-}$ & $1.03 \mathrm{E}+00$ & $9.90 \mathrm{E}-01$ & $1.04 \mathrm{E}+00$ & $\mathbf{1 . 0 2 E}+\mathbf{0 0}$ & $3.0 \mathrm{E}-02$ & $\mathrm{Wt} \%$ \\
\hline Oxalate, $\mathrm{C}_{2} \mathrm{O}_{4}{ }^{2-}$ & $1.00 \mathrm{E}+00$ & $1.18 \mathrm{E}+00$ & $1.16 \mathrm{E}+00$ & $\mathbf{1 . 1 1 E}+\mathbf{0 0}$ & $1.0 \mathrm{E}-01$ & $\mathrm{Wt} \%$ \\
\hline Iodine, $\mathrm{I}-127$ & $1.47 \mathrm{E}-04$ & $3.51 \mathrm{E}-04$ & $2.99 \mathrm{E}-04$ & $\mathbf{2 . 6 6 E - 0 4}$ & $1.06 \mathrm{E}-04$ & $\mathrm{Wt} \%$ \\
\hline Iodine, $\mathrm{I}-129$ & $9.12 \mathrm{E}-05$ & $9.35 \mathrm{E}-05$ & $8.22 \mathrm{E}-05$ & $\mathbf{8 . 9 0 E}-05$ & $6.00 \mathrm{E}-06$ & $\mathrm{Wt} \%$ \\
\hline Total Iodine & $2.38 \mathrm{E}-04$ & $4.44 \mathrm{E}-04$ & $3.81 \mathrm{E}-04$ & $\mathbf{3 . 5 5 E - 0 4}$ & $1.06 \mathrm{E}-04$ & $\mathrm{Wt} \%$ \\
\hline
\end{tabular}

Table 9 Weight Percent Anions Leached per gram of Tank 6F- Composite Sample \#3

\begin{tabular}{|c|c|c|c|c|c|c|}
\hline Anion & Run-1 & Run-2 & Run-3 & Average & Std. Dev. & Unit \\
\hline Fluoride, $\mathrm{F}^{1-}$ & $<0.04$ & $<0.04$ & $<0.04$ & $<\mathbf{0 . 0 4}$ & & $\mathrm{Wt} \%$ \\
\hline Formate, $\mathrm{CHO}_{2}{ }^{1-}$ & $<0.04$ & $<0.04$ & $<0.04$ & $<\mathbf{0 . 0 4}$ & & $\mathrm{Wt} \%$ \\
\hline Chloride, $\mathrm{Cl}^{1-}$ & $<0.04$ & $<0.04$ & $<0.04$ & $<\mathbf{0 . 0 4}$ & & $\mathrm{Wt} \%$ \\
\hline Nitrite, $\mathrm{NO}_{2}{ }^{-1}$ & $3.23 \mathrm{E}+00$ & $3.32 \mathrm{E}+00$ & $3.28 \mathrm{E}+00$ & $\mathbf{3 . 2 8 E}+\mathbf{0 0}$ & $4.0 \mathrm{E}-02$ & $\mathrm{Wt} \%$ \\
\hline Bromide, $\mathrm{Br}^{1-}$ & $<0.20$ & $<0.21$ & $<0.20$ & $<\mathbf{0 . 2 0}$ & & $\mathrm{Wt} \%$ \\
\hline Nitrate, $\mathrm{NO}_{3}{ }^{1-}$ & $3.21 \mathrm{E}+00$ & $3.41 \mathrm{E}+00$ & $3.32 \mathrm{E}+00$ & $\mathbf{3 . 3 1 E}+\mathbf{0 0}$ & $1.0 \mathrm{E}-01$ & $\mathrm{Wt} \%$ \\
\hline Phosphate, $\mathrm{PO}_{4}{ }^{3-}$ & $<0.04$ & $<0.04$ & $<0.04$ & $<\mathbf{0 . 0 4}$ & & $\mathrm{Wt} \%$ \\
\hline Sulfate, $\mathrm{SO}_{4}{ }^{--}$ & $1.01 \mathrm{E}+00$ & $1.07 \mathrm{E}+00$ & $1.01 \mathrm{E}+00$ & $\mathbf{1 . 0 3 E}+\mathbf{0 0}$ & $4.0 \mathrm{E}-01$ & $\mathrm{Wt} \%$ \\
\hline Oxalate, $\mathrm{C}_{2} \mathrm{O}_{4}{ }^{2-}$ & $1.42 \mathrm{E}+00$ & $1.27 \mathrm{E}+00$ & $1.40 \mathrm{E}+00$ & $\mathbf{1 . 3 6 E}+\mathbf{0 0}$ & $8.0 \mathrm{E}-02$ & $\mathrm{Wt} \%$ \\
\hline Iodine, $\mathrm{I}-127$ & $3.78 \mathrm{E}-04$ & $4.37 \mathrm{E}-04$ & $2.52 \mathrm{E}-04$ & $\mathbf{3 . 5 6 E - 0 4}$ & $9.46 \mathrm{E}-05$ & $\mathrm{Wt} \%$ \\
\hline Iodine, $\mathrm{I}-129$ & $1.04 \mathrm{E}-04$ & $1.08 \mathrm{E}-04$ & $1.07 \mathrm{E}-04$ & $\mathbf{1 . 0 6 E}-04$ & $2.29 \mathrm{E}-06$ & $\mathrm{Wt} \%$ \\
\hline Total Iodine & $4.82 \mathrm{E}-04$ & $5.45 \mathrm{E}-04$ & $3.59 \mathrm{E}-04$ & $\mathbf{4 . 6 2 E}-04$ & $9.49 \mathrm{E}-05$ & $\mathrm{Wt} \%$ \\
\hline
\end{tabular}


SRNL-STI-2012-00365, Rev. 0

Page 22 of 94

Table 10 Elemental Constituents in Tank 6F Composite Sample \# 1, wt\%

\begin{tabular}{|c|c|c|c|c|c|}
\hline Analytes & Tank 6F, Run1, wt\% & Tank 6F, Run 2, wt\% & Tank 6F, Run 3, wt\% & Average, wt $\%$ & STDEV \\
\hline Ag & $<1.12 \mathrm{E}-02$ & $<1.13 \mathrm{E}-02$ & $<1.13 \mathrm{E}-02$ & $<1.13 \mathrm{E}-02$ & \\
\hline Al & $2.22 \mathrm{E}+00$ & $2.20 \mathrm{E}+00$ & $2.24 \mathrm{E}+00$ & $2.22 \mathrm{E}+00$ & $2.00 E-02$ \\
\hline As & $<5.37 \mathrm{E}-04$ & $<5.41 \mathrm{E}-04$ & $<5.40 \mathrm{E}-04$ & $<5.39 \mathrm{E}-04$ & \\
\hline B & $<1.58 \mathrm{E}-02$ & $<1.59 \mathrm{E}-02$ & $<1.59 \mathrm{E}-02$ & $<1.59 \mathrm{E}-02$ & \\
\hline $\mathbf{B a}$ & $1.11 \mathrm{E}-01$ & $1.10 \mathrm{E}-01$ & $1.05 \mathrm{E}-01$ & 1.09E-01 & $3.21 E-03$ \\
\hline $\mathrm{Be}$ & $<4.69 \mathrm{E}-04$ & $<4.72 \mathrm{E}-04$ & $<4.72 \mathrm{E}-04$ & $<4.71 \mathrm{E}-04$ & \\
\hline $\mathbf{C a}$ & $3.58 \mathrm{E}-01$ & 3.62E-01 & $3.64 \mathrm{E}-01$ & 3.61E-01 & $3.06 E-03$ \\
\hline Cd & $1.03 \mathrm{E}-02$ & $9.87 \mathrm{E}-03$ & $9.90 \mathrm{E}-03$ & 1.00E-02 & $2.40 E-04$ \\
\hline $\mathrm{Ce}$ & $1.81 \mathrm{E}-01$ & $1.81 \mathrm{E}-01$ & $1.73 \mathrm{E}-01$ & $1.78 \mathrm{E}-01$ & $4.62 E-03$ \\
\hline Co & $2.13 \mathrm{E}-02$ & 1.99E-02 & $2.02 \mathrm{E}-02$ & 2.05E-02 & 7.37E-04 \\
\hline $\mathrm{Cr}$ & $5.05 \mathrm{E}-02$ & 4.64E-02 & 4.71E-02 & 4.80E-02 & 2.19E-03 \\
\hline $\mathrm{Cu}$ & $1.02 \mathrm{E}-01$ & $9.65 \mathrm{E}-02$ & $9.44 \mathrm{E}-02$ & 9.76E-02 & $3.92 E-03$ \\
\hline $\mathbf{F e}$ & $2.03 \mathrm{E}+01$ & $1.93 \mathrm{E}+01$ & $1.83 \mathrm{E}+01$ & $1.93 \mathrm{E}+01$ & $1.00 E+00$ \\
\hline Gd & $<1.68 \mathrm{E}-02$ & $<1.69 \mathrm{E}-02$ & $<1.69 \mathrm{E}-02$ & $<1.69 \mathrm{E}-02$ & \\
\hline Hg & 4.19E-01 & $3.63 \mathrm{E}-01$ & $3.51 \mathrm{E}-01$ & $3.78 \mathrm{E}-01$ & 0.036 \\
\hline $\mathbf{K}$ & $7.21 \mathrm{E}-02$ & $6.53 \mathrm{E}-02$ & $6.43 \mathrm{E}-02$ & 6.72E-02 & 4.24E-03 \\
\hline $\mathbf{L a}$ & $8.76 \mathrm{E}-02$ & $8.42 \mathrm{E}-02$ & $8.10 \mathrm{E}-02$ & 8.43E-02 & $3.30 E-03$ \\
\hline $\mathbf{L i}$ & $1.04 \mathrm{E}-01$ & $9.98 \mathrm{E}-02$ & $9.39 \mathrm{E}-02$ & 9.92E-02 & 5.07E-03 \\
\hline Mg & 4.89E-02 & $4.88 \mathrm{E}-02$ & 4.75E-02 & 4.84E-02 & 7.81E-04 \\
\hline Mn & $1.55 \mathrm{E}+00$ & $1.56 \mathrm{E}+00$ & $1.51 \mathrm{E}+00$ & $1.54 \mathrm{E}+00$ & $2.65 E-02$ \\
\hline Mo & $6.45 \mathrm{E}-03$ & $6.61 \mathrm{E}-03$ & $6.83 \mathrm{E}-03$ & $6.63 \mathrm{E}-03$ & $1.91 E-04$ \\
\hline $\mathbf{N a}$ & $1.32 \mathrm{E}+01$ & $1.35 \mathrm{E}+01$ & $1.36 \mathrm{E}+01$ & $1.34 \mathrm{E}+01$ & 2.08E-01 \\
\hline $\mathbf{N i}$ & $4.60 \mathrm{E}+00$ & $4.45 \mathrm{E}+00$ & $4.33 \mathrm{E}+00$ & $4.46 \mathrm{E}+00$ & $1.35 E-01$ \\
\hline $\mathbf{P}$ & $3.22 \mathrm{E}-02$ & $2.59 \mathrm{E}-02$ & $1.41 \mathrm{E}-02$ & 2.41E-02 & 9.19E-03 \\
\hline $\mathbf{P b}$ & $4.98 \mathrm{E}-02$ & 4.91E-02 & 4.73E-02 & 4.87E-02 & 1.29E-03 \\
\hline $\mathbf{S}$ & $3.75 \mathrm{E}-01$ & $4.04 \mathrm{E}-01$ & 4.30E-01 & 4.03E-01 & $2.75 E-02$ \\
\hline Sb & $<9.98 \mathrm{E}-02$ & $<1.01 \mathrm{E}-01$ & $<1.00 \mathrm{E}-01$ & $<1.00 \mathrm{E}-01$ & \\
\hline $\mathrm{Se}$ & $<1.07 \mathrm{E}-03$ & $<1.08 \mathrm{E}-03$ & $<1.08 \mathrm{E}-03$ & $<1.08 \mathrm{E}-03$ & \\
\hline $\mathbf{S i}$ & $6.13 \mathrm{E}-01$ & $5.97 \mathrm{E}-01$ & $6.00 \mathrm{E}-01$ & 6.03E-01 & 8.50E-03 \\
\hline Sn & $5.09 \mathrm{E}-03$ & $6.04 \mathrm{E}-03$ & $4.51 \mathrm{E}-03$ & $5.21 \mathrm{E}-03$ & $7.72 E-04$ \\
\hline $\mathrm{Sr}$ & 4.39E-02 & $4.69 \mathrm{E}-02$ & $4.53 \mathrm{E}-02$ & $4.54 \mathrm{E}-02$ & $1.50 E-03$ \\
\hline Th & $<1.25 \mathrm{E}-01$ & $<1.26 \mathrm{E}-01$ & $<1.26 \mathrm{E}-01$ & $<1.26 \mathrm{E}-01$ & \\
\hline $\mathbf{T i}$ & $1.89 \mathrm{E}-02$ & $1.83 \mathrm{E}-02$ & $1.76 \mathrm{E}-02$ & $1.83 \mathrm{E}-02$ & $6.51 E-04$ \\
\hline $\mathbf{U}$ & $3.31 \mathrm{E}+00$ & $3.55 \mathrm{E}+00$ & $3.45 \mathrm{E}+00$ & $3.44 \mathrm{E}+00$ & $1.21 E-01$ \\
\hline $\mathbf{V}$ & $<3.67 \mathrm{E}-03$ & $<3.70 \mathrm{E}-03$ & $<3.69 \mathrm{E}-03$ & $<3.69 \mathrm{E}-03$ & \\
\hline Zn & $1.02 \mathrm{E}-01$ & $9.59 \mathrm{E}-02$ & $9.28 \mathrm{E}-02$ & 9.69E-02 & $4.68 E-03$ \\
\hline $\mathbf{Z r}$ & $1.56 \mathrm{E}-01$ & $1.66 \mathrm{E}-01$ & $1.20 \mathrm{E}-01$ & $1.47 \mathrm{E}-01$ & $2.42 E-02$ \\
\hline
\end{tabular}


SRNL-STI-2012-00365, Rev. 0

Page 23 of 94

Table 11 Elemental Constituents in Tank 6F Composite Sample \# 2, wt\%

\begin{tabular}{|c|c|c|c|c|c|}
\hline Analytes & Tank 6F, Run1, wt\% & Tank 6F, Run 2, wt\% & Tank 6F, Run 3, wt\% & Average, wt $\%$ & STDEV \\
\hline $\mathrm{Ag}$ & $<1.15 \mathrm{E}-02$ & $<1.12 \mathrm{E}-02$ & $<1.15 \mathrm{E}-02$ & $<1.14 \mathrm{E}-02$ & \\
\hline Al & $2.29 \mathrm{E}+00$ & $2.35 \mathrm{E}+00$ & $2.34 \mathrm{E}+00$ & $2.33 \mathrm{E}+00$ & $3.21 E-02$ \\
\hline As & $<5.49 \mathrm{E}-04$ & $<5.33 \mathrm{E}-04$ & $<5.48 \mathrm{E}-04$ & $<5.43 \mathrm{E}-04$ & \\
\hline B & $<1.61 \mathrm{E}-02$ & $<1.57 \mathrm{E}-02$ & $<1.61 \mathrm{E}-02$ & $<1.60 \mathrm{E}-02$ & \\
\hline $\mathbf{B a}$ & $1.14 \mathrm{E}-01$ & $1.16 \mathrm{E}-01$ & $1.16 \mathrm{E}-01$ & $1.15 \mathrm{E}-01$ & $1.15 E-03$ \\
\hline $\mathrm{Be}$ & $<4.79 \mathrm{E}-04$ & $<4.65 \mathrm{E}-04$ & $<4.78 \mathrm{E}-04$ & $<4.74 \mathrm{E}-04$ & \\
\hline $\mathbf{C a}$ & 4.24E-01 & $4.16 \mathrm{E}-01$ & $4.29 \mathrm{E}-01$ & $4.23 \mathrm{E}-01$ & $6.56 E-03$ \\
\hline Cd & $1.04 \mathrm{E}-02$ & $1.07 \mathrm{E}-02$ & $1.08 \mathrm{E}-02$ & $1.06 \mathrm{E}-02$ & $2.08 E-04$ \\
\hline $\mathrm{Ce}$ & $1.85 \mathrm{E}-01$ & $1.88 \mathrm{E}-01$ & $1.88 \mathrm{E}-01$ & 1.87E-01 & $1.73 E-03$ \\
\hline Co & $2.24 \mathrm{E}-02$ & $2.23 \mathrm{E}-02$ & $2.26 \mathrm{E}-02$ & 2.24E-02 & $1.53 E-04$ \\
\hline $\mathrm{Cr}$ & $4.75 \mathrm{E}-02$ & $4.86 \mathrm{E}-02$ & 4.77E-02 & 4.79E-02 & 5.86E-04 \\
\hline $\mathrm{Cu}$ & $1.01 \mathrm{E}-01$ & $1.01 \mathrm{E}-01$ & $1.01 \mathrm{E}-01$ & $1.01 \mathrm{E}-01$ & 0.00 \\
\hline $\mathrm{Fe}$ & $2.05 \mathrm{E}+01$ & $2.03 \mathrm{E}+01$ & $2.05 \mathrm{E}+01$ & $2.04 \mathrm{E}+01$ & $1.15 E-01$ \\
\hline Gd & $<1.72 \mathrm{E}-02$ & $<1.67 \mathrm{E}-02$ & $<1.71 \mathrm{E}-02$ & $<1.70 \mathrm{E}-02$ & \\
\hline $\mathrm{Hg}$ & $3.74 \mathrm{E}-01$ & $3.69 \mathrm{E}-01$ & $3.68 \mathrm{E}-01$ & $3.70 \mathrm{E}-01$ & $3.00 E-03$ \\
\hline $\mathbf{K}$ & $7.50 \mathrm{E}-02$ & $6.77 \mathrm{E}-02$ & $7.21 \mathrm{E}-02$ & $7.16 \mathrm{E}-02$ & $3.68 E-03$ \\
\hline $\mathbf{L a}$ & $8.88 \mathrm{E}-02$ & $8.97 \mathrm{E}-02$ & $9.01 \mathrm{E}-02$ & 8.95E-02 & $6.66 E-04$ \\
\hline $\mathbf{L i}$ & $1.03 \mathrm{E}-01$ & $9.98 \mathrm{E}-02$ & $1.02 \mathrm{E}-01$ & 1.02E-01 & $1.64 E-03$ \\
\hline Mg & $5.16 \mathrm{E}-02$ & $5.18 \mathrm{E}-02$ & $5.24 \mathrm{E}-02$ & 5.19E-02 & 4.16E-04 \\
\hline Mn & $1.66 \mathrm{E}+00$ & $1.68 \mathrm{E}+00$ & $1.73 \mathrm{E}+00$ & $1.69 \mathrm{E}+00$ & $3.61 E-02$ \\
\hline Mo & $6.72 \mathrm{E}-03$ & $6.68 \mathrm{E}-03$ & $6.69 \mathrm{E}-03$ & $6.70 \mathrm{E}-03$ & $2.08 E-05$ \\
\hline $\mathbf{N a}$ & $1.22 \mathrm{E}+01$ & $1.29 \mathrm{E}+01$ & $1.25 \mathrm{E}+01$ & $1.25 \mathrm{E}+01$ & $3.51 E-01$ \\
\hline $\mathbf{N i}$ & $4.67 \mathrm{E}+00$ & $4.65 \mathrm{E}+00$ & $4.72 \mathrm{E}+00$ & $4.68 \mathrm{E}+00$ & $3.61 E-02$ \\
\hline $\mathbf{P}$ & $3.73 \mathrm{E}-02$ & $3.87 \mathrm{E}-02$ & $2.64 \mathrm{E}-02$ & $3.41 \mathrm{E}-02$ & $6.73 E-03$ \\
\hline $\mathbf{P b}$ & $5.16 \mathrm{E}-02$ & $4.98 \mathrm{E}-02$ & $5.08 \mathrm{E}-02$ & 5.07E-02 & $9.02 E-04$ \\
\hline $\mathbf{S}$ & $3.53 \mathrm{E}-01$ & $4.08 \mathrm{E}-01$ & $3.76 \mathrm{E}-01$ & $3.79 \mathrm{E}-01$ & $2.76 E-02$ \\
\hline $\mathrm{Sb}$ & $<1.02 \mathrm{E}-01$ & $<9.90 \mathrm{E}-02$ & $<1.02 \mathrm{E}-01$ & $<1.01 \mathrm{E}-01$ & \\
\hline $\mathrm{Se}$ & $<1.10 \mathrm{E}-03$ & $<1.07 \mathrm{E}-03$ & $<1.10 \mathrm{E}-03$ & $<1.09 \mathrm{E}-03$ & \\
\hline $\mathbf{S i}$ & $6.37 \mathrm{E}-01$ & $6.43 \mathrm{E}-01$ & $5.98 \mathrm{E}-01$ & $6.26 \mathrm{E}-01$ & $2.44 E-02$ \\
\hline Sn & $5.13 \mathrm{E}-03$ & $6.50 \mathrm{E}-03$ & $5.43 \mathrm{E}-03$ & $5.69 \mathrm{E}-03$ & $7.20 \mathrm{E}-04$ \\
\hline $\mathrm{Sr}$ & $5.00 \mathrm{E}-02$ & $5.03 \mathrm{E}-02$ & $5.13 \mathrm{E}-02$ & 5.05E-02 & $6.81 E-04$ \\
\hline Th & $<1.28 \mathrm{E}-01$ & $<1.24 \mathrm{E}-01$ & $<1.28 \mathrm{E}-01$ & $<1.27 \mathrm{E}-01$ & \\
\hline $\mathbf{T i}$ & $1.89 \mathrm{E}-02$ & $1.92 \mathrm{E}-02$ & $1.92 \mathrm{E}-02$ & $1.91 \mathrm{E}-02$ & $1.73 E-04$ \\
\hline $\mathbf{U}$ & $3.65 \mathrm{E}+00$ & $3.58 \mathrm{E}+00$ & $3.82 \mathrm{E}+00$ & $3.68 \mathrm{E}+00$ & $1.23 E-01$ \\
\hline $\mathbf{V}$ & $<3.75 \mathrm{E}-03$ & $<3.64 \mathrm{E}-03$ & $<3.74 \mathrm{E}-03$ & $<3.71 \mathrm{E}-03$ & \\
\hline $\mathrm{Zn}$ & $1.00 \mathrm{E}-01$ & $1.00 \mathrm{E}-01$ & $9.88 \mathrm{E}-02$ & $9.96 \mathrm{E}-02$ & $6.93 E-04$ \\
\hline $\mathbf{Z r}$ & $2.43 \mathrm{E}-01$ & $2.07 \mathrm{E}-01$ & $2.25 \mathrm{E}-01$ & 2.25E-01 & $1.80 E-02$ \\
\hline
\end{tabular}


SRNL-STI-2012-00365, Rev. 0

Page 24 of 94

Table 12 Elemental Constituents in Tank 6F Composite Sample \# 3, wt\%

\begin{tabular}{|c|c|c|c|c|c|}
\hline Analytes & Tank 6F, Run1, wt\% & Tank 6F, Run 2, wt\% & Tank 6F, Run 3, wt\% & Average, wt $\%$ & STDEV \\
\hline Ag & $<1.14 \mathrm{E}-02$ & $<1.15 \mathrm{E}-02$ & $<1.12 \mathrm{E}-02$ & $<1.14 \mathrm{E}-02$ & \\
\hline Al & $2.38 \mathrm{E}+00$ & $2.41 \mathrm{E}+00$ & $2.43 \mathrm{E}+00$ & $2.41 \mathrm{E}+00$ & $2.52 E-02$ \\
\hline As & $<5.43 \mathrm{E}-04$ & $<5.48 \mathrm{E}-04$ & $<5.36 \mathrm{E}-04$ & $<5.42 \mathrm{E}-04$ & \\
\hline B & $<1.60 \mathrm{E}-02$ & $<1.61 \mathrm{E}-02$ & $<1.58 \mathrm{E}-02$ & $<1.60 \mathrm{E}-02$ & \\
\hline Ba & $1.26 \mathrm{E}-01$ & $1.31 \mathrm{E}-01$ & $1.30 \mathrm{E}-01$ & $1.29 \mathrm{E}-01$ & $2.65 E-03$ \\
\hline $\mathrm{Be}$ & $<4.74 \mathrm{E}-04$ & $<4.78 \mathrm{E}-04$ & $<4.68 \mathrm{E}-04$ & $<4.73 \mathrm{E}-04$ & \\
\hline $\mathbf{C a}$ & $3.64 \mathrm{E}-01$ & $3.77 \mathrm{E}-01$ & $3.87 \mathrm{E}-01$ & $3.76 \mathrm{E}-01$ & $1.15 E-02$ \\
\hline Cd & $1.08 \mathrm{E}-02$ & $1.09 \mathrm{E}-02$ & $1.13 \mathrm{E}-02$ & 1.10E-02 & $2.65 E-04$ \\
\hline $\mathrm{Ce}$ & $2.22 \mathrm{E}-01$ & $2.30 \mathrm{E}-01$ & $2.29 \mathrm{E}-01$ & 2.27E-01 & 4.36E-03 \\
\hline Co & $2.63 \mathrm{E}-02$ & $2.65 \mathrm{E}-02$ & $2.73 \mathrm{E}-02$ & 2.67E-02 & $5.29 E-04$ \\
\hline $\mathrm{Cr}$ & $5.26 \mathrm{E}-02$ & 5.39E-02 & $5.42 \mathrm{E}-02$ & $5.36 \mathrm{E}-02$ & 8.50E-04 \\
\hline $\mathbf{C u}$ & $1.10 \mathrm{E}-01$ & $1.10 \mathrm{E}-01$ & $1.08 \mathrm{E}-01$ & 1.09E-01 & $1.15 E-03$ \\
\hline $\mathrm{Fe}$ & $2.11 \mathrm{E}+01$ & $2.10 \mathrm{E}+01$ & $2.17 \mathrm{E}+01$ & $2.13 E+01$ & $3.79 E-01$ \\
\hline Gd & $<1.70 \mathrm{E}-02$ & $<1.71 \mathrm{E}-02$ & $<1.68 \mathrm{E}-02$ & $<1.70 \mathrm{E}-02$ & \\
\hline $\mathrm{Hg}$ & $3.97 \mathrm{E}-01$ & 4.04E-01 & $4.02 \mathrm{E}-01$ & 4.01E-01 & 4.00E-03 \\
\hline $\mathbf{K}$ & $6.47 \mathrm{E}-02$ & $6.61 \mathrm{E}-02$ & $6.68 \mathrm{E}-02$ & $6.59 \mathrm{E}-02$ & $1.07 E-03$ \\
\hline La & $1.03 \mathrm{E}-01$ & $1.07 \mathrm{E}-01$ & $1.07 \mathrm{E}-01$ & 1.06E-01 & $2.31 E-03$ \\
\hline $\mathbf{L i}$ & $1.08 \mathrm{E}-01$ & $1.05 \mathrm{E}-01$ & $1.14 \mathrm{E}-01$ & 1.09E-01 & $4.58 E-03$ \\
\hline Mg & $5.67 \mathrm{E}-02$ & $5.75 \mathrm{E}-02$ & $5.88 \mathrm{E}-02$ & 5.77E-02 & $1.06 E-03$ \\
\hline Mn & $1.82 \mathrm{E}+00$ & $1.99 \mathrm{E}+00$ & $1.96 \mathrm{E}+00$ & $1.92 \mathrm{E}+00$ & 9.07E-02 \\
\hline Mo & $6.63 \mathrm{E}-03$ & $7.55 \mathrm{E}-03$ & $7.38 \mathrm{E}-03$ & $7.19 \mathrm{E}-03$ & $4.90 E-04$ \\
\hline $\mathrm{Na}$ & $1.15 \mathrm{E}+01$ & $1.13 \mathrm{E}+01$ & $1.13 \mathrm{E}+01$ & $1.14 \mathrm{E}+01$ & $1.15 E-01$ \\
\hline $\mathbf{N i}$ & $5.35 \mathrm{E}+00$ & $5.45 \mathrm{E}+00$ & $5.51 \mathrm{E}+00$ & $5.44 \mathrm{E}+00$ & $8.08 E-02$ \\
\hline $\mathbf{P}$ & $1.79 \mathrm{E}-02$ & $3.03 \mathrm{E}-02$ & $2.41 \mathrm{E}-02$ & 2.41E-02 & $6.20 E-03$ \\
\hline $\mathbf{P b}$ & $5.46 \mathrm{E}-02$ & $5.22 \mathrm{E}-02$ & $5.46 \mathrm{E}-02$ & 5.38E-02 & 1.39E-03 \\
\hline $\mathbf{S}$ & $3.97 \mathrm{E}-01$ & $3.73 \mathrm{E}-01$ & $3.96 \mathrm{E}-01$ & 3.89E-01 & $1.36 E-02$ \\
\hline $\mathrm{Sb}$ & $<1.01 \mathrm{E}-01$ & $<1.02 \mathrm{E}-01$ & $<9.96 \mathrm{E}-02$ & $<1.01 \mathrm{E}-01$ & \\
\hline $\mathrm{Se}$ & $<1.09 \mathrm{E}-03$ & $<1.10 \mathrm{E}-03$ & $<1.07 \mathrm{E}-03$ & $<1.09 \mathrm{E}-03$ & \\
\hline $\mathbf{S i}$ & $6.84 \mathrm{E}-01$ & $6.85 \mathrm{E}-01$ & $6.72 \mathrm{E}-01$ & $6.80 \mathrm{E}-01$ & 7.23E-03 \\
\hline Sn & $6.14 \mathrm{E}-03$ & $5.36 \mathrm{E}-03$ & $5.23 \mathrm{E}-03$ & $5.58 \mathrm{E}-03$ & 4.92E-04 \\
\hline $\mathrm{Sr}$ & $5.12 \mathrm{E}-02$ & $5.29 \mathrm{E}-02$ & $5.40 \mathrm{E}-02$ & $5.27 \mathrm{E}-02$ & $1.41 E-03$ \\
\hline Th & $<1.27 \mathrm{E}-01$ & $<1.28 \mathrm{E}-01$ & $<1.25 \mathrm{E}-01$ & $<1.27 \mathrm{E}-01$ & \\
\hline Ti & $2.09 \mathrm{E}-02$ & $2.07 \mathrm{E}-02$ & $2.15 \mathrm{E}-02$ & $2.10 \mathrm{E}-02$ & 4.16E-04 \\
\hline $\mathbf{U}$ & $4.42 \mathrm{E}+00$ & $4.54 \mathrm{E}+00$ & $4.49 \mathrm{E}+00$ & $4.48 \mathrm{E}+00$ & $6.03 E-02$ \\
\hline $\mathbf{V}$ & $<3.72 \mathrm{E}-03$ & $<3.74 \mathrm{E}-03$ & $<3.66 \mathrm{E}-03$ & $<3.71 \mathrm{E}-03$ & \\
\hline $\mathrm{Zn}$ & $1.08 \mathrm{E}-01$ & $1.03 \mathrm{E}-01$ & $1.11 \mathrm{E}-01$ & 1.07E-01 & $4.04 E-03$ \\
\hline $\mathbf{Z r}$ & $2.19 \mathrm{E}-01$ & $1.68 \mathrm{E}-01$ & $1.52 \mathrm{E}-01$ & $1.80 \mathrm{E}-01$ & $3.50 E-02$ \\
\hline
\end{tabular}


SRNL-STI-2012-00365, Rev. 0

Page 25 of 94

Table 13 Radiological Constituents for Tank 6F Composite Sample \#1, $\mu \mathrm{Ci} / \mathrm{g}$.

\begin{tabular}{|c|c|c|c|c|c|c|c|c|}
\hline Analytes* & Blank & Run 1 & Run 2 & Run 3 & Average & Stdev & \%Uncert. & $\begin{array}{c}\text { Targeted } \\
\text { Minimum } \\
\text { Detection } \\
\text { Limits } \\
\end{array}$ \\
\hline Gross alpha & $<1.92 \mathrm{E}-02$ & $<2.10 \mathrm{E}+02$ & $<2.26 \mathrm{E}+02$ & $<2.00 \mathrm{E}+02$ & $<2.12 \mathrm{E}+02$ & & MDA & None \\
\hline $\begin{array}{c}\text { Non-volatile } \\
\text { beta }\end{array}$ & $6.88 \mathrm{E}-01$ & $3.94 \mathrm{E}+04$ & $3.76 \mathrm{E}+04$ & $3.70 \mathrm{E}+04$ & $3.80 \mathrm{E}+04$ & $1.24 E+03$ & 10.0 & None \\
\hline H-3 & $<2.06 \mathrm{E}-02$ & $<2.01 \mathrm{E}-02$ & $<2.03 \mathrm{E}-02$ & $<2.03 \mathrm{E}-02$ & $<2.02 \mathrm{E}-02$ & & MDA & $1.0 \mathrm{E}-01$ \\
\hline $\mathrm{C}-14$ & $<7.34 \mathrm{E}-04$ & $2.22 \mathrm{E}-02$ & $1.22 \mathrm{E}-02$ & $2.13 \mathrm{E}-02$ & $1.86 \mathrm{E}-02$ & $5.56 \mathrm{E}-03$ & 48.7 & $1.0 \mathrm{E}-01$ \\
\hline Ni-59 & $<3.91 \mathrm{E}-02$ & $5.32 \mathrm{E}+00$ & $2.71 \mathrm{E}+00$ & $3.59 \mathrm{E}+00$ & $3.87 \mathrm{E}+00$ & $1.32 E+00$ & 10.0 & $9.0 \mathrm{E}-02$ \\
\hline Ni-63 & 2.04E-01 & $2.83 \mathrm{E}+02$ & $1.64 \mathrm{E}+02$ & $3.64 \mathrm{E}+02$ & $2.70 \mathrm{E}+02$ & $1.00 E+02$ & 10.0 & $1.0 \mathrm{E}-01$ \\
\hline Co-60 & $<1.02 \mathrm{E}-02$ & $7.03 \mathrm{E}+00$ & $7.12 \mathrm{E}+00$ & $7.07 \mathrm{E}+00$ & $7.07 \mathrm{E}+00$ & $4.50 E-02$ & 5.0 & $1.0 \mathrm{E}-03$ \\
\hline Se-79 & $<2.80 \mathrm{E}-04$ & $1.15 \mathrm{E}-02$ & $1.59 \mathrm{E}-02$ & $1.50 \mathrm{E}-02$ & 1.41E-02 & $2.31 E-03$ & 32.2 & $1.0 \mathrm{E}-03$ \\
\hline Sr-90 & $<6.58 \mathrm{E}+00$ & $1.57 \mathrm{E}+04$ & $1.61 \mathrm{E}+04$ & $1.54 \mathrm{E}+04$ & $1.57 E+04$ & $3.83 E+02$ & 6.7 & $1.0 \mathrm{E}-03$ \\
\hline $\mathrm{Y}-90$ & $<6.58 \mathrm{E}+00$ & $1.57 \mathrm{E}+04$ & $1.61 \mathrm{E}+04$ & $1.54 \mathrm{E}+04$ & $1.57 E+04$ & $3.83 E+02$ & 6.7 & $1.0 \mathrm{E}-03$ \\
\hline Zr-93 & $6.10 \mathrm{E}-03$ & $8.40 \mathrm{E}-01$ & $7.87 \mathrm{E}-01$ & $6.26 \mathrm{E}-01$ & 7.51E-01 & $1.11 E-01$ & 20 & $1.0 \mathrm{E}-03$ \\
\hline $\mathrm{Nb}-94$ & $<2.90 \mathrm{E}-04$ & $<1.74 \mathrm{E}-03$ & $<1.72 \mathrm{E}-03$ & $<2.04 \mathrm{E}-03$ & $<1.83 \mathrm{E}-03$ & & MDA & $3.0 \mathrm{E}-03$ \\
\hline Tc-99 & $<1.45 \mathrm{E}-04$ & $1.24 \mathrm{E}-01$ & $1.18 \mathrm{E}-01$ & $1.07 \mathrm{E}-01$ & $1.17 \mathrm{E}-01$ & 8.50E-03 & 6.8 & $1.0 \mathrm{E}-03$ \\
\hline Sn-126 & $<2.46 \mathrm{E}-02$ & $<7.30 \mathrm{E}-01$ & $<7.30 \mathrm{E}-01$ & $<7.30 \mathrm{E}-01$ & $<7.30 \mathrm{E}-01$ & & MDA & $1.0 \mathrm{E}-03$ \\
\hline $\mathrm{Sb}-126$ & $<9.91 \mathrm{E}-03$ & $<9.41 \mathrm{E}-02$ & $<9.50 \mathrm{E}-02$ & $<9.41 \mathrm{E}-02$ & $<9.44 \mathrm{E}-02$ & & MDA & $1.0 \mathrm{E}-03$ \\
\hline Sb-126m & $<9.91 \mathrm{E}-03$ & $<9.41 \mathrm{E}-02$ & $<9.50 \mathrm{E}-02$ & $<9.41 \mathrm{E}-02$ & $<9.44 \mathrm{E}-02$ & & MDA & $1.0 \mathrm{E}-03$ \\
\hline I-129 & $<5.27 \mathrm{E}-06$ & $1.39 \mathrm{E}-04$ & $1.18 \mathrm{E}-04$ & $1.44 \mathrm{E}-04$ & $1.33 E-04$ & 1.39E-05 & 7.5 & $1.0 \mathrm{E}-04$ \\
\hline Cs-135 & $<2.27 \mathrm{E}-05$ & $2.64 \mathrm{E}-03$ & $2.77 \mathrm{E}-03$ & $3.04 \mathrm{E}-03$ & 2.82E-03 & $2.04 \mathrm{E}-04$ & 20 & $5.0 \mathrm{E}-02$ \\
\hline Cs-137 & $<2.74 \mathrm{E}-02$ & $5.36 \mathrm{E}+02$ & $5.32 \mathrm{E}+02$ & $5.36 \mathrm{E}+02$ & $5.35 \mathrm{E}+02$ & $2.60 E+00$ & 5.0 & $1.0 \mathrm{E}-03$ \\
\hline $\mathrm{Ba}-137 \mathrm{~m}$ & $<2.59 \mathrm{E}-02$ & $5.07 \mathrm{E}+02$ & $5.03 \mathrm{E}+02$ & $5.07 \mathrm{E}+02$ & $5.06 \mathrm{E}+02$ & $2.46 E+00$ & 5.0 & $1.0 \mathrm{E}-03$ \\
\hline Pm-147 & $<2.40 \mathrm{E}-02$ & $<1.58 \mathrm{E}+02$ & $<1.49 \mathrm{E}+02$ & $<1.63 \mathrm{E}+02$ & $<1.56 \mathrm{E}+02$ & & UL & None \\
\hline Sm-151 & $<3.07 \mathrm{E}-02$ & $2.01 \mathrm{E}+02$ & $2.05 \mathrm{E}+02$ & $2.08 \mathrm{E}+02$ & $2.05 E+02$ & $3.40 E+00$ & 16.9 & $3.0 \mathrm{E}+00$ \\
\hline Eu-152 & $<2.73 \mathrm{E}-02$ & $<1.46 \mathrm{E}-01$ & $<1.47 \mathrm{E}-01$ & $<1.49 \mathrm{E}-01$ & $<1.47 \mathrm{E}-01$ & & MDA & $7.0 \mathrm{E}-03$ \\
\hline Eu-154 & $<1.90 \mathrm{E}-02$ & $1.60 \mathrm{E}+01$ & $1.59 \mathrm{E}+01$ & $1.59 \mathrm{E}+01$ & $1.59 \mathrm{E}+01$ & 4.50E-02 & 5 & $1.0 \mathrm{E}-03$ \\
\hline Eu-155 & $<2.80 \mathrm{E}-02$ & $3.16 \mathrm{E}+00$ & $<2.17 \mathrm{E}+00$ & $3.66 \mathrm{E}+00$ & $\leq 3.00 \mathrm{E}+00$ & & $5 / \mathrm{UL}$ & None \\
\hline $\mathrm{Ra}-226^{* * *}$ & No yield & $<2.65 \mathrm{E}-03$ & $<3.25 \mathrm{E}-03$ & $<2.76 \mathrm{E}-03$ & $<2.89 \mathrm{E}-03$ & & MDA & $5.0 \mathrm{E}-03$ \\
\hline Ac-227 & No yield & $<7.21 \mathrm{E}-06$ & $<1.50 \mathrm{E}-05$ & $<7.34 \mathrm{E}-06$ & $<9.83 \mathrm{E}-06$ & & $\mathrm{UL}$ & $1.30 \mathrm{E}-04$ \\
\hline Th-229 & No yield & $6.53 \mathrm{E}-05$ & 7.84E-05 & $5.59 \mathrm{E}-05$ & $6.65 \mathrm{E}-05$ & $1.13 \mathrm{E}-05$ & 18.1 & $1.0 \mathrm{E}-03$ \\
\hline Th-230 & No yield & $6.62 \mathrm{E}-04$ & $7.16 \mathrm{E}-04$ & 7.79E-04 & 7.19E-04 & $5.86 \mathrm{E}-05$ & 18.1 & $1.0 \mathrm{E}-03$ \\
\hline $\mathrm{Pa}-231$ & $<9.17 \mathrm{E}-04$ & $<4.08 \mathrm{E}-04$ & $<6.82 \mathrm{E}-04$ & $<8.15 \mathrm{E}-04$ & $<6.35 \mathrm{E}-04$ & & $\mathrm{DL}$ & $1.0 \mathrm{E}-03$ \\
\hline U-232 & No activity & $6.71 \mathrm{E}-05$ & $6.13 \mathrm{E}-05$ & $6.26 \mathrm{E}-05$ & 6.37E-05 & 3.07E-06 & 16.30 & $1.0 \mathrm{E}-03$ \\
\hline U-233 & $<1.21 \mathrm{E}-04$ & $2.86 \mathrm{E}-03$ & $2.86 \mathrm{E}-03$ & $3.57 \mathrm{E}-03$ & $3.10 \mathrm{E}-03$ & $4.10 E-04$ & 20.0 & $1.0 \mathrm{E}-03$ \\
\hline U-234 & $<7.81 \mathrm{E}-05$ & $7.77 \mathrm{E}-03$ & $7.81 \mathrm{E}-03$ & $9.69 \mathrm{E}-03$ & 8.42E-03 & $1.10 E-03$ & 20.0 & $1.0 \mathrm{E}-03$ \\
\hline U-235 & $<2.72 \mathrm{E}-08$ & $3.72 \mathrm{E}-04$ & $3.69 \mathrm{E}-04$ & $4.56 \mathrm{E}-04$ & 3.99E-04 & $4.94 E-05$ & 20.0 & $1.0 \mathrm{E}-04$ \\
\hline $\mathrm{U}-236$ & $<8.13 \mathrm{E}-07$ & $4.67 \mathrm{E}-04$ & $4.68 \mathrm{E}-04$ & $5.74 \mathrm{E}-04$ & $5.03 E-04$ & $6.15 E-05$ & 20.0 & $1.0 \mathrm{E}-03$ \\
\hline U-238 & $<8.17 \mathrm{E}-07$ & $1.35 \mathrm{E}-02$ & $1.28 \mathrm{E}-02$ & $1.33 \mathrm{E}-02$ & 1.32E-02 & $3.61 E-04$ & 20.0 & $1.0 \mathrm{E}-03$ \\
\hline Np-237 & $<1.48 \mathrm{E}-04$ & $1.50 \mathrm{E}-02$ & $1.11 \mathrm{E}-02$ & $1.49 \mathrm{E}-02$ & $1.37 \mathrm{E}-02$ & $2.22 E-03$ & 24.7 & $1.0 \mathrm{E}-03$ \\
\hline Pu-238 & $2.73 \mathrm{E}-01$ & $3.59 \mathrm{E}+00$ & $3.49 \mathrm{E}+00$ & $2.80 \mathrm{E}+00$ & $3.29 \mathrm{E}+00$ & $4.29 E-01$ & 9.9 & $1.0 \mathrm{E}-03$ \\
\hline Pu-239 & $3.34 \mathrm{E}-02$ & $2.89 \mathrm{E}+00$ & $2.99 \mathrm{E}+00$ & $2.95 \mathrm{E}+00$ & $2.94 \mathrm{E}+00$ & $5.03 E-02$ & 20 & $1.0 \mathrm{E}-03$ \\
\hline $\mathrm{Pu}-240$ & $<8.26 \mathrm{E}-03$ & $1.77 \mathrm{E}+00$ & $1.90 \mathrm{E}+00$ & $1.81 \mathrm{E}+00$ & $1.83 \mathrm{E}+00$ & $6.66 E-02$ & 20 & $1.0 \mathrm{E}-03$ \\
\hline $\mathrm{Pu}-239 / 240$ & $<1.08 \mathrm{E}-01$ & $4.64 \mathrm{E}+00$ & $4.91 \mathrm{E}+00$ & $4.77 \mathrm{E}+00$ & $4.77 E+00$ & $1.35 E-01$ & 8.2 & None \\
\hline $\mathrm{Pu}-241$ & $<9.50 \mathrm{E}-03$ & $7.12 \mathrm{E}+00$ & $6.53 \mathrm{E}+00$ & $6.17 \mathrm{E}+00$ & $6.61 \mathrm{E}+00$ & 4.77E-01 & 15.5 & $1.0 \mathrm{E}-03$ \\
\hline $\mathrm{Pu}-242$ & $<1.43 \mathrm{E}-04$ & $2.26 \mathrm{E}-03$ & $2.20 \mathrm{E}-03$ & $2.20 \mathrm{E}-03$ & 2.22E-03 & $3.46 E-05$ & 20 & $1.0 \mathrm{E}-03$ \\
\hline $\mathrm{Pu}-244$ & $<6.66 \mathrm{E}-07$ & $<7.89 \mathrm{E}-07$ & $<7.72 \mathrm{E}-07$ & $<6.62 \mathrm{E}-07$ & $<7.41 \mathrm{E}-07$ & & $\mathrm{DL}$ & $1.3 \mathrm{E}-04$ \\
\hline Am-241 & $<3.14 \mathrm{E}-02$ & $5.68 \mathrm{E}+01$ & $6.53 \mathrm{E}+01$ & $5.77 \mathrm{E}+01$ & $5.99 \mathrm{E}+01$ & $4.7 E+00$ & 5 & $1.0 \mathrm{E}-03$ \\
\hline Am-242m & $<1.36 \mathrm{E}-02$ & $1.00 \mathrm{E}-01$ & $1.10 \mathrm{E}-01$ & $1.09 \mathrm{E}-01$ & $1.06 \mathrm{E}-01$ & $5.63 E-03$ & 24.7 & $1.0 \mathrm{E}-03$ \\
\hline Np-239 & $<3.34 \mathrm{E}-02$ & $1.31 \mathrm{E}+00$ & $<2.25 \mathrm{E}+00$ & $1.15 \mathrm{E}+00$ & $\leq 1.57 \mathrm{E}+00$ & & 11.9/UL & None \\
\hline
\end{tabular}


SRNL-STI-2012-00365, Rev. 0

Page 26 of 94

Table 13 Continued. Radiological Constituents for Tank 6F Composite Sample \#1, $\mu \mathrm{Ci} / \mathrm{g}$.

\begin{tabular}{|c|c|c|c|c|c|c|c|c|}
\hline Am-243 & $<2.19 \mathrm{E}-02$ & $1.22 \mathrm{E}+00$ & $1.03 \mathrm{E}+00$ & $1.23 \mathrm{E}+00$ & $1.16 \mathrm{E}+00$ & $1.16 E-01$ & 13.3 & $1.0 \mathrm{E}-03$ \\
\hline $\mathrm{Cm}-242$ & $<1.13 \mathrm{E}-02$ & $8.24 \mathrm{E}-02$ & $9.14 \mathrm{E}-02$ & $9.01 \mathrm{E}-02$ & 8.80E-02 & $4.86 E-03$ & 24.71 & None \\
\hline Cm-243 & $<3.05 \mathrm{E}-03$ & $<5.18 \mathrm{E}-01$ & $<6.31 \mathrm{E}-01$ & $<8.87 \mathrm{E}-01$ & $<6.79 \mathrm{E}-01$ & & $U L$ & $2.0 \mathrm{E}-02$ \\
\hline $\mathrm{Cm}-244$ & $5.00 \mathrm{E}-01$ & $4.91 \mathrm{E}+01$ & $4.95 \mathrm{E}+01$ & $4.86 \mathrm{E}+01$ & $4.91 \mathrm{E}+01$ & $4.50 E-01$ & 15.10 & None \\
\hline $\mathrm{Cm}-245$ & $<1.27 \mathrm{E}-04$ & $4.82 \mathrm{E}-03$ & $4.91 \mathrm{E}-03$ & $4.73 \mathrm{E}-03$ & 4.82E-03 & $9.01 E-05$ & 20.0 & $2.0 \mathrm{E}-02$ \\
\hline $\mathrm{Cm}-246$ & $<6.35 \mathrm{E}-04$ & $3.20 \mathrm{E}-02$ & $3.30 \mathrm{E}-02$ & $3.36 \mathrm{E}-02$ & $3.29 \mathrm{E}-02$ & $7.97 E-04$ & 20.00 & None \\
\hline Cm-247 & $<5.23 \mathrm{E}-08$ & $<1.62 \mathrm{E}-07$ & $<2.05 \mathrm{E}-07$ & $<2.36 \mathrm{E}-07$ & $<2.01 \mathrm{E}-07$ & & $U L$ & $1.3 \mathrm{E}-04$ \\
\hline Bk-247 & $<5.86 \mathrm{E}-04$ & $<1.81 \mathrm{E}-03$ & $<2.28 \mathrm{E}-03$ & $<2.63 \mathrm{E}-03$ & $<2.24 \mathrm{E}-03$ & & UL & None \\
\hline Cm-248 & $<9.23 \mathrm{E}-06$ & $<1.49 \mathrm{E}-05$ & $<1.36 \mathrm{E}-05$ & $<1.26 \mathrm{E}-05$ & $<1.37 \mathrm{E}-05$ & & $U L$ & $1.3 \mathrm{E}-04$ \\
\hline Cf-249 & $<7.84 \mathrm{E}-04$ & $<5.27 \mathrm{E}-03$ & $<4.95 \mathrm{E}-03$ & $<5.90 \mathrm{E}-03$ & $<5.38 \mathrm{E}-03$ & & MDA & $5.0 \mathrm{E}-03$ \\
\hline Cf-250 & $<7.97 \mathrm{E}-05$ & $<3.82 \mathrm{E}-05$ & $<3.10 \mathrm{E}-05$ & $<6.35 \mathrm{E}-05$ & $<4.42 \mathrm{E}-05$ & & UL & None \\
\hline Cf-251 & $<1.73 \mathrm{E}-03$ & $<1.41 \mathrm{E}-02$ & $<1.32 \mathrm{E}-02$ & $<1.56 \mathrm{E}-02$ & $<1.43 \mathrm{E}-02$ & & MDA & None \\
\hline Cf-252 & $<1.13 \mathrm{E}-02$ & $<8.24 \mathrm{E}-02$ & $<9.14 \mathrm{E}-02$ & $<9.01 \mathrm{E}-02$ & $<8.80 \mathrm{E}-02$ & & UL & None \\
\hline
\end{tabular}

** While analysis of these isotopes is needed, meeting the detection limits for these isotopes is a lower priority than meeting detection limits for the other specified isotopes. 
SRNL-STI-2012-00365, Rev. 0

Page 27 of 94

Table 14 Radiological Constituents for Tank 6F Composite Sample \#2, $\mu \mathrm{Ci} / \mathrm{g}$.

\begin{tabular}{|c|c|c|c|c|c|c|c|c|}
\hline Analytes & Blank & Run 1 & Run 2 & Run 3 & Average & Stdev & \%Uncert. & $\begin{array}{c}\text { Targeted } \\
\text { Minimum } \\
\text { Detection } \\
\text { Limits } \\
\end{array}$ \\
\hline Gross alpha & $<1.92 \mathrm{E}-02$ & $<2.07 \mathrm{E}+02$ & $<2.13 \mathrm{E}+02$ & $<2.17 \mathrm{E}+02$ & $<2.12 \mathrm{E}+02$ & & MDA & None \\
\hline $\begin{array}{c}\text { Non-volatile } \\
\text { beta }\end{array}$ & $6.88 \mathrm{E}-01$ & $4.24 \mathrm{E}+04$ & $4.12 \mathrm{E}+04$ & $3.92 \mathrm{E}+04$ & $4.09 E+04$ & $1.59 E+03$ & 10.0 & None \\
\hline H-3 & $<2.06 \mathrm{E}-02$ & $<2.06 \mathrm{E}-02$ & $<2.00 \mathrm{E}-02$ & $<2.06 \mathrm{E}-02$ & $<2.04 \mathrm{E}-02$ & & MDA & $1.0 \mathrm{E}-01$ \\
\hline C-14 & $<7.34 \mathrm{E}-04$ & $1.55 \mathrm{E}-02$ & $2.36 \mathrm{E}-02$ & $2.38 \mathrm{E}-02$ & $2.10 \mathrm{E}-02$ & $4.73 \mathrm{E}-03$ & 48.6 & $1.0 \mathrm{E}-01$ \\
\hline $\mathrm{Ni}-59$ & $<3.91 \mathrm{E}-02$ & $3.98 \mathrm{E}+00$ & $4.59 \mathrm{E}+00$ & $3.32 \mathrm{E}+00$ & $3.96 \mathrm{E}+00$ & $6.40 E-01$ & 10.0 & $9.0 \mathrm{E}-02$ \\
\hline $\mathrm{Ni}-63$ & $2.04 \mathrm{E}-01$ & $3.03 \mathrm{E}+02$ & $2.54 \mathrm{E}+02$ & $3.09 \mathrm{E}+02$ & $2.88 \mathrm{E}+02$ & $3.02 E+01$ & 10.0 & $1.0 \mathrm{E}-01$ \\
\hline Co-60 & $<1.02 \mathrm{E}-02$ & $7.39 \mathrm{E}+00$ & $7.25 \mathrm{E}+00$ & $6.80 \mathrm{E}+00$ & $7.15 \mathrm{E}+00$ & $3.07 E-01$ & 5 & $1.0 \mathrm{E}-03$ \\
\hline Se-79 & $<2.80 \mathrm{E}-04$ & $3.40 \mathrm{E}-02$ & $1.37 \mathrm{E}-02$ & $1.63 \mathrm{E}-02$ & 2.13E-02 & $1.11 E-02$ & 47.6 & $1.0 \mathrm{E}-03$ \\
\hline Sr-90 & $<6.58 \mathrm{E}+00$ & $1.67 \mathrm{E}+04$ & $1.61 \mathrm{E}+04$ & $1.48 \mathrm{E}+04$ & $1.58 \mathrm{E}+04$ & $9.69 E+02$ & 6.7 & $1.0 \mathrm{E}-03$ \\
\hline Y-90 & $<6.58 \mathrm{E}+00$ & $1.67 \mathrm{E}+04$ & $1.61 \mathrm{E}+04$ & $1.48 \mathrm{E}+04$ & $1.58 \mathrm{E}+04$ & $9.69 E+02$ & 6.7 & $1.0 \mathrm{E}-03$ \\
\hline Zr-93 & $6.10 \mathrm{E}-03$ & $1.37 \mathrm{E}+00$ & $1.22 \mathrm{E}+00$ & $1.39 \mathrm{E}+00$ & $1.33 \mathrm{E}+00$ & $9.29 \mathrm{E}-02$ & 20 & $1.0 \mathrm{E}-03$ \\
\hline $\mathrm{Nb}-94$ & $<2.90 \mathrm{E}-04$ & $<1.86 \mathrm{E}-03$ & $<1.52 \mathrm{E}-03$ & $<1.01 \mathrm{E}-03$ & $<1.46 \mathrm{E}-03$ & & MDA & $3.0 \mathrm{E}-03$ \\
\hline Tc-99 & $<1.45 \mathrm{E}-04$ & $9.82 \mathrm{E}-02$ & $1.23 \mathrm{E}-01$ & $8.56 \mathrm{E}-02$ & $1.02 E-01$ & $1.93 E-02$ & 6.4 & $1.0 \mathrm{E}-03$ \\
\hline Sn-126 & $<2.46 \mathrm{E}-02$ & $<7.52 \mathrm{E}-01$ & $<6.40 \mathrm{E}-01$ & $<6.26 \mathrm{E}-01$ & $<6.73 \mathrm{E}-01$ & & MDA & $1.0 \mathrm{E}-03$ \\
\hline $\mathrm{Sb}-126$ & $<9.91 \mathrm{E}-03$ & $<9.64 \mathrm{E}-02$ & $<1.10 \mathrm{E}-01$ & $<1.08 \mathrm{E}-01$ & $<1.05 \mathrm{E}-01$ & & MDA & $1.0 \mathrm{E}-03$ \\
\hline $\mathrm{Sb}-126 \mathrm{~m}$ & $<9.91 \mathrm{E}-03$ & $<9.64 \mathrm{E}-02$ & $<1.10 \mathrm{E}-01$ & $<1.08 \mathrm{E}-01$ & $<1.05 \mathrm{E}-01$ & & MDA & $1.0 \mathrm{E}-03$ \\
\hline I-129 & $<5.27 \mathrm{E}-06$ & $1.61 \mathrm{E}-04$ & $1.65 \mathrm{E}-04$ & $1.45 \mathrm{E}-04$ & $1.57 \mathrm{E}-04$ & $1.03 E-05$ & 6.3 & $1.0 \mathrm{E}-04$ \\
\hline Cs-135 & $<2.27 \mathrm{E}-05$ & $2.80 \mathrm{E}-03$ & $2.67 \mathrm{E}-03$ & $2.68 \mathrm{E}-03$ & $2.72 \mathrm{E}-03$ & 7.23E-05 & 21.8 & $5.0 \mathrm{E}-02$ \\
\hline Cs-137 & $<2.74 \mathrm{E}-02$ & $5.50 \mathrm{E}+02$ & $5.90 \mathrm{E}+02$ & $5.32 \mathrm{E}+02$ & $5.57 \mathrm{E}+02$ & $3.00 E+01$ & 5.0 & $1.0 \mathrm{E}-03$ \\
\hline $\mathrm{Ba}-137 \mathrm{~m}$ & $<2.59 \mathrm{E}-02$ & $5.20 \mathrm{E}+02$ & $5.58 \mathrm{E}+02$ & $5.03 \mathrm{E}+02$ & $5.27 \mathrm{E}+02$ & $2.84 E+01$ & 5.0 & $1.0 \mathrm{E}-03$ \\
\hline Pm-147 & $<2.40 \mathrm{E}-02$ & $<1.66 \mathrm{E}+02$ & $<1.59 \mathrm{E}+02$ & $<1.46 \mathrm{E}+02$ & $<1.57 \mathrm{E}+02$ & & UL & None \\
\hline Sm-151 & $<3.07 \mathrm{E}-02$ & $2.11 \mathrm{E}+02$ & $2.03 \mathrm{E}+02$ & $1.86 \mathrm{E}+02$ & $2.00 \mathrm{E}+02$ & $1.31 E+01$ & 16.7 & $3.0 \mathrm{E}+00$ \\
\hline Eu-152 & $<2.73 \mathrm{E}-02$ & $<1.48 \mathrm{E}-01$ & $<1.75 \mathrm{E}-01$ & $<1.71 \mathrm{E}-01$ & $<1.64 \mathrm{E}-01$ & & MDA & $7.0 \mathrm{E}-03$ \\
\hline Eu-154 & $<1.90 \mathrm{E}-02$ & $1.68 \mathrm{E}+01$ & $1.65 \mathrm{E}+01$ & $1.59 \mathrm{E}+01$ & $1.64 \mathrm{E}+01$ & 4.87E-01 & 5.0 & $1.0 \mathrm{E}-03$ \\
\hline Eu-155 & $<2.80 \mathrm{E}-02$ & $3.91 \mathrm{E}+00$ & $5.41 \mathrm{E}+00$ & $4.68 \mathrm{E}+00$ & $4.67 \mathrm{E}+00$ & $7.46 E-01$ & 6.3 & None \\
\hline $\mathrm{Ra}-226^{* *}$ & No yield & $<2.78 \mathrm{E}-03$ & $<3.10 \mathrm{E}-03$ & $<2.55 \mathrm{E}-03$ & $<2.81 \mathrm{E}-03$ & & MDA & $5.0 \mathrm{E}-03$ \\
\hline Ac-227 & No yield & $<3.91 \mathrm{E}-05$ & $<2.23 \mathrm{E}-05$ & $<4.59 \mathrm{E}-05$ & $<3.58 \mathrm{E}-05$ & & UL & $1.30 \mathrm{E}-04$ \\
\hline Th-229 & No yield & 2.99E-05 & 8.33E-05 & $1.18 \mathrm{E}-04$ & $7.71 \mathrm{E}-05$ & $4.44 \mathrm{E}-05$ & 13.0 & $1.0 \mathrm{E}-03$ \\
\hline Th-230 & No yield & $8.24 \mathrm{E}-04$ & 7.07E-04 & $1.00 \mathrm{E}-03$ & 8.45E-04 & $1.50 \mathrm{E}-04$ & 13.0 & $1.0 \mathrm{E}-03$ \\
\hline $\mathrm{Pa}-231$ & $<9.17 \mathrm{E}-04$ & $<7.42 \mathrm{E}-04$ & $<4.72 \mathrm{E}-04$ & $<8.72 \mathrm{E}-04$ & $<6.95 \mathrm{E}-04$ & & DL & $1.0 \mathrm{E}-03$ \\
\hline U-232 & No activity & $7.30 \mathrm{E}-05$ & $9.14 \mathrm{E}-05$ & $5.14 \mathrm{E}-05$ & 7.19E-05 & $2.01 E-05$ & 20.7 & $1.0 \mathrm{E}-03$ \\
\hline U-233 & $<1.21 \mathrm{E}-04$ & $3.20 \mathrm{E}-03$ & $3.28 \mathrm{E}-03$ & $3.24 \mathrm{E}-03$ & $3.24 \mathrm{E}-03$ & $4.00 E-05$ & 20.0 & $1.0 \mathrm{E}-03$ \\
\hline U-234 & $<7.81 \mathrm{E}-05$ & $9.09 \mathrm{E}-03$ & $9.05 \mathrm{E}-03$ & $9.36 \mathrm{E}-03$ & $9.17 \mathrm{E}-03$ & $1.69 E-04$ & 20.0 & $1.0 \mathrm{E}-03$ \\
\hline U-235 & $<2.72 \mathrm{E}-08$ & $4.25 \mathrm{E}-04$ & $4.25 \mathrm{E}-04$ & $4.22 \mathrm{E}-04$ & $4.24 \mathrm{E}-04$ & $1.73 E-06$ & 20.0 & $1.0 \mathrm{E}-04$ \\
\hline U-236 & $<8.13 \mathrm{E}-07$ & $5.14 \mathrm{E}-04$ & $5.40 \mathrm{E}-04$ & $5.25 \mathrm{E}-04$ & $5.26 \mathrm{E}-04$ & $1.31 E-05$ & 20.0 & $1.0 \mathrm{E}-03$ \\
\hline U-238 & $<8.17 \mathrm{E}-07$ & $1.35 \mathrm{E}-02$ & $1.35 \mathrm{E}-02$ & $1.16 \mathrm{E}-02$ & $1.29 \mathrm{E}-02$ & $1.10 E-03$ & 20.0 & $1.0 \mathrm{E}-03$ \\
\hline Np-237 & $<1.48 \mathrm{E}-04$ & $2.41 \mathrm{E}-02$ & $1.59 \mathrm{E}-02$ & $1.42 \mathrm{E}-02$ & $1.81 \mathrm{E}-02$ & $5.29 E-03$ & 24.7 & $1.0 \mathrm{E}-03$ \\
\hline $\mathrm{Pu}-238$ & 2.73E-01 & $3.19 \mathrm{E}+00$ & $3.05 \mathrm{E}+00$ & $3.44 \mathrm{E}+00$ & $3.23 \mathrm{E}+00$ & $1.97 E-01$ & 9.2 & $1.0 \mathrm{E}-03$ \\
\hline $\mathrm{Pu}-239$ & $3.34 \mathrm{E}-02$ & $2.66 \mathrm{E}+00$ & $2.65 \mathrm{E}+00$ & $2.94 \mathrm{E}+00$ & $2.75 E+00$ & $1.65 E-01$ & 20.0 & $1.0 \mathrm{E}-03$ \\
\hline $\mathrm{Pu}-240$ & $<8.26 \mathrm{E}-03$ & $1.63 \mathrm{E}+00$ & $1.67 \mathrm{E}+00$ & $1.86 \mathrm{E}+00$ & $1.72 \mathrm{E}+00$ & $1.23 E-01$ & 20.0 & $1.0 \mathrm{E}-03$ \\
\hline $\mathrm{Pu}-239 / 240$ & $<1.08 \mathrm{E}-01$ & $4.29 \mathrm{E}+00$ & $4.32 \mathrm{E}+00$ & $4.82 \mathrm{E}+00$ & $4.48 \mathrm{E}+00$ & $2.98 E-01$ & 7.8 & None \\
\hline $\mathrm{Pu}-241$ & $<9.50 \mathrm{E}-03$ & $6.26 \mathrm{E}+00$ & $6.31 \mathrm{E}+00$ & $7.12 \mathrm{E}+00$ & $6.56 \mathrm{E}+00$ & $4.82 E-01$ & 15.4 & $1.0 \mathrm{E}-03$ \\
\hline $\mathrm{Pu}-242$ & $<1.43 \mathrm{E}-04$ & $2.01 \mathrm{E}-03$ & $2.01 \mathrm{E}-03$ & $2.25 \mathrm{E}-03$ & $2.09 E-03$ & $1.39 E-04$ & 20.0 & $1.0 \mathrm{E}-03$ \\
\hline Pu-244 & $<6.66 \mathrm{E}-07$ & $<6.24 \mathrm{E}-07$ & $<5.75 \mathrm{E}-07$ & $<6.68 \mathrm{E}-07$ & $<6.22 \mathrm{E}-07$ & & DL & $1.3 \mathrm{E}-04$ \\
\hline Am-241 & $<3.14 \mathrm{E}-02$ & $6.71 \mathrm{E}+01$ & $6.44 \mathrm{E}+01$ & $6.08 \mathrm{E}+01$ & $6.41 \mathrm{E}+01$ & $3.16 E+00$ & 5 & $1.0 \mathrm{E}-03$ \\
\hline Am-242m & $<1.36 \mathrm{E}-02$ & $8.92 \mathrm{E}-02$ & $1.29 \mathrm{E}-01$ & $1.42 \mathrm{E}-01$ & $1.20 \mathrm{E}-01$ & $2.76 E-02$ & 24.1 & $1.0 \mathrm{E}-03$ \\
\hline Np-239 & $<3.34 \mathrm{E}-02$ & 9.19E-01 & $<7.07 \mathrm{E}-01$ & $<7.52 \mathrm{E}-01$ & $\leq 7.93 \mathrm{E}-01$ & & 21.7/MDA & None \\
\hline
\end{tabular}


SRNL-STI-2012-00365, Rev. 0

Page 28 of 94

Table 14 Continued. Radiological Constituents for Tank 6F Composite Sample \#2, $\mu \mathrm{Ci} / \mathrm{g}$.

\begin{tabular}{|c|c|c|c|c|c|c|c|c|}
\hline Am-243 & $<2.19 \mathrm{E}-02$ & $1.52 \mathrm{E}+00$ & $1.35 \mathrm{E}+00$ & $1.36 \mathrm{E}+00$ & $1.41 \mathrm{E}+00$ & $9.52 E-02$ & 13.1 & $1.0 \mathrm{E}-03$ \\
\hline $\mathrm{Cm}-242$ & $<1.13 \mathrm{E}-02$ & 7.39E-02 & $1.07 \mathrm{E}-01$ & $1.18 \mathrm{E}-01$ & 9.97E-02 & $2.30 E-02$ & 24.1 & None \\
\hline $\mathrm{Cm}-243$ & $<3.05 \mathrm{E}-03$ & $<1.10 \mathrm{E}+00$ & $<9.59 \mathrm{E}-01$ & $<8.11 \mathrm{E}-01$ & $<9.58 \mathrm{E}-01$ & & UL & $2.0 \mathrm{E}-02$ \\
\hline $\mathrm{Cm}-244$ & $5.00 \mathrm{E}-01$ & $4.77 \mathrm{E}+01$ & $5.68 \mathrm{E}+01$ & $5.50 \mathrm{E}+01$ & $5.32 \mathrm{E}+01$ & $4.77 E+00$ & 15.07 & None \\
\hline Cm-245 & $<1.27 \mathrm{E}-04$ & $4.59 \mathrm{E}-03$ & $5.68 \mathrm{E}-03$ & $5.27 \mathrm{E}-03$ & 5.18E-03 & $5.46 E-04$ & 20.00 & $2.0 \mathrm{E}-02$ \\
\hline $\mathrm{Cm}-246$ & $<6.35 \mathrm{E}-04$ & $3.23 \mathrm{E}-02$ & $3.76 \mathrm{E}-02$ & $3.85 \mathrm{E}-02$ & $3.61 \mathrm{E}-02$ & $3.37 E-03$ & 20.00 & None \\
\hline Cm-247 & $<5.23 \mathrm{E}-08$ & $<1.66 \mathrm{E}-07$ & $<2.16 \mathrm{E}-07$ & $<2.62 \mathrm{E}-07$ & $<2.15 \mathrm{E}-07$ & & UL & $1.3 \mathrm{E}-04$ \\
\hline Bk-247 & $<5.86 \mathrm{E}-04$ & $<1.85 \mathrm{E}-03$ & $<2.41 \mathrm{E}-03$ & $<2.92 \mathrm{E}-03$ & $<2.39 \mathrm{E}-03$ & & UL & None \\
\hline Cm-248 & $<9.23 \mathrm{E}-06$ & $<9.23 \mathrm{E}-06$ & $<1.14 \mathrm{E}-05$ & $<2.39 \mathrm{E}-05$ & $<1.48 \mathrm{E}-05$ & & UL & $1.3 \mathrm{E}-04$ \\
\hline Cf-249 & $<7.84 \mathrm{E}-04$ & $<4.38 \mathrm{E}-03$ & $<4.82 \mathrm{E}-03$ & $<9.95 \mathrm{E}-03$ & $<6.38 \mathrm{E}-03$ & & MDA & $5.0 \mathrm{E}-03$ \\
\hline Cf-250 & $<7.97 \mathrm{E}-05$ & $<2.68 \mathrm{E}-05$ & $<2.35 \mathrm{E}-05$ & $<1.40 \mathrm{E}-04$ & $<6.33 \mathrm{E}-05$ & & UL & None \\
\hline Cf-251 & $<1.73 \mathrm{E}-03$ & $<9.50 \mathrm{E}-03$ & $<1.05 \mathrm{E}-02$ & $<2.18 \mathrm{E}-02$ & $<1.39 \mathrm{E}-02$ & & MDA & None \\
\hline Cf-252 & $<1.13 \mathrm{E}-02$ & $<7.39 \mathrm{E}-02$ & $<1.07 \mathrm{E}-01$ & $<1.18 \mathrm{E}-01$ & $<9.96 \mathrm{E}-02$ & & UL & None \\
\hline
\end{tabular}

** While analysis of these isotopes is needed, meeting the detection limits for these isotopes is a lower priority than meeting detection limits for the other specified isotopes. 
SRNL-STI-2012-00365, Rev. 0

Page 29 of 94

Table 15 Radiological Constituents for Tank 6F Composite Sample \#3, $\mu \mathrm{Ci} / \mathrm{g}$.

\begin{tabular}{|c|c|c|c|c|c|c|c|c|}
\hline Analytes & Blank & Run 1 & Run 2 & Run 3 & Average & Stdev & \%Uncert. & $\begin{array}{c}\text { Targeted } \\
\text { Minimum } \\
\text { Detection } \\
\text { Limits }\end{array}$ \\
\hline Gross alpha & $<1.92 \mathrm{E}-02$ & $<4.55 \mathrm{E}+02$ & $<2.91 \mathrm{E}+02$ & $<2.36 \mathrm{E}+02$ & $<3.27 \mathrm{E}+02$ & & MDA & None \\
\hline $\begin{array}{c}\text { Non-volatile } \\
\text { beta }\end{array}$ & $6.88 \mathrm{E}-01$ & $4.28 \mathrm{E}+04$ & $4.39 \mathrm{E}+04$ & $4.22 \mathrm{E}+04$ & $4.30 \mathrm{E}+04$ & $8.46 E+02$ & 10.0 & None \\
\hline $\mathrm{H}-3$ & $<2.06 \mathrm{E}-02$ & $<2.17 \mathrm{E}-02$ & $<2.06 \mathrm{E}-02$ & $<2.01 \mathrm{E}-02$ & $<2.08 \mathrm{E}-02$ & & MDA & $1.0 \mathrm{E}-01$ \\
\hline C-14 & $<7.34 \mathrm{E}-04$ & $1.26 \mathrm{E}-02$ & $1.92 \mathrm{E}-02$ & $1.02 \mathrm{E}-02$ & $1.40 \mathrm{E}-02$ & $4.62 \mathrm{E}-03$ & 48.7 & $1.0 \mathrm{E}-01$ \\
\hline Ni-59 & $<3.91 \mathrm{E}-02$ & $5.32 \mathrm{E}+00$ & $5.23 \mathrm{E}+00$ & $5.63 \mathrm{E}+00$ & $5.39 \mathrm{E}+00$ & $2.13 E-01$ & 10.0 & $9.0 \mathrm{E}-02$ \\
\hline Ni-63 & $2.04 \mathrm{E}-01$ & $3.39 \mathrm{E}+02$ & $5.00 \mathrm{E}+02$ & $3.49 \mathrm{E}+02$ & $3.96 \mathrm{E}+02$ & $9.01 E+01$ & 10.0 & $1.0 \mathrm{E}-01$ \\
\hline Co-60 & $<1.02 \mathrm{E}-02$ & $8.42 \mathrm{E}+00$ & $8.78 \mathrm{E}+00$ & $8.15 \mathrm{E}+00$ & $8.45 \mathrm{E}+00$ & $3.16 E-01$ & 5 & $1.0 \mathrm{E}-03$ \\
\hline Se-79 & $<2.80 \mathrm{E}-04$ & $1.25 \mathrm{E}-02$ & $9.41 \mathrm{E}-03$ & $8.15 \mathrm{E}-03$ & $1.00 \mathrm{E}-02$ & $2.22 E-03$ & 24.4 & $1.0 \mathrm{E}-03$ \\
\hline Sr-90 & $<6.58 \mathrm{E}+00$ & $1.68 \mathrm{E}+04$ & $1.50 \mathrm{E}+04$ & $1.76 \mathrm{E}+04$ & $1.65 E+04$ & $1.34 E+03$ & 6.4 & $1.0 \mathrm{E}-03$ \\
\hline Y-90 & $<6.58 \mathrm{E}+00$ & $1.68 \mathrm{E}+04$ & $1.50 \mathrm{E}+04$ & $1.76 \mathrm{E}+04$ & $1.65 E+04$ & $1.34 E+03$ & 6.4 & $1.0 \mathrm{E}-03$ \\
\hline Zr-93 & $6.10 \mathrm{E}-03$ & $1.03 \mathrm{E}+00$ & $8.25 \mathrm{E}-01$ & $8.10 \mathrm{E}-01$ & 8.88E-01 & $1.23 \mathrm{E}-01$ & 27.1 & $1.0 \mathrm{E}-03$ \\
\hline $\mathrm{Nb}-94$ & $<2.90 \mathrm{E}-04$ & $<2.15 \mathrm{E}-03$ & $<1.31 \mathrm{E}-03$ & $<1.59 \mathrm{E}-03$ & $<1.68 \mathrm{E}-03$ & & MDA & $3.0 \mathrm{E}-03$ \\
\hline Tc-99 & $<1.45 \mathrm{E}-04$ & $1.21 \mathrm{E}-01$ & $1.07 \mathrm{E}-01$ & $8.15 \mathrm{E}-02$ & 1.03E-01 & $1.99 E-02$ & 6.3 & $1.0 \mathrm{E}-03$ \\
\hline Sn-126 & $<2.46 \mathrm{E}-02$ & $<6.62 \mathrm{E}-01$ & $<7.97 \mathrm{E}-01$ & $<6.44 \mathrm{E}-01$ & $<7.01 \mathrm{E}-01$ & & MDA & $1.0 \mathrm{E}-03$ \\
\hline Sb-126 & $<9.91 \mathrm{E}-03$ & $<1.17 \mathrm{E}-01$ & $<1.06 \mathrm{E}-01$ & $<1.13 \mathrm{E}-01$ & $<1.12 \mathrm{E}-01$ & & MDA & $1.0 \mathrm{E}-03$ \\
\hline Sb-126m & $<9.91 \mathrm{E}-03$ & $<1.17 \mathrm{E}-01$ & $<1.06 \mathrm{E}-01$ & $<1.13 \mathrm{E}-01$ & $<1.12 \mathrm{E}-01$ & & MDA & $1.0 \mathrm{E}-03$ \\
\hline I-129 & $<5.27 \mathrm{E}-06$ & $1.83 \mathrm{E}-04$ & $1.91 \mathrm{E}-04$ & $1.88 \mathrm{E}-04$ & $1.87 \mathrm{E}-04$ & $4.30 E-06$ & 5.7 & $1.0 \mathrm{E}-04$ \\
\hline Cs-135 & $<2.27 \mathrm{E}-05$ & $2.62 \mathrm{E}-03$ & $2.83 \mathrm{E}-03$ & $2.64 \mathrm{E}-03$ & $2.70 \mathrm{E}-03$ & $1.16 \mathrm{E}-04$ & 20 & $5.0 \mathrm{E}-02$ \\
\hline Cs-137 & $<2.74 \mathrm{E}-02$ & $5.41 \mathrm{E}+02$ & $5.41 \mathrm{E}+02$ & $5.27 \mathrm{E}+02$ & $5.36 \mathrm{E}+02$ & $7.80 E+00$ & 5.0 & $1.0 \mathrm{E}-03$ \\
\hline Ba-137m & $<2.59 \mathrm{E}-02$ & $5.11 \mathrm{E}+02$ & $5.11 \mathrm{E}+02$ & $4.99 \mathrm{E}+02$ & $5.07 E+02$ & $7.38 E+00$ & 5.0 & $1.0 \mathrm{E}-03$ \\
\hline Pm-147 & $<2.40 \mathrm{E}-02$ & $<1.64 \mathrm{E}+02$ & $<1.70 \mathrm{E}+02$ & $<1.64 \mathrm{E}+02$ & $<1.66 \mathrm{E}+02$ & & UL & None \\
\hline Sm-151 & $<3.07 \mathrm{E}-02$ & $2.07 \mathrm{E}+02$ & $2.16 \mathrm{E}+02$ & $2.08 \mathrm{E}+02$ & $2.10 \mathrm{E}+02$ & $5.12 E+00$ & 16.7 & $3.0 \mathrm{E}+00$ \\
\hline Eu-152 & $<2.73 \mathrm{E}-02$ & $<1.91 \mathrm{E}-01$ & $<1.60 \mathrm{E}-01$ & $<1.78 \mathrm{E}-01$ & $<1.76 \mathrm{E}-01$ & & MDA & $7.0 \mathrm{E}-03$ \\
\hline Eu-154 & $<1.90 \mathrm{E}-02$ & $2.05 \mathrm{E}+01$ & $2.14 \mathrm{E}+01$ & $2.00 \mathrm{E}+01$ & $2.06 \mathrm{E}+01$ & $7.28 E-01$ & 5.0 & $1.0 \mathrm{E}-03$ \\
\hline Eu-155 & $<2.80 \mathrm{E}-02$ & $5.59 \mathrm{E}+00$ & $4.59 \mathrm{E}+00$ & $3.63 \mathrm{E}+00$ & $4.60 \mathrm{E}+00$ & $9.80 E-01$ & 7.7 & None \\
\hline $\mathrm{Ra}-226 * *$ & No yield & $<3.57 \mathrm{E}-03$ & $<3.19 \mathrm{E}-03$ & $<4.35 \mathrm{E}-03$ & $<3.70 \mathrm{E}-03$ & & MDA & $5.0 \mathrm{E}-03$ \\
\hline Ac-227 & No yield & Poor yield & $<3.41 \mathrm{E}-05$ & $<4.39 \mathrm{E}-05$ & $<3.90 \mathrm{E}-05$ & & UL & $1.30 \mathrm{E}-04$ \\
\hline Th-229 & No yield & Poor yield & $9.05 \mathrm{E}-05$ & $1.25 \mathrm{E}-04$ & 1.08E-04 & $2.45 E-05$ & 12.9 & $1.0 \mathrm{E}-03$ \\
\hline Th-230 & No yield & $1.18 \mathrm{E}-03$ & $8.38 \mathrm{E}-04$ & $1.18 \mathrm{E}-03$ & $1.07 E-03$ & $1.98 E-04$ & 10.61 & $1.0 \mathrm{E}-03$ \\
\hline $\mathrm{Pa}-231$ & $<9.17 \mathrm{E}-04$ & $<1.02 \mathrm{E}-03$ & $<5.23 \mathrm{E}-04$ & $<9.69 \mathrm{E}-04$ & $<8.37 \mathrm{E}-04$ & & $\mathrm{DL}$ & $1.0 \mathrm{E}-03$ \\
\hline U-232 & No activity & $8.87 \mathrm{E}-05$ & $4.55 \mathrm{E}-05$ & $8.92 \mathrm{E}-05$ & $7.45 \mathrm{E}-05$ & $2.51 E-05$ & 21.60 & $1.0 \mathrm{E}-03$ \\
\hline U-233 & $<1.21 \mathrm{E}-04$ & $3.56 \mathrm{E}-03$ & $3.20 \mathrm{E}-03$ & $3.77 \mathrm{E}-03$ & $3.51 \mathrm{E}-03$ & $2.88 E-04$ & 20.0 & $1.0 \mathrm{E}-03$ \\
\hline U-234 & $<7.81 \mathrm{E}-05$ & $1.01 \mathrm{E}-02$ & $9.38 \mathrm{E}-03$ & $1.11 \mathrm{E}-02$ & 1.02E-02 & $8.64 E-04$ & 20.0 & $1.0 \mathrm{E}-03$ \\
\hline U-235 & $<2.72 \mathrm{E}-08$ & $4.70 \mathrm{E}-04$ & $4.29 \mathrm{E}-04$ & $5.09 \mathrm{E}-04$ & $4.69 \mathrm{E}-04$ & $4.00 E-05$ & 20.0 & $1.0 \mathrm{E}-04$ \\
\hline U-236 & $<8.13 \mathrm{E}-07$ & $5.61 \mathrm{E}-04$ & $5.16 \mathrm{E}-04$ & $6.20 \mathrm{E}-04$ & $5.66 \mathrm{E}-04$ & $5.22 E-05$ & 20.0 & $1.0 \mathrm{E}-03$ \\
\hline U-238 & $<8.17 \mathrm{E}-07$ & $1.57 \mathrm{E}-02$ & $1.60 \mathrm{E}-02$ & $1.63 \mathrm{E}-02$ & $1.60 \mathrm{E}-02$ & $3.00 E-04$ & 20.0 & $1.0 \mathrm{E}-03$ \\
\hline Np-237 & $<1.48 \mathrm{E}-04$ & $2.56 \mathrm{E}-02$ & $2.38 \mathrm{E}-02$ & $3.37 \mathrm{E}-02$ & $2.77 \mathrm{E}-02$ & $5.27 E-03$ & 24.0 & $1.0 \mathrm{E}-03$ \\
\hline Pu-238 & 2.73E-01 & $3.52 \mathrm{E}+00$ & $3.57 \mathrm{E}+00$ & $3.16 \mathrm{E}+00$ & $3.42 \mathrm{E}+00$ & $2.21 E-01$ & 9.5 & $1.0 \mathrm{E}-03$ \\
\hline $\mathrm{Pu}-239$ & 3.34E-02 & $3.29 \mathrm{E}+00$ & $2.99 \mathrm{E}+00$ & $2.88 \mathrm{E}+00$ & $3.05 E+00$ & $2.12 E-01$ & 20.0 & $1.0 \mathrm{E}-03$ \\
\hline $\mathrm{Pu}-240$ & $<8.26 \mathrm{E}-03$ & $2.05 \mathrm{E}+00$ & $1.86 \mathrm{E}+00$ & $1.78 \mathrm{E}+00$ & $1.90 \mathrm{E}+00$ & $1.39 E-01$ & 20.0 & $1.0 \mathrm{E}-03$ \\
\hline $\mathrm{Pu}-239 / 240$ & $<1.08 \mathrm{E}-01$ & $5.32 \mathrm{E}+00$ & $4.86 \mathrm{E}+00$ & $4.64 \mathrm{E}+00$ & $4.94 \mathrm{E}+00$ & $3.44 E-01$ & 7.6 & None \\
\hline Pu-241 & $<9.50 \mathrm{E}-03$ & $7.48 \mathrm{E}+00$ & $7.03 \mathrm{E}+00$ & $6.62 \mathrm{E}+00$ & $7.04 \mathrm{E}+00$ & $4.28 E-01$ & 15.4 & $1.0 \mathrm{E}-03$ \\
\hline $\mathrm{Pu}-242$ & $<1.43 \mathrm{E}-04$ & $2.41 \mathrm{E}-03$ & $2.23 \mathrm{E}-03$ & $2.09 \mathrm{E}-03$ & $2.24 \mathrm{E}-03$ & $1.60 E-04$ & 20.0 & $1.0 \mathrm{E}-03$ \\
\hline Pu-244 & $<6.66 \mathrm{E}-07$ & $<6.44 \mathrm{E}-07$ & $<7.76 \mathrm{E}-07$ & $<5.75 \mathrm{E}-07$ & $<6.65 \mathrm{E}-07$ & & DL & $1.3 \mathrm{E}-04$ \\
\hline Am-241 & $<3.14 \mathrm{E}-02$ & $8.02 \mathrm{E}+1$ & $8.56 \mathrm{E}+01$ & $7.84 \mathrm{E}+01$ & $8.14 \mathrm{E}+01$ & $3.75 E+00$ & 5 & $1.0 \mathrm{E}-03$ \\
\hline Am-242m & $<1.36 \mathrm{E}-02$ & $1.26 \mathrm{E}-01$ & $1.14 \mathrm{E}-01$ & $1.33 \mathrm{E}-01$ & $1.24 \mathrm{E}-01$ & $9.76 E-03$ & 30.3 & $1.0 \mathrm{E}-03$ \\
\hline Np-239 & $<3.34 \mathrm{E}-02$ & $1.56 \mathrm{E}+00$ & $1.13 \mathrm{E}+00$ & $1.62 \mathrm{E}+00$ & $1.44 \mathrm{E}+00$ & $2.67 E-01$ & 11.4 & None \\
\hline
\end{tabular}


Table 15 Continued. Radiological Constituents for Tank 6F Composite Sample \#3, $\mu \mathrm{Ci} / \mathrm{g}$.

\begin{tabular}{|c|c|c|c|c|c|c|c|c|}
\hline Am-243 & $<2.19 \mathrm{E}-02$ & $1.88 \mathrm{E}+00$ & $1.77 \mathrm{E}+00$ & $1.88 \mathrm{E}+00$ & $1.85 \mathrm{E}+00$ & $6.12 \mathrm{E}-02$ & 11.2 & $1.0 \mathrm{E}-03$ \\
\hline $\mathrm{Cm}-242$ & $<1.13 \mathrm{E}-02$ & $1.04 \mathrm{E}-01$ & $9.41 \mathrm{E}-02$ & $1.10 \mathrm{E}-01$ & 1.03E-01 & $8.17 E-03$ & 30.32 & None \\
\hline Cm-243 & $<3.05 \mathrm{E}-03$ & $<1.25 \mathrm{E}+00$ & $<1.45 \mathrm{E}+00$ & $<1.32 \mathrm{E}+00$ & $<1.34 \mathrm{E}+00$ & & UL & $2.0 \mathrm{E}-02$ \\
\hline $\mathrm{Cm}-244$ & $5.00 \mathrm{E}-01$ & $6.62 \mathrm{E}+01$ & $6.26 \mathrm{E}+01$ & $6.35 \mathrm{E}+01$ & $6.41 \mathrm{E}+01$ & $1.88 E+00$ & 15.03 & None \\
\hline $\mathrm{Cm}-245$ & $<1.27 \mathrm{E}-04$ & $6.40 \mathrm{E}-03$ & $6.17 \mathrm{E}-03$ & $6.71 \mathrm{E}-03$ & $6.43 \mathrm{E}-03$ & $2.72 E-04$ & 20.00 & $2.0 \mathrm{E}-02$ \\
\hline Cm-246 & $<6.35 \mathrm{E}-04$ & $4.37 \mathrm{E}-02$ & $4.17 \mathrm{E}-02$ & $4.23 \mathrm{E}-02$ & $4.25 \mathrm{E}-02$ & $1.04 E-03$ & 20.00 & None \\
\hline $\mathrm{Cm}-247$ & $<5.23 \mathrm{E}-08$ & $<2.39 \mathrm{E}-07$ & $<2.76 \mathrm{E}-07$ & $<2.16 \mathrm{E}-07$ & $<2.44 \mathrm{E}-07$ & & UL & $1.3 \mathrm{E}-04$ \\
\hline Bk-247 & $<5.86 \mathrm{E}-04$ & $<2.67 \mathrm{E}-03$ & $<3.08 \mathrm{E}-03$ & $<2.41 \mathrm{E}-03$ & $<2.72 \mathrm{E}-03$ & & UL & None \\
\hline Cm-248 & $<9.23 \mathrm{E}-06$ & $<8.33 \mathrm{E}-06$ & $<9.10 \mathrm{E}-06$ & $<1.42 \mathrm{E}-05$ & $<1.06 \mathrm{E}-05$ & & UL & $1.3 \mathrm{E}-04$ \\
\hline Cf-249 & $<7.84 \mathrm{E}-04$ & $<6.53 \mathrm{E}-03$ & $<5.45 \mathrm{E}-03$ & $<8.56 \mathrm{E}-03$ & $<6.85 \mathrm{E}-03$ & & MDA & $5.0 \mathrm{E}-03$ \\
\hline Cf-250 & $<7.97 \mathrm{E}-05$ & $<3.49 \mathrm{E}-05$ & $<2.34 \mathrm{E}-05$ & $<6.17 \mathrm{E}-05$ & $<4.00 \mathrm{E}-05$ & & $\mathrm{UL}$ & None \\
\hline Cf-251 & $<1.73 \mathrm{E}-03$ & $<1.69 \mathrm{E}-02$ & $<1.19 \mathrm{E}-02$ & $<2.26 \mathrm{E}-02$ & $<1.71 \mathrm{E}-02$ & & MDA & None \\
\hline Cf-252 & $<1.13 \mathrm{E}-02$ & $<1.04 \mathrm{E}-01$ & $<9.41 \mathrm{E}-02$ & $<1.10 \mathrm{E}-01$ & $<1.03 \mathrm{E}-01$ & & $\mathrm{UL}$ & None \\
\hline
\end{tabular}

** While analysis of these isotopes is needed, meeting the detection limits for these isotopes is a lower priority than meeting detection limits for the other specified isotopes.

\subsection{Statistical Analyses}

All statistical data manipulation, conclusions and recommendations on select radionuclide and inorganic components of Tank 6F analyses results are presented in Appendix C.

\subsection{CONCLUSIONS}

Tank 6F composite samples were analyzed for radiological, elemental and chemical constituents. Where analytical methods yielded additional contaminants other than those requested by the customer, these results are also reported.

The target detection limits for isotopes analyzed were based on customer desired detection limits as specified in the technical task request documents. While many of the target detection limits, as specified in the Technical Task Request and Task Technical and Quality Assurance Plans were met for the species characterized for Tank $6 \mathrm{~F}$ composite samples, some were not met. In a number of cases, the relatively high levels of radioactive species of the same element or a chemically similar element precluded the ability to measure some isotopes to low levels. The isotopes whose minimum detection limits were not met in all cases included the following: Sn126, Sb-126, Sb-126m, Eu-152, Cm-243 and Cf-249. However, SRNL, in conjunction with the customer, reviewed the few cases where the detection limit goals were not met and determined that the impacts were acceptable 5 .

All of the inorganic constituents displayed heterogeneity as evidenced by the ANOVA results. The inorganic results demonstrated consistent differences across the composite samples: lowest concentrations for Composite Sample 1, intermediate-valued concentrations for Composite Sample 2, and highest concentrations for Composite Sample 3. The Hg and Mo results suggest possible measurement outliers. However, the magnitudes of the differences between the $\mathrm{Hg}$ UCL95 results and the magnitudes of the differences between the Mo UCL95 results not appear to have practical significance. It is recommended to remove the potential measurement outliers. Doing so is conservative in the sense of producing a higher UCL95 for Hg and Mo than if the potential outliers were included in the calculations. 
In contrast to the inorganic results, most of the radionuclides did not demonstrate heterogeneity among the composite sample results.

\subsection{QUALITY ASSURANCE}

The Task Technical and Quality Assurance Plan details the planned activities and associated quality assurance implementing procedures for the characterization of Tank 6F (TTQAP ${ }^{9}$ ). Laboratory Notebooks SRNL-NB-2011-00125, SRNL-NB-2011-00089, WSRC-NB-2001-00142 and various $\mathrm{AD}$ notebooks contain the experimental and analytical data.

\subsection{ACKNOWLEDGEMENTS}

The authors thank Analytical Development Section personnel, in particular, Mark Jones, and Cecilia Diprete, for performing actinide and other metal analyses. We also thank the shielded cell operations personnel, in particular, Linda Bush, Carl Black and Kevin Reid for their work with the Tank $6 \mathrm{~F}$ samples in the shielded cells.

\subsection{REFERENCES}

1. B. Dean, "Tank 5 Composite Sample Volumetric Proportions," SRR-CWDA-2011-00067, Rev.1 April 20, 2011

2. B. Martin, "Justification for Adjustments to the Tank 6 Sample Plan" SRR-CWDA-201100159, Revision 0, October 2011.

3. C. J Coleman, R. A. Dewberry, M. F. Bryant and J.J Gemmill," SRL's Performance in Round Robin \#6-Analyses of Simulated Defense Waste Glass', WSRC-TR-91-187, Rev. 0, May 31, 1991.

4. D. Koopman,'Tank 8, Drum 1, Sludge simulant, SRTC mobile Lab. ID \# 20000616," July 26,2000

5. A. W. Wiggins, Jr., "Detection Limit Acceptability for Tank 6 Residual Sample Analysis" SRR-CWDA-2012-00091, June 7, 2012.

6. Technical Task Request number HLE-TTR-2010-004, Rev 7

7. Task Technical Quality Assurance Plan for the Analysis of the Tank 5F and Tank 6F Final Characterization Samples-SRNL-RP-2010-01695, Rev. 1

8. Tank 6F Sampling and Analysis Plan-SRR-LWE-2010-00285, Nov. 2011, Rev. 6

9. Task Technical Quality Assurance Plan and Characterization Plan for Tanks 18F and 19F Solid Fraction Analysis - 2008, SRNL-RP-2008-01202, Rev. 1, July 10, 2009 
SRNL-STI-2012-00365, Rev. 0

Page 32 of 94

\section{APPENDIX A-1 AD TRACKING NUMBERS FOR TANK 6F CHARACTERIZATION}

\begin{tabular}{|c|c|c|}
\hline Analytes & Method (s) & $\begin{array}{c}\text { SRNL AD Tracking } \\
\text { Number (LIMS) }\end{array}$ \\
\hline $\begin{array}{l}\text { Total } \\
\text { Alpha }\end{array}$ & Rad Screen & $\begin{array}{l}300295756-300295758 \\
300295762-300295764\end{array}$ \\
\hline Non-volatile Beta & Rad Screen & $\begin{array}{l}300295765-300295767 \\
300295768-300295770\end{array}$ \\
\hline Sr-90 & Sr90 & $\begin{array}{l}300295756-300295764 \\
300295766-300295769\end{array}$ \\
\hline $\mathrm{Pu}-238$ & $\mathrm{Pu}-238 / 241$ & 300295756-300295769 \\
\hline $\mathrm{Pu}-241$ & Pu-238/241 & $300295756-300295769$ \\
\hline Cs-137 & GAMMA SPEC & $300295756-300295766$ \\
\hline $\mathrm{U}-232$ & U-232 & $300295756-300295767$ \\
\hline U-233 & U-233, U-234, U-235, U-236 & $300295756-300295767$ \\
\hline $\mathrm{U}-234$ & U-233, U-234, U-235, U-236 & $300295756-300295767$ \\
\hline U-235 & U-233, U-234, U-235, U-236 & $300295756-300295767$ \\
\hline $\mathrm{U}-236$ & U-233, U-234, U-235, U-236 & $300295756-300295767$ \\
\hline U-238 & ICp-MS & $300295756-300295767$ \\
\hline Co-60 & GAMMA SPEC Cs REMOVED & $300295762-300295766$ \\
\hline Sb-126 & GAMMA SPEC Cs REMOVED & $300295762-300295766$ \\
\hline Eu-154 & GAMMA SPEC Cs REMOVED & $300295762-300295766$ \\
\hline Eu-155 & GAMMA SPEC Cs REMOVED & $300295762-300295766$ \\
\hline Am-241 & Gamma Spec. & $300295762-300295766$ \\
\hline $\mathrm{Cm}-242$ & $\mathrm{Am} / \mathrm{Cm}$ & $300295762-300295766$ \\
\hline $\mathrm{Cm}-244$ & $\mathrm{Am} / \mathrm{Cm}$ & $300295762-300295766$ \\
\hline $\mathrm{Pu}-239$ & & $300295756-300295768$ \\
\hline $\mathrm{Pu}-240$ & & $300295756-300295768$ \\
\hline $\mathrm{Pu}-242$ & Pu-242/244 & $300295756-300295768$ \\
\hline $\mathrm{Pu}-244$ & $\mathrm{Pu}-242 / 244$ & $300295756-300295768$ \\
\hline $\mathrm{Pu}-239 / 240$ & Pu-TTA & $300295756-300295769$ \\
\hline PM-147/ SM-151 & $\mathrm{Pm}-147 / \mathrm{Sm}-151$ & $300295756-300295767$ \\
\hline Tritium & TRITIUM & 300295771-300295780 \\
\hline $\mathrm{Ni}-59$ & $\mathrm{Ni}-59,63$ & 300295771-300295783 \\
\hline $\mathrm{Ni}-63$ & $\mathrm{Ni}-59,63$ & $300295771-300295783$ \\
\hline Tc-99 & Tc-99 & 300296139-300296151 \\
\hline $\mathrm{I}-129$ & $\mathrm{I}-129$ & $300296467-300296477$ \\
\hline Cs-135 & Cs-135 & 300295756-300295768 \\
\hline Carbon-14 & Carbon-14 & $300299257-300299386$ \\
\hline Carbon-14, blank & Carbon-14 & $300299268 \& 300299387$ \\
\hline Se-79 & Se-79 & $300297058-300297067$ \\
\hline Zr-93 & Zr-93 & $300295756-300295764$ \\
\hline Zr-93 blank & Zr-93 & $300295765 \& 300295768$ \\
\hline $\mathrm{Am} / \mathrm{Cm}$ & $\mathrm{Am} / \mathrm{Cm}$ & $300296591-300296599$ \\
\hline $\mathrm{Am} / \mathrm{Cm}$ & $\mathrm{Am} / \mathrm{Cm}$ & $300296600-300296601$ \\
\hline
\end{tabular}


SRNL-STI-2012-00365, Rev. 0

Page 33 of 94

\section{APPENDIX A-2 AD TRACKING NUMBERS FOR TANK 6F CHARACTERIZATIONS - CONTINUED}

\begin{tabular}{|c|c|c|c|}
\hline Analytes & Method (s) & $\begin{array}{l}\text { SRNL AD Tracking } \\
\text { Number (LIMS) }\end{array}$ & Comments \\
\hline Nb-94 & Nb-94 & $300295756-300295768$ & \\
\hline Cs-removed gamma & Cs-removed gamma & $300295756-300295766$ & \\
\hline Am/Cm & Am/Cm & $300296591-3002966601$ & \\
\hline Ra-226 & Ra-226 & $300297708-300297726$ & \\
\hline Th-229/230 & Th-229/230 & $30029750-300297869$ & \\
\hline $\mathrm{Ac}-227$ & Ac-227 & $300297850-300297867$ & \\
\hline $\mathrm{Pa}-231$ & Pa-231 & $300297206-300297224$ & \\
\hline $\mathrm{I}-127$ stable) & Mass scpect. & $300297358-300297367$ & Hg in Samples \\
\hline $\mathrm{Np}-237$ & & $300295756-300295770$ & Hg Blank \\
\hline $\mathrm{Hg}$ & CVAA Hg & $300295771-300295779$ & Se Blank \\
\hline & & $300295782-300295783$ & As in Samples \\
\hline $\mathrm{Se}$ & AASe & $300295771-300295779$ & As Blank \\
\hline As & & $300295780-300295785$ & AQR digestions \\
\hline Aations & & $300295771-300295779$ & AQR digestions-ARG \\
\hline & ICP-ES & $300295780-300295785$ & AQR digestions-simulant \\
\hline & & $300295771-300295779$ & PF digestions \\
\hline Cations & ICP-ES & $300295783-300295785$ & PF digestions-ARG \\
\hline & & $300295780-300295786-300295767-$ & PF digestions-simulant \\
\hline
\end{tabular}

\section{APPENDIX A-3. CHEMICAL COMPOSITION FOR REFERENCE TANK 8 SIMULANT SLUDGE}

\begin{tabular}{|c|c|c|c|c|}
\hline & $\begin{array}{l}\text { Analytical Results for } \\
\text { Tank } 8 \text { Simulant Sludge }\end{array}$ & $\begin{array}{l}\text { Standard } \\
\text { deviation }\end{array}$ & $\begin{array}{c}\text { Nominal Recipe for Tank } 8 \\
\text { Simulant Sludge }\end{array}$ & $\begin{array}{c}\text { Percent Relative } \\
\text { Deviation }\end{array}$ \\
\hline & Average & & & \%RD \\
\hline Constituent & wt. $\%$ & & wt. \% & \\
\hline $\mathrm{Al}$ & $8.9 \mathrm{E}+00$ & $1.76 E-01$ & $9.0 \mathrm{E}+00$ & 1.1 \\
\hline $\mathrm{Ba}$ & 2.2E-01 & $4.00 E-03$ & $2.4 \mathrm{E}-01$ & 8.7 \\
\hline $\mathrm{Ca}$ & $2.0 \mathrm{E}+00$ & $2.52 E-02$ & $2.1 \mathrm{E}+00$ & 4.9 \\
\hline${ }^{*} \mathrm{Cr}$ & 2.3E-01 & $4.04 E-03$ & $2.4 \mathrm{E}-01$ & 1.6 \\
\hline $\mathrm{Cu}$ & $1.2 \mathrm{E}-01$ & $4.58 E-03$ & $1.3 \mathrm{E}-01$ & 8.0 \\
\hline $\mathrm{Fe}$ & $2.3 \mathrm{E}+01$ & $3.61 E-01$ & $2.4 \mathrm{E}+01$ & 4.3 \\
\hline $\mathrm{Mg}$ & 1.2E-01 & $2.00 E-03$ & 1.2E-01 & 0.0 \\
\hline $\mathrm{Mn}$ & $2.5 \mathrm{E}+00$ & $5.13 E-02$ & $2.7 \mathrm{E}+00$ & 7.7 \\
\hline $\mathrm{Na}$ & $5.2 \mathrm{E}+00$ & $8.02 E-02$ & $7.4 \mathrm{E}+00$ & 34.9 \\
\hline $\mathrm{Ni}$ & $2.6 \mathrm{E}+00$ & $4.04 \mathrm{E}-02$ & $2.8 \mathrm{E}+00$ & 7.4 \\
\hline$* \mathrm{Si}$ & $7.5 \mathrm{E}-01$ & 5.03E-03 & $7.6 \mathrm{E}-01$ & 1.3 \\
\hline $\mathrm{Sr}$ & $8.3 E-02$ & $1.43 E-03$ & $9.0 \mathrm{E}-02$ & 8.1 \\
\hline $\mathrm{Zn}$ & $2.4 \mathrm{E}-01$ & $7.64 E-03$ & $2.7 \mathrm{E}-01$ & 11.8 \\
\hline
\end{tabular}

* Peroxide fusion digestion data; all other data from aqua regia digestions. 
SRNL-STI-2012-00365, Rev. 0

Page 34 of 94

\section{APPENDIX A-4. CHEMICAL COMPOSITION OF ANALYZED REFERENCE GLASS}

\begin{tabular}{|c|c|c|c|c|}
\hline & $\begin{array}{c}\text { Analytical Results for } \\
\text { Reference Glass } \\
\text { (ARG) }\end{array}$ & Standard deviation & $\begin{array}{c}\text { Nominal Recipe for } \\
\text { Reference Glass } \\
\text { (ARG) }\end{array}$ & $\begin{array}{c}\text { Percent Relative } \\
\text { Deviation }\end{array}$ \\
\hline & Average & & & $\%$ RD \\
\hline Constituent & wt. $\%$ & & wt. $\%$ & \\
\hline $\mathrm{Al}$ & $2.5 \mathrm{E}+00$ & $2.00 E-02$ & $2.5 \mathrm{E}+00$ & 0.0 \\
\hline $\mathrm{B}$ & $2.6 \mathrm{E}+00$ & $2.89 E-02$ & $2.7 \mathrm{E}+00$ & 0.9 \\
\hline $\mathrm{Ba}$ & 8.0E-02 & $7.21 E-04$ & $7.9 \mathrm{E}-02$ & 0.3 \\
\hline $\mathrm{Ca}$ & $1.10 \mathrm{E}+00$ & $1.0 E-02$ & $1.02 \mathrm{E}+00$ & 1.9 \\
\hline $\mathrm{Cr}$ & $6.5 \mathrm{E}-02$ & $1.95 E-03$ & $6.4 \mathrm{E}-02$ & 0.4 \\
\hline${ }^{*} \mathrm{Cu}$ & $<1.2 \mathrm{E}-02$ & & 3.0E-03 & \\
\hline $\mathrm{Fe}$ & $9.9 \mathrm{E}+00$ & $4.00 E-02$ & $9.8 \mathrm{E}+00$ & 1.0 \\
\hline $\mathrm{K}$ & $2.1 \mathrm{E}+00$ & $8.0 E-02$ & $2.3 \mathrm{E}+00$ & 9.1 \\
\hline $\mathrm{Li}$ & $1.5 \mathrm{E}+00$ & $1.00 E-02$ & $1.5 \mathrm{E}+00$ & 0.0 \\
\hline $\mathrm{Mg}$ & $5.1 \mathrm{E}-01$ & $5.57 E-03$ & $5.2 \mathrm{E}-01$ & 1.9 \\
\hline $\mathrm{Mn}$ & $1.5 \mathrm{E}+00$ & $2.00 E-02$ & $1.5 \mathrm{E}+00$ & 0.0 \\
\hline $\mathrm{Na}$ & $8.4 \mathrm{E}+00$ & $1.45 E-01$ & $8.5 \mathrm{E}+00$ & 1.2 \\
\hline $\mathrm{Ni}$ & $8.4 E-01$ & $1.23 E-02$ & 8.3E-01 & 1.2 \\
\hline $\mathrm{P}$ & $1.1 \mathrm{E}-01$ & $0 E+00$ & 1.1E-01 & 0.0 \\
\hline $\mathrm{Si}$ & $2.3 \mathrm{E}+01$ & $1.73 E-01$ & $2.2 \mathrm{E}+01$ & 1.1 \\
\hline$* \mathrm{Sr}$ & 3.3E-03 & $7.37 E-05$ & 3.0E-03 & 9.5 \\
\hline$* \mathrm{Ti}$ & 5.7.-01 & $9.87 E-03$ & $6.9 \mathrm{E}-01$ & 19 \\
\hline $\mathrm{Zn}$ & $1.7 \mathrm{E}-02$ & $2.31 E-04$ & 1.6E-02 & 6.1 \\
\hline
\end{tabular}

* Peroxide fusion digestion data; all other data from aqua regia digestions. 


\section{APPENDIX A-5. ICPES AND ICP-MS COMPARISONS FOR SELECT ANALYTES.}

\section{Natural Lanthanum concentration by MS}

Main stable La isotope used for calculations is mass 139

[Sum of MS signals from mass 139]

Tank 6F Composite No. 1, unit of ug/g sample

Mass $139 \quad 911 \mathrm{ug} / \mathrm{g}=0.911 \mathrm{mg} / \mathrm{g}$

\begin{tabular}{|c|c|c|c|c|}
\hline & $\begin{array}{l}300295756 \\
\text { COMPOSITE 1- } \\
1-\mathrm{PF}, \mathrm{mg} / \mathrm{g}\end{array}$ & $\begin{array}{l}300295757 \\
\text { COMPOSITE } \\
1-2-P F, \mathrm{mg} / \mathrm{g}\end{array}$ & $\begin{array}{l}300295758 \\
\text { COMPOSITE } \\
1-3-P F, \mathrm{mg} / \mathrm{g}\end{array}$ & Averages \\
\hline La by ICP-MS & 0.911 & 0.912 & 0.904 & 0.909 \\
\hline La by ICP-ES & 0.841 & 0.856 & 0.830 & 0.842 \\
\hline \%RD & 8.0 & 6.3 & 8.5 & 7.6 \\
\hline
\end{tabular}

The average percent relative deviation for lanthanum concentrations based on ICP-MS and ICPES is $7.6 \%$.

\section{Natural barium concentration by MS}

Main stable $\mathrm{Ba}$ isotopes used for calculations are masses, 138, 137, 135

[Sum of MS signals from masses 138, 137,135

Tank 6F Composite No. 1, unit of ug/g sample

Mass $138 \quad 942 \mathrm{ug} / \mathrm{g}=0.942 \mathrm{mg} / \mathrm{g}$

Mass $137 \quad 132 \mathrm{ug} / \mathrm{g}=0.132 \mathrm{mg} / \mathrm{g}$

Mass $135 \quad 4.34 \mathrm{ug} / \mathrm{g}=0.0043 \mathrm{mg} / \mathrm{g}$

\begin{tabular}{|l|c|c|c|c|}
\hline & $\begin{array}{l}300295756 \\
\text { COMPOSITE 1- } \\
1-\mathrm{PF}, \mathrm{mg} / \mathrm{g}\end{array}$ & $\begin{array}{l}300295757 \\
\text { COMPOSITE } \\
\text { 1-2-PF, } \mathrm{mg} / \mathrm{g}\end{array}$ & $\begin{array}{l}300295758 \\
\text { COMPOSITE 1- } \\
\text { 3-PF, } \mathrm{mg} / \mathrm{g}\end{array}$ & Averages \\
\hline Ba by ICP-MS & 1.08 & 1.08 & 1.07 & 1.08 \\
\hline Ba by ICP-ES & 1.11 & 1.11 & 1.10 & 1.11 \\
\hline$\%$ RD & 2.7 & 2.7 & 2.8 & 2.7 \\
\hline
\end{tabular}

The average percent relative deviation for barium concentrations based on ICP-MS and ICP-ES is $2.7 \%$.

\section{Natural lead concentration by MS}

Main stable $\mathrm{Pb}$ isotopes used for calculations are masses, 204, 206, 207, 208

[Sum of MS signals from masses 204, 206, 207, 208

Tank 6F Composite No. 1, unit of ug/g sample

Mass $204 \quad 43.1 \mathrm{ug} / \mathrm{g}=0.043 \mathrm{mg} / \mathrm{g}$

Mass $206 \quad 118 \mathrm{ug} / \mathrm{g}=0.118 \mathrm{mg} / \mathrm{g}$

Mass $207 \quad 108 \mathrm{ug} / \mathrm{g}=0.108 \mathrm{mg} / \mathrm{g}$

Mass $208 \quad 243 \mathrm{ug} / \mathrm{g}=0.243$ 
SRNL-STI-2012-00365, Rev. 0

Page 36 of 94

\begin{tabular}{|l|c|c|c|c|}
\hline & $\begin{array}{l}\text { 300295756 } \\
\text { COMPOSITE 1- } \\
\text { 1-PF, mg/g }\end{array}$ & $\begin{array}{l}\text { 300295757 } \\
\text { COMPOSITE } \\
\text { 1-2-PF, } \mathrm{mg} / \mathrm{g}\end{array}$ & $\begin{array}{l}\text { 300295758 } \\
\text { COMPOSITE 1- } \\
\text { 3-PF, } \mathrm{mg} / \mathrm{g}\end{array}$ & Averages \\
\hline $\mathrm{Pb}$ by ICP-MS & 0.512 & 0.540 & 0.523 & 0.525 \\
\hline $\mathrm{Pb}$ by ICP-ES & 0.645 & 0.507 & 0.674 & 0.609 \\
\hline \%RD & 23.0 & 6.3 & 25.2 & $\mathbf{1 4 . 8}$ \\
\hline
\end{tabular}

The average percent relative deviation for lead concentrations based on ICP-MS and ICP-ES is $14.8 \%$.

Table A5-1 AVERAGED CESIUM REMOVED GAMMA AND AM/CM FOR AM-241 AND AM-243.

\begin{tabular}{|c|c|c|c|c|c|c|c|c|c|}
\hline \multirow{2}{*}{$\begin{array}{c}\text { AD LIMS \# } \\
300-\end{array}$} & \multicolumn{7}{|c|}{ Radiological Constituents for Tank 6F Composite Sample \#1, $\mu \mathrm{Ci} / \mathrm{g}$. } & \multirow{2}{*}{$\begin{array}{c}\text { pooled } \\
\text { stat. }\end{array}$} & \multirow[t]{2}{*}{ Comments } \\
\hline & & Blank & Run-1 & Run-2 & Run-3 & Average & Stdev. & & \\
\hline $295756-295758$ & Am-241 & $<3.14 \mathrm{E}-02$ & $5.68 \mathrm{E}+01$ & $6.53 \mathrm{E}+01$ & $5.77 \mathrm{E}+01$ & $5.99 E+01$ & $4.7 E+00$ & 5 & Cs-removed \\
\hline \multirow[t]{2}{*}{$296591-296593$} & Am-241 & $6.62 \mathrm{E}-02$ & $6.35 \mathrm{E}+01$ & $6.39 \mathrm{E}+01$ & $6.17 \mathrm{E}+01$ & $6.30 \mathrm{E}+01$ & $1.19 E+00$ & 5 & $\mathrm{Am} / \mathrm{Cm}$ \\
\hline & Am-241 & $\leq 4.88 \mathrm{E}-02$ & $6.02 \mathrm{E}+01$ & $6.46 \mathrm{E}+01$ & $5.97 \mathrm{E}+01$ & $6.15 \mathrm{E}+01$ & $2.74 E+00$ & 5 & Average \\
\hline $295756-295758$ & Am-243 & $<2.19 \mathrm{E}-02$ & $1.22 \mathrm{E}+00$ & $1.03 \mathrm{E}+00$ & $1.23 \mathrm{E}+00$ & $1.16 \mathrm{E}+00$ & $1.16 E-01$ & 13.3 & Cs-removed \\
\hline \multirow[t]{3}{*}{$296591-296593$} & Am-243 & $<2.82 \mathrm{E}-03$ & $1.14 \mathrm{E}+00$ & $1.14 \mathrm{E}+00$ & $1.12 \mathrm{E}+00$ & $1.13 E+00$ & $1.19 E-02$ & 6.3 & $\mathrm{Am} / \mathrm{Cm}$ \\
\hline & Am-243 & $<1.24 \mathrm{E}-02$ & $1.18 \mathrm{E}+00$ & $1.09 \mathrm{E}+00$ & $1.18 \mathrm{E}+00$ & $1.15 E+00$ & $5.35 E-02$ & 9.8 & Average \\
\hline & \multicolumn{6}{|c|}{ Radiological Constituents for Tank 6F Composite Sample \#2, $\mu \mathrm{Ci} / \mathrm{g}}$. & & & \\
\hline $295759-295761$ & Am-241 & $<3.14 \mathrm{E}-02$ & $6.71 \mathrm{E}+01$ & $6.44 \mathrm{E}+01$ & $6.08 \mathrm{E}+01$ & $6.41 \mathrm{E}+01$ & $3.16 E+00$ & 5 & Cs-removed \\
\hline \multirow[t]{2}{*}{$296594-296596$} & Am-241 & $6.62 \mathrm{E}-02$ & $6.71 \mathrm{E}+01$ & $7.61 \mathrm{E}+01$ & $6.40 \mathrm{E}+01$ & $6.91 \mathrm{E}+01$ & $6.31 E+00$ & 5 & $\mathrm{Am} / \mathrm{Cm}$ \\
\hline & Am-241 & $\leq 4.88 \mathrm{E}-02$ & $6.71 \mathrm{E}+01$ & $7.03 \mathrm{E}+01$ & $6.24 \mathrm{E}+01$ & $6.66 \mathrm{E}+01$ & $3.95 E+00$ & 5 & Average \\
\hline $295759-295761$ & Am-243 & $<2.19 \mathrm{E}-02$ & $1.52 \mathrm{E}+00$ & $1.35 \mathrm{E}+00$ & $1.36 \mathrm{E}+00$ & $1.41 \mathrm{E}+00$ & $9.52 E-02$ & 13.1 & Cs-removed \\
\hline \multirow[t]{3}{*}{$296594-296596$} & Am-243 & $<2.82 \mathrm{E}-03$ & $1.20 \mathrm{E}+00$ & $1.38 \mathrm{E}+00$ & $1.15 \mathrm{E}+00$ & $1.2 \mathrm{E}+00$ & $1.21 E-01$ & 6.3 & $\mathrm{Am} / \mathrm{Cm}$ \\
\hline & Am-243 & $<1.24 \mathrm{E}-02$ & $1.36 \mathrm{E}+00$ & $1.365 \mathrm{E}+00$ & $1.26 \mathrm{E}+00$ & $1.33 E+00$ & $6.21 E-02$ & 9.7 & Average \\
\hline & \multicolumn{7}{|c|}{ Radiological Constituents for Tank 6F Composite Sample \#3, $\mu \mathrm{Ci} / \mathrm{g}}$. & & \\
\hline $295762-295764$ & Am-241 & $<3.14 \mathrm{E}-02$ & $8.02 \mathrm{E}+01$ & $8.56 \mathrm{E}+01$ & $7.84 \mathrm{E}+01$ & $8.14 E+01$ & $3.75 E+00$ & 5 & Cs-removed \\
\hline \multirow[t]{2}{*}{$296597-296599$} & Am-241 & $6.62 \mathrm{E}-02$ & $8.74 \mathrm{E}+01$ & $8.78 \mathrm{E}+01$ & $8.24 \mathrm{E}+01$ & $8.59 \mathrm{E}+01$ & $3.00 E+00$ & 5 & $\mathrm{Am} / \mathrm{Cm}$ \\
\hline & Am-241 & $\leq 4.88 \mathrm{E}-02$ & $8.38 \mathrm{E}+01$ & $8.67 \mathrm{E}+01$ & $8.04 \mathrm{E}+01$ & $8.37 \mathrm{E}+01$ & $3.15 E+00$ & 5 & Average \\
\hline $295762-295764$ & Am-243 & $<2.19 \mathrm{E}-02$ & $1.88 \mathrm{E}+00$ & $1.77 \mathrm{E}+00$ & $1.88 \mathrm{E}+00$ & $1.85 \mathrm{E}+00$ & $6.12 E-02$ & 11.2 & Cs-removed \\
\hline \multirow[t]{2}{*}{$296597-296599$} & Am-243 & $<2.82 \mathrm{E}-03$ & $1.59 \mathrm{E}+00$ & $1.59 \mathrm{E}+00$ & $1.49 \mathrm{E}+00$ & $1.56 \mathrm{E}+00$ & $6.00 E-02$ & 6.5 & $\mathrm{Am} / \mathrm{Cm}$ \\
\hline & Am-243 & $<1.24 \mathrm{E}-02$ & $1.74 \mathrm{E}+00$ & $1.68 \mathrm{E}+00$ & $1.69 \mathrm{E}+00$ & $1.71 \mathrm{E}+00$ & $3.04 E-02$ & 8.9 & Average \\
\hline
\end{tabular}




\section{APPENDIX B - SUMMARY OF ANALYTICAL METHODS}

\section{Aqua Regia Digestions (AQR)}

Samples were digested according to procedure L16.1, ADS-2226. In a typical digestion, $\sim 0.5 \mathrm{~g}$ of Tank 6F composite sample was placed into a Teflon ${ }^{\circledR}$ digestion vessel. Then, $9 \mathrm{~mL}$ (hydrochloric acid) $\mathrm{HCl}$, and $3 \mathrm{~mL}$ (nitric acid) $\mathrm{HNO}_{3}$ were added to the Teflon ${ }^{\circledR}$ vessel. The Teflon $^{\circledR}$ vessel was sealed and heated for a period of no more than 4 hours at $115^{\circ} \mathrm{C}$. The sample was then cooled and diluted to $100 \mathrm{~mL}$. Three samples, in total, from each composite sample were digested by aqua regia.

\section{Sodium Peroxide/Hydroxide Fusions (PF)}

Samples were digested according to procedure L16.1, ADS 2502. In a typical digestion, $\sim 2$ grams of composite Tank 6F sample (as received) was placed into a nickel (Ni) crucible with a known weight. The remaining material in the crucible was fused at $675^{\circ} \mathrm{C}$ using a mixture of sodium peroxide (6.0 grams) and sodium hydroxide (4.0 grams). After the sample was cooled, water was added to dissolve the fused material and the solution was acidified by the addition of $25 \mathrm{~mL} \mathrm{HCl}$. The sample was diluted to $25 \mathrm{~mL}$. Three samples, in total, from each composite sample were digested by sodium peroxide fusion.

\section{Inductively Coupled Plasma - Atomic Emission Spectroscopy (ICP-ES)}

Samples are diluted as necessary to bring analytes within the instrument range. A scandium internal standard is added to all samples after dilution at a concentration of $2 \mathrm{mg} / \mathrm{L}$. The instrument is calibrated daily with a blank and two standards: 5 and $10 \mathrm{mg} / \mathrm{L}$ NIST traceable multi-element standards in dilute acid. Background and internal standard correction were applied to the results.

\section{Ion Chromatography for Anions (IC-Anions)}

For IC Anions, samples were diluted with a carbonate/bicarbonate diluent as necessary to bring analytes to within instrument calibration. A 3-point calibration curve is run daily on the instrument with concentrations of 10,25 and $50 \mu \mathrm{g} / \mathrm{mL}$.

\section{Atomic Absorption Spectroscopy (AA)}

Arsenic, selenium, and mercury are analyzed by AA. The mercury was determined using the cold vapor technique. Samples were diluted as necessary to bring analytes within the instrument calibration range. A 3-point calibration curve is run daily on the instrument with concentrations of $0.025,0.05$ and $0.075 \mathrm{mg} / \mathrm{L}$ for arsenic and mercury and $0.005,0.0075$, and $0.01 \mathrm{mg} / \mathrm{L}$ for selenium. An instrument blank is performed daily and a calibration check standard is run for each element at the beginning of the day, after each five sample runs, and at the end of the day. 


\section{Inductively Coupled Plasma - Mass Spectroscopy (ICP-MS)}

Samples were run concurrently with a laboratory control standard (LCS) containing V, Co, As, $\mathrm{Sr}, \mathrm{Mo}, \mathrm{Ru}, \mathrm{Ag}, \mathrm{Cd}, \mathrm{Sb}, \mathrm{Cs}, \mathrm{Ba}, \mathrm{La}, \mathrm{Eu}, \mathrm{Ho}, \mathrm{Yb}, \mathrm{Tl}, \mathrm{Pb}, \mathrm{Th}$, and $\mathrm{U}$. This LCS provided a mass response covering most of the mass range of interest. The following describes the calculation of the analytes of interest from the mass values:

Co $\quad$ mass 59

${ }^{99} \mathrm{Tc}$ mass 99 . Subject to interference when Ru is present in the sample.

Ag mass 107,109

$\mathrm{Pb} \quad$ mass 206, 207,208

Sn $\quad$ mass $117,118,120,122,124$

${ }^{233} \mathrm{U}$ mass 233

${ }^{234} \mathrm{U}$ mass 234

${ }^{235} \mathrm{U}$ mass 235

${ }^{236} \mathrm{U}$ mass 236

${ }^{238} \mathrm{U}$ mass 238

${ }^{237} \mathrm{~Np}$ mass 237

${ }^{239} \mathrm{Pu}$ mass 239

${ }^{240} \mathrm{Pu}$ mass 240

${ }^{242} \mathrm{Pu}$ mass 242.

\section{Gross Alpha/Gross Beta}

The solid material was too concentrated to be analyzed directly. Aliquots of peroxide fusion dissolution were added to liquid scintillation cocktail and analyzed for gross alpha and gross beta activity using liquid scintillation analysis. Alpha/beta spillover was determined for each aliquot analyzed, and subsequently used for accurately determining alpha and beta activity, via the addition of a known amount of plutonium to an identical aliquot of each sample.

\section{Other Specialty Separations and Preparations}

\section{Ni-59/63}

Aliquots of dissolution from the aqua regia digestion were aliquoted and spiked with an elemental nickel carrier. The nickel species were extracted from the matrix using dimethylglyoxime (DMG) based extractant. Ni-59 concentrations were measured using low energy photon/x-ray, thin-windowed, semi-planar high purity germanium spectrometers. Ni-63 concentrations were measured by liquid scintillation analysis. Elemental nickel carrier yields were measured by ICP-ES, and were used to correct the radioactive nickel species' analyses for any nickel losses from the radiochemical separations.

\section{Cs-137/Cs-134}

Aliquots of peroxide fusion dissolution and AQR were analyzed by coaxial high purity germanium gamma-ray spectrophotometers to measure Cs-137 and Cs-134.

\section{Sr-90}

Aliquots of peroxide fusion and $\mathrm{AQR}$ dissolutions were spiked with an elemental strontium carrier. The strontium species were extracted from the matrix using a crown-ether-based solid 
phase extractant. Sr-90 concentrations were measured by liquid scintillation analysis. Elemental strontium carrier yields were measured by neutron activation analysis, and were used to correct the $\mathrm{Sr}-90$ analyses for any strontium losses from the radiochemical separations.

\section{Pm-147/Sm-151}

Aliquots of peroxide fusion and AQR dissolution were spiked with an elemental samarium carrier. The promethium and samarium species were extracted from the matrix using a combination of Octylphenyl-N,N-di-isobutyl carbamoylphosphine oxide/tri-n-butyl phosphate (one CMPO/TBP) and di(2-ethylhexyl) orthophosphoric acid (one HDEHP based). Sm-151 and Pm-147 concentrations were measured by liquid scintillation analysis. The matrix was high in Sm-151, but the short-lived Pm-147 component of the material had decayed below noise levels of the analysis. Elemental samarium carrier yields were measured by neutron activation analysis, and were used to correct the analyses for any samarium losses from the radiochemical separations. The separation was designed to extract both $\mathrm{Sm}$ and Pm together; a Pm spike was run with the samples to confirm this.

\section{Co-60, Nb-94, Sb-126, Sn-126, Sb-126m, Eu-152, Eu-154, Eu-155, Am-241, Ac-227}

Aliquots of peroxide fusion were subjected to a Cs-removal process utilizing Bio Rad AMP-1 resin. The Cs-removed digestates were analyzed by coaxial high purity germanium spectrophotometers to measure the gamma-emitting radionuclides listed above.

\section{Pu-238, 239/240, 241}

Aliquots of peroxide fusion and AQR dissolutions were spiked with $\mathrm{Pu}-236$ tracer. The plutonium was extracted from the matrix using thenoyltrifluoroacetone (TTA) following a series of oxidation-state adjustments. The TTA extracts were mounted on stainless steel counting plates and counted for Pu-238 and Pu-239/240 using PIPs detectors. Each separation was traced based on the Pu-236 recovery. Aliquots of sample were also subjected to Cs-removal with Bio-Rad Ammonium Molybdophosphate (AMP) resin and extracted using TEVA columns (TEVA Brand name for one of Eichrom's resins). The Pu-containing extracts were measured by liquid scintillation analysis to determine $\mathrm{Pu}-241$ concentration.

\section{Am-241, 242m, 243, Cm-242, 243, 244, 245, 247, 248, Cf-249, 251, 252}

Samples of composite Tank $6 \mathrm{~F}$ materials were digested using a sodium peroxide fusion. Additionally, a matrix blank and matrix blank spiked with Am-241 and Cm-244 were prepared using Tank 8 simulated sludge. The americium, curium and californium species were extracted from aliquots of peroxide fusion using a CMPO/TBP based solid phase extractant and purified further with an HDEHP based solid phase extractant. Am-241, 243, Cm-243, 245, 247, Cf-249 and 251 concentrations were measured using low energy photon/x-ray, thin-windowed, semiplanar high purity germanium spectrometers. Am-241, Am-242m, Cm-242, 244, Cf-252 concentrations were measured using passivated, implanted, planar silicon (PIPS) alpha spectrometers. Some of the Am, Cm and Cf isotopes were also measured using ICP-MS. Am241 quantities had been measured from the gamma analyses, all $\mathrm{Am}, \mathrm{Cm}$, and $\mathrm{Cf}$ results were traced with the Am-241 present in the sample matrix. 


\section{U-232}

Aliquots of peroxide fusion were spiked with a U-233 radioactive tracer, additional aliquots were run through the method with no tracer added. Uranium was extracted from the matrix using two stages of a diamyl, amylphosphonate (DAAP)-based solid phase extraction and purified further via co-precipitation with cerium. U-232, U-233, and U-238 activities were measured using passivated, implanted, planar silicon (PIPS) alpha spectrometers. The Tank 6F composite samples were very high in U-234. Hence, U-233 tracers for U-234 (which have the same alpha energy as U-234) were swamped out. U-232/U-238 activity ratios were generated and were multiplied to U-238 activities measured with the ICP-MS to determine U-232 activities in the samples.

\section{Np-237}

Aliquots of peroxide fusion dissolution were traced with Np-239 (the daughter of Am-243 which was measured by Cs-removed gamma) and then purified with a quaternary amine based solid phase extraction. The purified aliquots were analyzed by low energy photon/x-ray, thinwindowed, semi-planar high purity germanium spectrometers to yield the $\mathrm{Np}-239$ recoveries and by the ICP-MS to measure Np-237. The Np-237 values were corrected with the decay-corrected $\mathrm{Np}-239$ recoveries.

\section{Tritium}

Aliquots of dissolution from the aqua regia digestion were subjected to tritium separations via steam distillation, and aliquots of the tritium-containing distillate were analyzed by liquid scintillation analysis.

\section{Se-79}

Samples of composite Tank 6F materials were weighed out, spiked with an elemental selenium carrier and digested in concentrated acid. The selenium species were extracted from the matrix using a combination of resin decontamination, selenium metal precipitation, and TBP-based liquid-liquid extractions. The purified selenium products were analyzed by liquid scintillation to measure Se-79, and by neutron activation analysis to measure elemental selenium carrier yields. The selenium carrier yields were used to correct the Se-79 analyses for any selenium losses from the radiochemical separations.

\section{Tc-99}

Tank 6F composite samples were digested in a combination of concentrated nitric and hydrochloric acids. Several matrix blanks were prepared using Tank 8 simulated sludge spiked with a Tc-99 standard. The dissolutions were subjected to a number of resin treatments to reduce dose prior to removal from the shielded cells. The treated samples were then spiked with Tc-99m and the technetium species were extracted from the matrix using an Aliquat-336 based solid phase extractant. Tc-99 concentrations were measured by liquid scintillation analysis. Tc-99m yields were measured with a NaI-well gamma spectrometer, and were used to correct the Tc-99 analyses for any technetium losses from the radiochemical separations. The average recovery of the Tc-99 spiked matrix blank was applied to the entire set of samples to correct for any losses from the decontamination steps used in the shielded cells. 


\section{Ra-226}

Tank 6F composite samples were digested using a sodium peroxide fusion. Each replicate was prepared in duplicate with the duplicate containing a Ra-224 tracer. Additionally, a matrix blank and matrix spiked blank were prepared using Tank 8 simulated sludge. The Ra-226 was extracted from the matrix using a combination of resin decontamination and ion exchange. The purified Ra-226 was sealed in polypropylene tubes and stored for several daughter Rn-222 halflives. The Ra-226 progeny daughter isotope $\mathrm{Pb}-214$ was then analyzed for using a high purity germanium well gamma ray spectrophotometer and results were corrected for the tracer Ra-224 recoveries.

\section{Pa-231}

Tank 6F composite samples were digested using a sodium peroxide fusion. Each replicate was prepared in duplicate with the duplicate containing a Pa-233 tracer. Additionally, a matrix blank and matrix spiked blank were prepared using Tank 8 simulated sludge. The dissolutions were decontaminated with AMP and quaternary amine based resins. Protactinium species were then extracted from the matrix using a CMPO/TBP based extractant. Pa-233 tracer concentrations were measured using high purity germanium spectrometers to determine separation yields. Pa231 was measured using the ICP-MS. The Pa-233 tracer yields were decay corrected and then used to correct the Pa-231 analyses for any losses from the radiochemical separations.

\section{I-129}

Tank 6F composite samples were dissolved in concentrated acid with an added KI carrier. A matrix blank and matrix blank containing an I-129 spike were also prepared using Tank 8 simulated sludge. Actinide and AMP resins were then added to the mixture to facilitate removal of interfering isotopes. Sodium sulfite is added to the material to reduce the iodine. Silver nitrate is added to the solution to precipitate the iodine as AgI, which is separated via filtration. The filtrate is analyzed for I-129 content using low energy photon/x-ray, thin-windowed, semiplanar, high purity germanium spectrometers. Elemental iodine yields were measured by neutron activation analysis, and were used to correct the I-129 analyses for any iodine losses from the radiochemical separation.

\section{C-14}

The solid Tank 6F composite material was used for the C-14 separation and analysis. The material was added to a mixture of sodium hydroxide, and sodium carbonate/sodium hydroxide. A series of oxidation and reduction steps designed to liberate $\mathrm{C}-14$ containing carbon dioxide were carried out, which selectively trapped the $\mathrm{C}-14$ in a basic solution. Finally, $\mathrm{C}-14$ containing carbon dioxide was captured in Carbosorb $\mathrm{E}$ and measured by liquid scintillation analysis. 


\section{Th-229/230, Ac-227}

Tank 6F composite samples were digested using a sodium peroxide fusion. Each replicate was prepared in duplicate with the duplicate containing a Th-229 tracer. Additionally, a matrix blank and matrix spiked blank were prepared using Tank 8 simulated sludge. The matrix spiked blank contained both a Th-228 and Th-229 spike. Thorium was extracted from the matrix using two stages of a quaternary amine based solid phase extraction and purified further via coprecipitation with cerium. Th-227, Th-229 and Th-230 concentrations were measured using passivated, implanted, planar silicon (PIPS) alpha spectrometers. The Th-229 tracer yields were used to correct the various analytes analyses for any thorium losses from the radiochemical separations. Ac-227 activities were calculated from the Th-227 results

\section{Nb-94}

Aliquots of peroxide fusion dissolution were spiked with $\mathrm{Nb}-95$ and then purified with an anion exchange. The purified aliquots were analyzed by high purity germanium spectrometers to measure $\mathrm{Nb}-94$ and to measure $\mathrm{Nb}-95$ tracer recoveries. The Nb-94 values were corrected with the stable $\mathrm{Nb}-95$ recoveries.

\section{Zr-93}

Zr was extracted from aliquots of peroxide fusion dissolution. Zr-93 levels were measured using the ICP-MS, and the results were yielded from sample stable $\mathrm{Zr}$ recoveries as measured by the ICP-MS.

\section{Cs-135}

Aliquots of dissolved material (alkali fusion digestion) were purified using a solvent-solvent caustic side solvent extraction-based (CSSX) extraction system. The purified Cs-containing aliquots were analyzed using ICP-MS to measure Cs-135/Cs-133 mass ratios. The Cs-133 and Ba-corrected Cs-135 ratios from the aliquots of separated material were used along with the associated Cs-133 ICP-MS result from the analysis of non-separated material to obtain a value for $\mathrm{Cs}-135$. The $\mathrm{Cs}-135$ result was then converted from $\mathrm{ug} / \mathrm{g}$ to $\mathrm{uCi} / \mathrm{g}$ using the specific activity of Cs-135.

\section{Weight Fraction Solids Measurement}

The weight percent total solids in each Tank 6F sample were measured in the Shielded Cells using a conventional drying oven at $110{ }^{\circ} \mathrm{C}$. An aliquot of each composite sample was placed in a container. The container was placed in the oven. The weights of the dried sample were checked periodically over 72 hours until two consecutive weights yielded comparable results. The weight fraction solid was calculated by dividing the dry weight of the sample by the initial weight of the sample. A 5\% sodium chloride salt solution prepared by dissolving 5 grams of dried sodium chloride in distilled water was used as the reference matrix for weight percent determinations as described above. 


\section{Density Measurement}

The bulk densities of the "as-received" granular Tank 6F solids were measured using a calibrated syringe tube assembly with graduation markings. With the syringe plunger removed, the syringe was loaded with Tank 6F solids with the help of a spatula to a level of about 2-mL. The plunger was then inserted into the syringe until the tip of the plunger touched the sample matrix, taking care not to excessively compress the sample. The plunger was then slightly pulled back and tapped to ensure the granular solid was uniformly distributed around the internal circumference of the tube. The volume of samples in the tube was read and the weight of the whole syringe assembly including the plunger was determined. A subtraction of the weight of the assembly without the granular samples provided the weight of the granular sample inside the syringe tube. Using the same syringe unit, the plunger was carefully removed and more granular sample was added to the syringe and the new volume and weight of the granular samples determined again. Using different syringe assemblies, this process was repeated three times for each Tank $6 \mathrm{~F}$ sample. 


\section{Appendix C Summary of Statistical Methods}

\subsection{INTRODUCTION}

Sampling has been completed for the characterization of the residual material on the floor of Tank 6F in the F-Area Tank Farm at the Savannah River Site (SRS), near Aiken, SC. The sampling was performed by Savannah River Remediation LLC (SRR) using a stratified random sampling plan with volume-proportional compositing, Broaden ${ }^{12}$. The plan consisted of partitioning the residual material on the floor of Tank $6 \mathrm{~F}$ into three non-overlapping strata: an area of accumulation under Riser 5, a North Area, and a South Area.

Each of three composite samples was constructed from five primary samples of material. A total of 15 primary samples were planned, but one sample, 6-B3a, could not be obtained. The replacement material for the unavailable sample was described by Martin ${ }^{14}$. The volume of residual material in each of the three strata was estimated along with an uncertainty of the volume estimate. A separate set of strata volumes was randomly generated for each composite sample from the volume uncertainty distributions, Dean ${ }^{1}$. All three strata volumes were converted into volumetric proportions, and subsequently to the mass of residual material to be used from each primary sample for its corresponding composite sample. This procedure directly incorporates the volume uncertainty into the variation among the analyte concentrations in the composite samples, Shine ${ }^{16}$. Each composite sample was measured three times for all but a few radionuclide concentrations.

A remote-controlled crawler was designed to collect the sample material. Tank $6 \mathrm{~F}$ contains an extensive network of cooling coils that fettered movement of the crawler across the tank floor. Consequently, sample locations within strata were selected based on their accessibility to the crawler. However, it is assumed that earlier stages of cleaning/mixing of the material in the tank raised particles off the tank floor, and then deposited them in random locations, so the sample collection was effectively based on random sampling.

\subsection{OBJECTIVE AND SCOPE}

The objective of this appendix is to perform a statistical analysis of the chemical and isotopic concentration results for the residual material on the floor of Tank $6 \mathrm{~F}$. The approach is to use samples representative of the material from the tank floor to estimate the concentrations of analytes in the remaining residual material. The concentration results are summarized by the means and standard deviations of the composite sample concentrations. Upper 95\% confidence limits (UCL95s) are calculated for the actual mean concentration of each analyte.

The statistical analyses are applied to a subset of the measured analytes. Table 16 from SRR ${ }^{13}$ lists the analytes to be considered in these analyses. 
Table 16 Tank 6F Constituents for Statistical Evaluation

\begin{tabular}{|c|c|c|c|c|}
\hline Density & $\mathrm{Eu}-152$ & Sb-126 & $\mathrm{Ag}$ & $\mathrm{Mn}$ \\
\hline Ac- 227 & Eu-154 & $\mathrm{Sb}-126 \mathrm{~m}$ & $\mathrm{Al}$ & Mo \\
\hline Am-241 & H-3 & Se-79 & As & $\mathrm{Ni}$ \\
\hline Am-242m & I-129 & Sm-151 & $\mathrm{B}$ & $\mathrm{NO}_{2}^{-1}$ \\
\hline Am-243 & $\mathrm{Nb}-94$ & Sn-126 & $\mathrm{Ba}$ & $\mathrm{NO}_{3}^{-1}$ \\
\hline Ba-137m & $\mathrm{Ni}-59$ & Sr-90 & $\mathrm{Cd}$ & $\mathrm{Pb}$ \\
\hline C-14 & $\mathrm{Ni}-63$ & Tc-99 & $\mathrm{Cl}^{-1}$ & $\mathrm{PO}_{4}{ }^{-3}$ \\
\hline Cf-249 & Np-237 & Th-229 & $\mathrm{Co}$ & $\mathrm{Sb}$ \\
\hline Cm-243 & Pa-231 & Th-230 & $\mathrm{Cr}$ & $\mathrm{Se}$ \\
\hline $\mathrm{Cm}-244$ & $\mathrm{Pu}-238$ & U-232 & $\mathrm{Cu}$ & $\mathrm{SO}_{4}{ }^{-2}$ \\
\hline $\mathrm{Cm}-245$ & $\mathrm{Pu}-239$ & U-233 & $\mathrm{F}$ & $\mathrm{Sr}$ \\
\hline Cm-247 & $\mathrm{Pu}-240$ & U-234 & $\mathrm{Fe}$ & $\mathrm{U}$ \\
\hline Cm-248 & $\mathrm{Pu}-241$ & U-235 & $\mathrm{Hg}$ & $\mathrm{Zn}$ \\
\hline Co-60 & $\mathrm{Pu}-242$ & U-236 & $\mathrm{I}$ & $\mathrm{Wt} \%$ Solids \\
\hline Cs-135 & $\mathrm{Pu}-244$ & U-238 & & \\
\hline \multirow[t]{2}{*}{ Cs-137 } & Ra-226 & $\mathrm{Y}-90$ & & \\
\hline & & Zr-93 & & \\
\hline
\end{tabular}

\subsection{STATISTICAL METHODS}

The material in each composite sample is considered to be representative of all of the residual material on the floor of Tank $6 \mathrm{~F}$, and thus the measured concentration for any analyte in a composite sample is considered to be an independent estimate of the actual mean concentration of the analyte in the residual material on the entire tank floor. Three concentration measurements were performed for each analyte on each composite sample. The statistical measurement error model for a concentration measurement result $Y_{i j}$ is

$$
Y_{i j}=\mu+s_{i}+\varepsilon_{i j},
$$

where $Y_{i j}$ is the $j$-th measured concentration for an analyte in composite sample $i, \mu$ is the actual mean analyte concentration for all of the residual material on the floor of Tank $6 \mathrm{~F}, s_{i}$, the sampling error, is a random effect, the difference between the actual mean concentration in composite sample $i$ and the actual mean concentration for all of the residual material on the tank floor that arises from heterogeneity, sampling, sample preparation, and volumetric proportion errors, and $\varepsilon_{i j}$, distributed with mean zero and standard deviation $\sigma$, is the difference between concentration measurement $j$ on sample $i$ and the actual mean concentration in composite sample $i, i=1,2,3 ; j=1,2,3$.

A test for heterogeneity of measurement variance was performed prior to other analyses in order to verify the assumption that the composite sample material is well-mixed and the measurement variance $\sigma^{2}$ is the same for all composite samples. The test procedure is the Levene's test with a Type I family-wise error rate $\alpha=0.05$. Since the sample sizes are small (no more than three 
measurement results per composite sample), a Bonferroni procedure is used to control for spuriously significant results by dividing the 0.05 family-wise ${ }^{1}$ error rate by the number of comparisons to obtain the Type I error rate per comparison. The Bonferroni criteria for individual analyte test are $\alpha=0.05 / 5=0.0100$ for anions, $\alpha=0.05 / 18=0.0028$ for inorganics, and $\alpha=0.05 / 36=0.0014$ for radionuclides. If the P-value for an individual constituent test is less than the Bonferroni $\alpha$, then it is concluded that the laboratory variances are not the same for all of the composite samples.

An analysis of variance (ANOVA) F test was performed in order to determine whether the random effect $s_{i}$ is warranted in Eqn (1). If the F test results indicate a statistically significant sampling error $s_{i}$ at a level of significance $\alpha=0.05$, then Eqn (1) becomes the basis for estimating the true mean concentration in the residual material; if the ANOVA F test result is not statistically significant, then the random effect $s_{i}$ is not needed and Eqn (1) reduces to the following:

$$
Y_{i j}=\mu+\varepsilon_{i j}
$$

where there is no sampling error term $s_{i}$ in the model.

If all of the concentration measurements for an analyte are above detection, then the ANOVA F test can be performed, and a decision made to use the model in Eqn (1) with the random effect if $F \geq F_{0.95,2,6}=5.14325$, and to use the model in Eqn (2) without the random effect if $F<F_{0.95,2,6}=5.14325$. When $F \geq F_{0.95,2,6}=5.14325$, the UCL95 for the actual mean tank concentration is given by

$$
U C L_{95 \%}=\bar{Y}+t_{0.95,2 d f} \cdot \sqrt{\frac{M S_{\text {Sample }}}{9}}
$$

where $\bar{Y}_{.}$is the sample mean concentration of the nine concentration measurement results, and $M S_{\text {sample }}$ is the estimate of the mean square for the random effect $s_{i}$ in the model in Eqn (1), where

$$
M S_{\text {sample }}=\frac{\sum_{i=1}^{3} \frac{Y_{i \cdot}^{2}}{3}-\frac{Y_{.}^{2}}{9}}{6},
$$

and $Y_{i}$ and $Y_{.}$are the total of the three measured concentration results for composite sample $i, i$ $=1,2,3$ and the total of the nine measured concentration results for all three composite samples.

\footnotetext{
${ }^{1}$ A family-wise error rate refers to the error rate of making at least one Type I error (rejecting the null hypothesis when it is true) in a prescribed family or set of tests, where family refers to all analytes in the set of all inorganic constituents, the set of all radionuclides, or the set of all anions. Controlling the family-wise error rate means that the probability of making at least one Type I error for individual analytes in a family will be no more than a stated $\alpha$ probability.
} 
The estimated standard error of the mean concentration is the square root of the $\left(M S_{\text {Sample }} / 9\right)$ when all composite samples have three measurements. Tables 21 and 22 provides a more general formula for the estimated standard error of the mean when the composite samples have been measured different numbers of times.

When $F<F_{0.95,2,6}=5.14325$, the UCL95 for the actual mean tank concentration is given by

$$
U C L_{95 \%}=\bar{Y}+t_{0.95,9-1 d f} \cdot \sqrt{\frac{s^{2}}{9}}
$$

where $s$ is the sample standard deviation of all nine measured concentration results.

The above procedures are appropriate if the data or a transform of the data approximately follow the normal distribution. Figure 3 presents a sequence of goodness-of-fit tests to identify a distribution consistent with the measurement results and select an estimation method for the mean, standard deviation, and UCL95. Studies by Singh, Singh, and Englehardt ${ }^{17}$ demonstrated that using the coefficient of variation (the percent standard deviation) is much less effective than using a formal goodness-of-fit test to determine whether the concentration measurements are consistent with a particular distribution such as the normal distribution. Consequently, the normal distribution assumption is tested by the Wilk-Shapiro (W-S) goodness-of-fit test at an $\alpha$ $=5 \%$ level of significance. If the $\mathrm{W}-\mathrm{S}$ statistic is less than the $\mathrm{W}-\mathrm{S}$ critical value, then normality is rejected; if there is no statistically significant departure from normality, the mean, standard deviation, and UCL95 are estimated based on a normal distribution.

If the normal distribution assumption is rejected by the $\mathrm{W}-\mathrm{S}$ test, then the measurements are tested to determine whether they are consistent with a skewed distribution. This report adopts the strategy in Singh, Armbya, and $\operatorname{Singh}^{18}$ to test for the gamma distribution prior to the lognormal distribution. The gamma distribution assumption is tested using Anderson-Darling (A-D) goodness-of-fit statistic. If the A-D statistic exceeds the A-D critical value then the gamma distribution assumption is rejected; if there is no statistically significant departure from the gamma distribution, the mean, standard deviation, and UCL95 are determined based on a gamma distribution. If the gamma distribution is rejected, but a plot of the concentration results versus the theoretical gamma quantiles displays a linear pattern with high correlation (over 95\%), then the results are said to follow an approximate gamma distribution. The mean, standard deviation, and UCL95 are estimated assuming a gamma distribution, according to Singh, Armbya, and Singh ${ }^{18}$.

Finally, if the gamma distribution is rejected and the gamma quantile plot does not exhibit high correlation ( $>95 \%$ ), then the W-S goodness-of-fit test is used to determine if the measurements are consistent with the lognormal distribution. If the W-S statistic is less than the W-S critical value, then the lognormal assumption is vacated and a nonparametric approach to estimation is adopted; if the W-S test determines that the lognormal distribution is plausible, then the lognormal distribution is adopted. Appropriate UCL95s based on the lognormal distribution and the nonparametric Chebyshev UCL95 for use when the lognormal distribution is rejected are documented by Singh, Singh, and Englehardt ${ }^{17}$. 


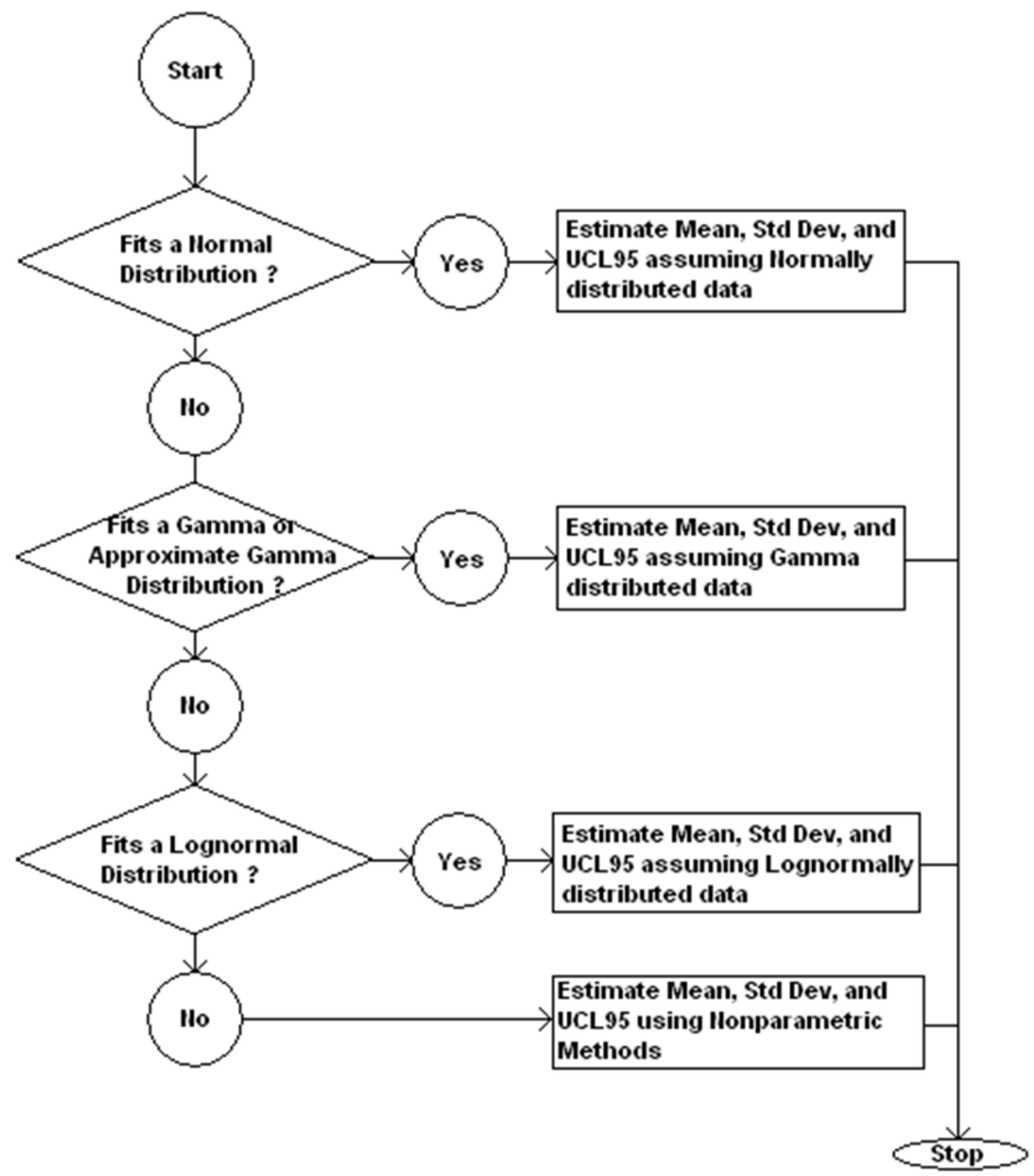

Figure 3. Sequence of Goodness-of-Fit Tests to Identify a Distribution and Select an Estimation Method

Heterogeneity and ANOVA tests were performed in SAS JMP® 9.0.0 software from SAS Institute, Inc. ${ }^{15}$, and distribution plotting, goodness-of-fit tests, and parameter estimation were performed in ProUCL 4.1.01 ${ }^{18}$ software developed by Singh, Armbya, and Singh ${ }^{18}$. P-values for the Wilk-Shapiro goodness-of-fit tests were obtained from SAS JMP® 9.0.0 software. Software validation and verification for SAS JMP® 9.0.0 and ProUCL 4.1.01 are documented by Baker and Others ${ }^{11}$.

\subsection{STATISTACAL ANALYSIS}

The statistical analyses are based on the measurement results presented in Tables 7, 8, and 9 for anions, Tables 10, 11, and 12 for inorganic constituents, and in Tables 13, 14, and 15 for 
radionuclides. Composite Sample Bulk Densities $(\mathrm{g} / \mathrm{mL})$ were obtained from Table 4 and Weight Percent Solids ( $\mathrm{Wt} \%$ ) were obtained from Table 6. The following subsections describe the application of the statistical methods described in Section 3.0. None of these measurement results were below their minimum detectable concentrations (MDCs).

\subsection{Analysis of Density, Weight Percent Solids, and Anion Concentrations}

Density, Weight Percent Solids, and six anions were included in the key list of constituents for statistical analyses. Detailed tables are presented in Appendix C Table 17.

Results for anions Cl-1, F, and PO4-3 were below their MDCs. The minimum and maximum MDCs for each of these analytes is reported in Table 18.

Levene's test for heterogeneity of variance was applied to the density, weight percent solids, and anion results with family-wise $\alpha=0.05$. Referring to Table 17, the Levene's test is not statistically significant (P-value $>\alpha=0.0100$ ) for density, weight percent solids, or any anion concentration. Therefore, the tests to determine whether there is variance among the composite samples will be performed using an ANOVA (which assumes a constant measurement error variance). The ANOVA F-test was statistically significant (Pvalue $<\alpha=0.05$ ) for density, weight percent solids, and NO2-1. Consequently, the model in Eqn (1) with the sampling error was adopted for those measurements. Their UCL95s are based on the one-sided upper Student's $\mathrm{t}$ confidence interval. The ANOVA F-Test was not statistically significant for NO3-1 and SO42. ProUCL 4.1 software was used to determine an appropriate distribution and UCL95 NO3-1 and SO4-2. Table 19 summarizes the results for the UCL95s.

\subsection{Analysis of Inorganic Consituents}

The inorganic constituents that are analyzed in this report are listed in Table 16. Figure 4 displays scatterplots of all pairs of inorganic constituents with Composite Samples 1, 2, and 3 shown as red, blue, and black circles, respectively. The three replicate measurements for each composite sample are identified by their run number $(1,2$, or 3$)$ in Figure 4 . An overall picture emerges across all inorganic constituents that Composite Sample 1 tends to exhibit the lowest concentrations, Composite Sample 3 tends to show the highest concentrations, while Composite Sample 2 tends to have intermediate concentrations.

In Section 7.2 Statistical Tables for the Inorganic Constituents, Levene's test for heterogeneity of variance was applied to each inorganic constituent with $\alpha=0.0028$. Based on Bonferroni's procedure, $\mathrm{Alt}^{10}$, this yields a tolerance of no more than 0.05 for the probability of at least one Type I error. The P-values for Levene's test are given in Table 20 None of these constituents have a statistically significant test result for heterogeneity of variance. Therefore, the following test to determine whether there is a variance among the composite samples will be performed using an ANOVA (which assumes a constant measurement error variance). 
Each inorganic constituent was also subjected to an ANOVA test to determine if a sampling variance should be included in the model. As seen by the ANOVA P-values in Table 20, all inorganic constituents except $\mathrm{Hg}$ and Mo have statistically significant sampling variances.

The only potential outliers in the set of inorganic measurements appear to be one $\mathrm{Hg}$ measurement on Run 1 of Composite Sample 1 and one Mo measurement on Run 1 of Composite Sample 3. They do not align along the linear path traced by the other measurements in Figure 4 plots. The other two Hg measurements on Composite Sample 1 are consistent with all of the other $\mathrm{Hg}$ measurements on Composite Samples 2 and 3, and the other two Mo measurements on Composite Sample 3 are consistent with all of the other Mo measurements on composite Samples 1 and 2. The magnitudes of the differences among the $\mathrm{Hg}$ and $\mathrm{MO}$ results may not have practical significance. No numerical correlations are shown since there are only three composite samples.

Removing both of these potential outliers from the database, reduces the variability and results in statistically significant sampling variances, as was the case for all other inorganics. The computations for UCL95s for $\mathrm{Hg}$ and Mo when the potential outiers are omitted are detailed in Tables 21 and 22, respectively. . It is recommended to remove the $\mathrm{Hg}$ and Mo outliers. Doing so results in more conservative (larger) UCL95s, and all inorganic results with measurements above their MDCs exhibit heterogeneity.

Several inorganics, $\mathrm{Ag}, \mathrm{As}, \mathrm{B}, \mathrm{Sb}$, and $\mathrm{Se}$, had all measurements reported below their MDCs. Their minimum and maximum MDCs are given in Table 23. Table 24 provides a listing of UCL95s for the inorganics with results above their MDCs. The UCL95 results for Hg and Mo with and without their potential outliers are given in Table 24. It is recommended that the results without the potential outliers be used, since (1) those results are about the same or more conservative (higher UCLs) than those with all of the data and (2) the sampling error model obtained without the potential outliers is consistent with all of the other inorganics. 


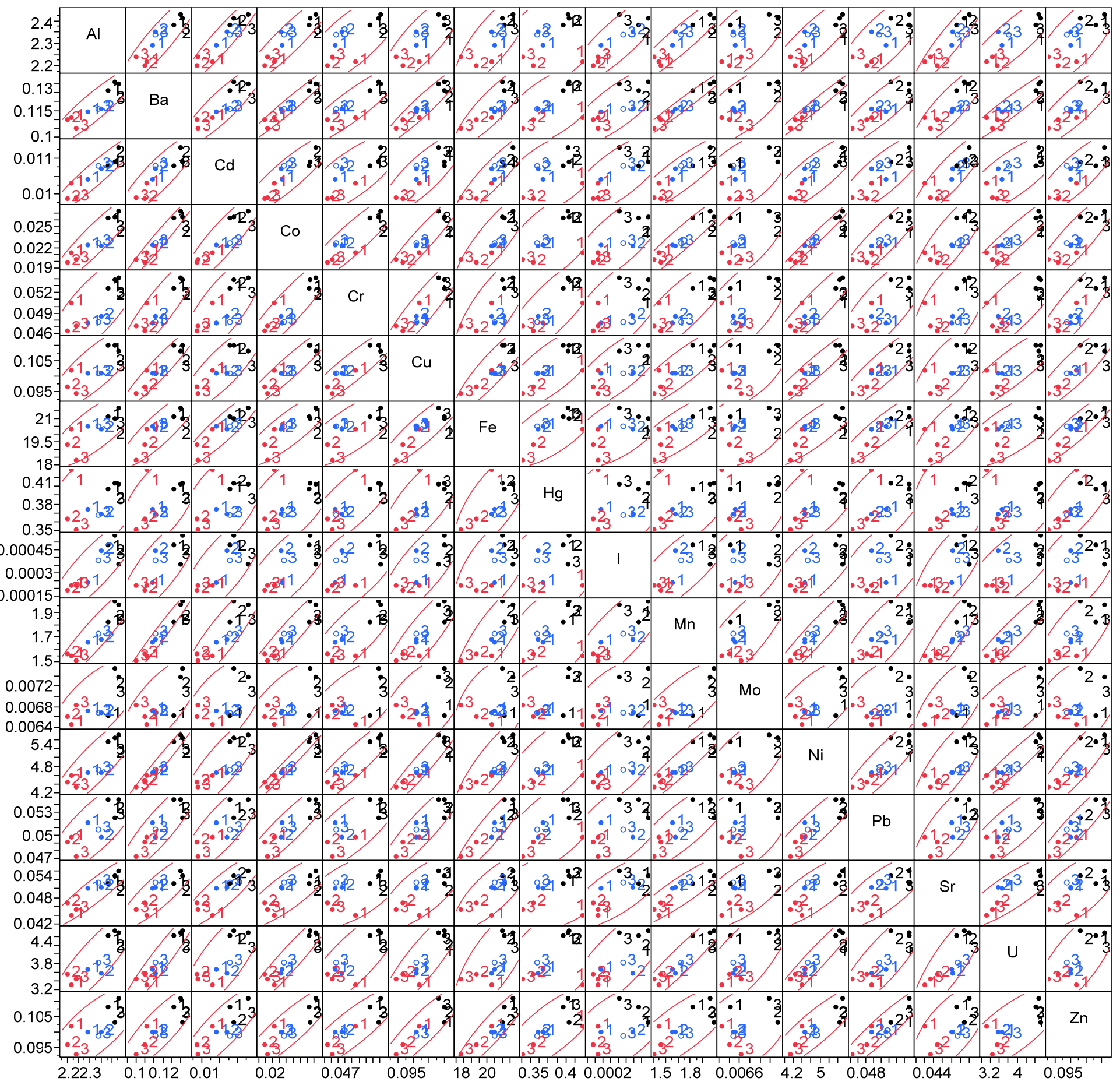

Figure 4. Scatterplots for the Inorganic Constituents

(Legend: Composite Samples 1 = red, 2 = blue, and 3 =black) 


\subsection{Analysis of Radionuclides}

The radionuclides that are analyzed in this report are listed in Table 16 Figure 5 displays scatterplots of a subset of all pairs of radionuclides since there were too many to effectively plot. Composite Samples 1, 2, and 3 are shown as red, blue, and black circles, respectively. The three replicate measurements for each composite sample are not uniquely identified in Figure 5. Sample 3 has the highest measured concentrations of radionculides as well as for inorganic constituents. There is no consistent pattern of higher and lower concentrations for Composite Samples 1 and 2 as was seen with the inorganic constituents.

In Table 25, Levene's test for heterogeneity of variance was applied to each radionculide with an $\alpha=0.0014$ for each comparison. Using the Bonferroni's approach to multiple comparisons in $\mathrm{Alt}^{10}$, this yields a tolerance of no more than 0.05 for the probability of at least one Type I error among the radionuclides. The P-values for Levene's test are given in Table 25 for each of the radionculides. None of these radionculides has a statistically significant test result for heterogeneity of variance. Therefore, each radionuclide was also subjected to an ANOVA test to determine if a sampling variance should be included in the model. As seen by the ANOVA Pvalues in Table 25, only ten radionuclides have statistically significant sampling variances.

Tweleve radionuclides had all measurements below their MDCs. The minimum and maximum MDCs for each of these radionuclides is given in Table 26. The estimated mean, estimated standard error of the mean, and the UCL95 for all radionuclides with measurements above their MDCs is given in Table 27. The UCL95s for those radionuclides that had the No Sampling Error model type were computed in ProUCL 4.1 software. Table 27 lists the resulting distribution and values for the UCLs. 


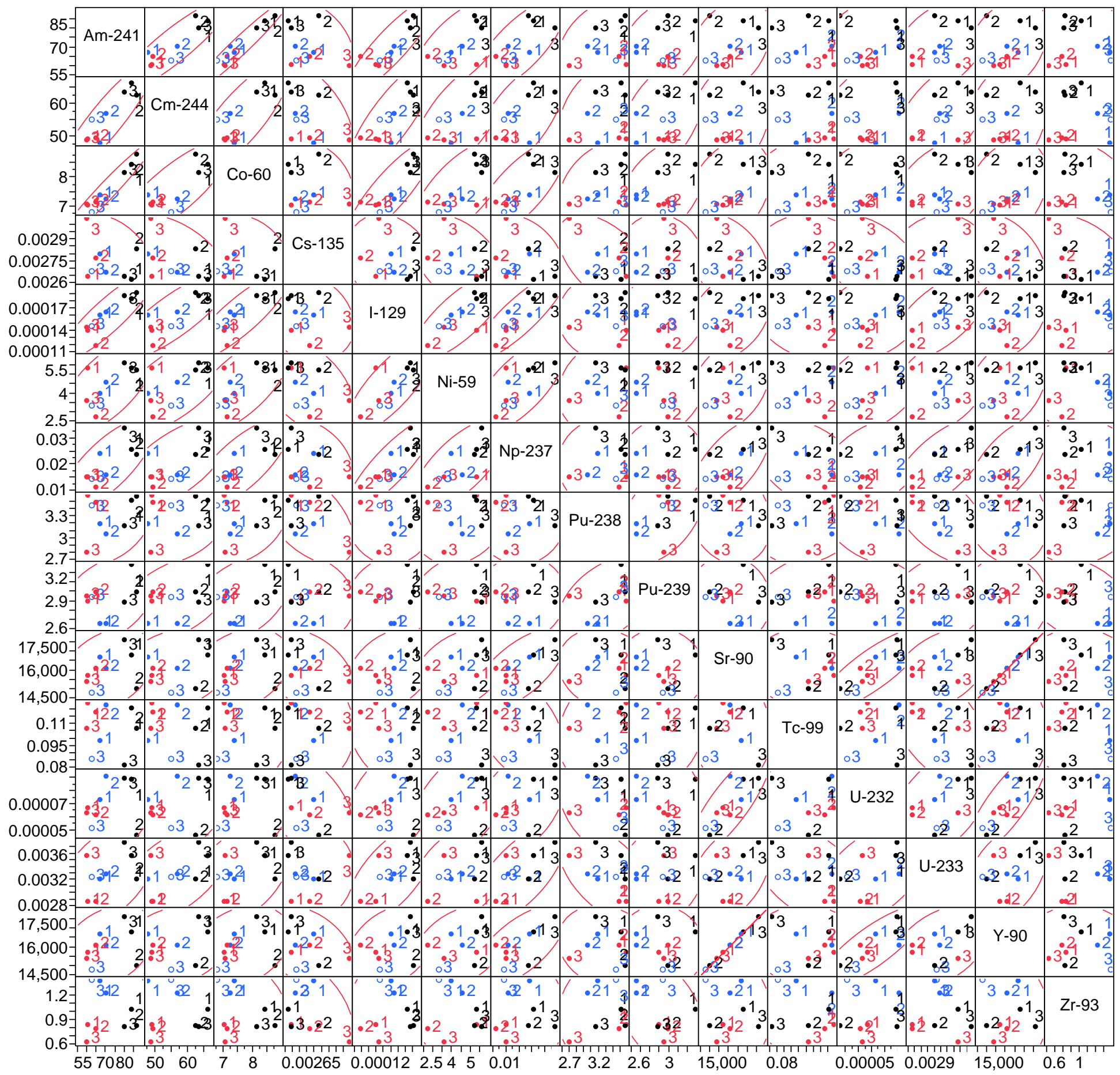

Figure 5. Scatterplots for a Subset of the Radionuclides

(Legend: Composite Samples 1 = red, 2 = blue, and 3 =black) 


\subsection{CONCLUSIONS AND RECOMMENDATIONS FOR THE STATISTICAL APPENDIX}

This appendix documents the statistical summary of the Tank $6 \mathrm{~F}$ floor composite sample results.

The estimated mean concentration, the estimated standard error of the mean concentration, and the UCL95 for the bulk densities, the weight percent solids, and the anion concentrations with measurements above their MDCs are summarized in Table 19. Those anions without results above their MDCs were listed in Table 18 along with their minimum and maximum MDCs.

All of the inorganic constituents displayed heterogeneity as evidenced by the ANOVA results. The inorganic results demonstrated consistent differences across the composite samples: lowest concentrations for Composite Sample 1, intermediate-valued concentrations for Composite Sample 2, and highest concentrations for Composite Sample 3. The Hg and Mo results suggest possible measurement outliers. However, the magnitude of the difference between the $\mathrm{Hg}$ UCL95 results with and without the Hg outlier and the magnitude of the difference between the Mo UCL95 results with and without the Mo outlier do not appear to have practical significance. It is recommended to remove the potential measurement outliers. Omitting the outliers is conservative in the sense of producing a higher UCL95 for Hg and Mo than if the potential outliers were included in the calculations. The estimated mean, estimated standard deviation, and the UCL95 for each inorganic constituent are summarized in Table 24.

In contrast to the inorganic results, most of the radionuclides did not demonstrate heterogeneity among the composite sample results. The estimated mean concentrations, the estimated standard errors of the mean concentrations, and the UCL95s for the radionuclide mean concentrations are summarized in Table 27.

\subsection{REFERENCES FOR THE STATISTICAL APPENDIX}

10. [1982] Alt, F.B. "Bonferroni Inequalities and Intervals," in Kotz, S. and Johnson, N.L. Encyclopedia of Statistical Sciences, John Wiley and Sons, NY.

11. [2012] Baker, R.A.; Edwards, T.B.; Harris, S.P.; Harvel, C.D.; and Shine, E.P. "Verification and Validation for Commercial Statistical Packages Utilized by Applied Computational engineering and Statistics," B-VVR-A-00002, Revision 1 (May).

12. [2011] Broaden, D.A. "Tank 6F Sampling and Analysis Plan," Savannah River Remediation LLC SRR-LWE-2010-00300, Revision 1 (January 5).

13. [2012] SRR. "Tanks 5 and 6 - TTR, Rev. 7 (June 7, 2012)

14. [2011] Martin, B.A. Justification for Adjustments to the Tank 6F Sample Plan, Revision 0, SRR-CWDA-2011-00159, Aiken, SC, October 2011.

15. [2010] SAS Institute, Inc. Basic Analysis and Graphing, JMP 9.0.0 On-line Help Books, Cary, NC.

16. [2011] Shine, E.P. "Technical Review of the Method of Constructing Composite Samples with Uncertain Volumetric Proportions," SRNL-STI-2011-00323, May 24. 
17. [1997] Singh, A.K.; Singh, A; and Englehardt, M. "The Lognormal Distribution in Environmental Applications,” EPA/600/S-97/006 (December).

18. [2010] Singh, A.; Armbya, N.; and Singh, A.K. "ProUCL Version 4.100 Technical Guide (Draft)" US EPA/600/R-07/041 (May).

\subsection{SUPPORTING STATISTICAL TABLES}

The statistical tables supporting the results discussed in Section 4 are presented in Section 7. 
APPENDIX C: STATISTICAL RESULTS FOR ANIONS

Table 17 Tests and Estimators for Density, Weight Percent, and Anions

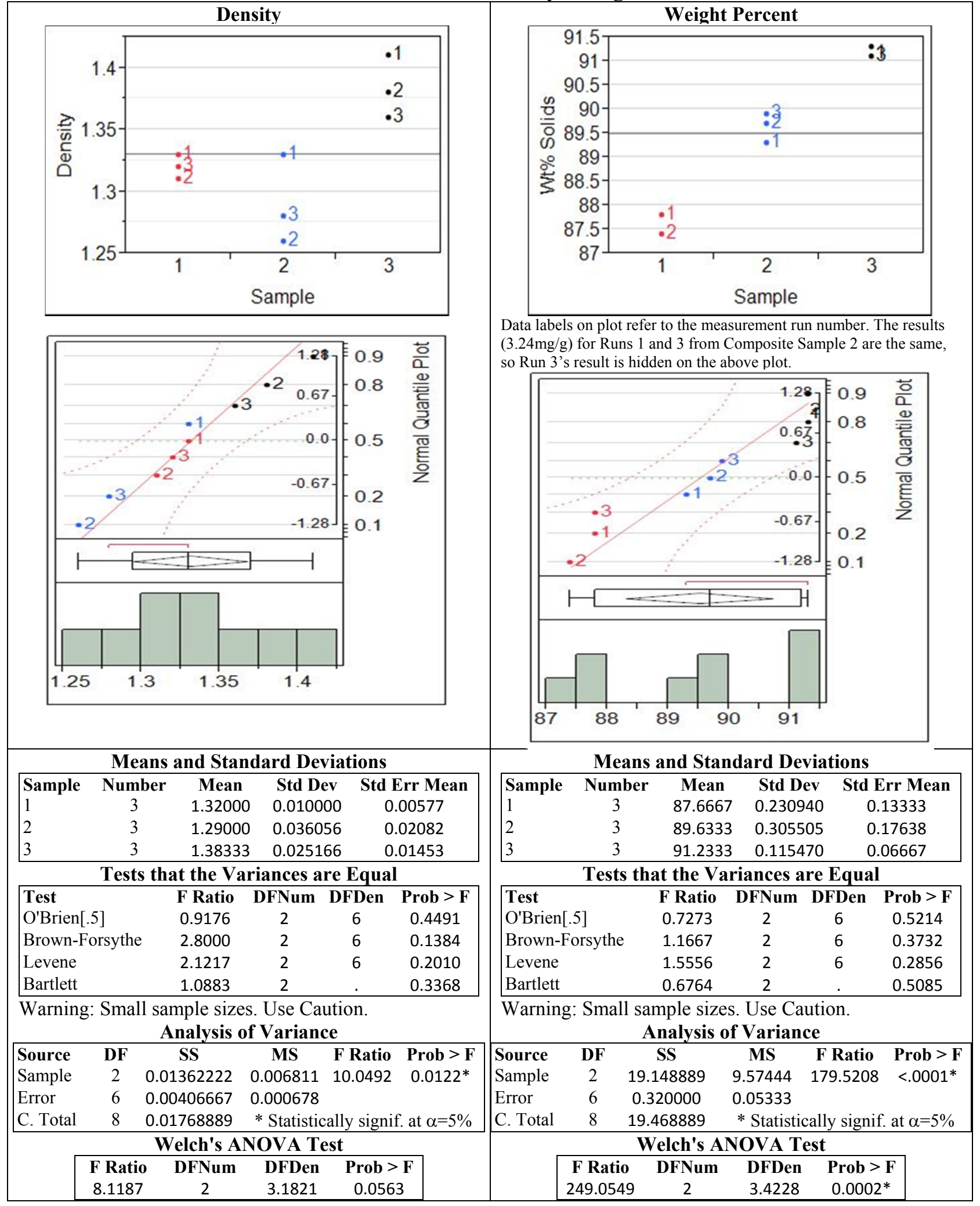


APPENDIX C: STATISTICAL RESULTS FOR ANIONS

Table 17 Tests and Estimators for Density, Weight Percent, and Anions

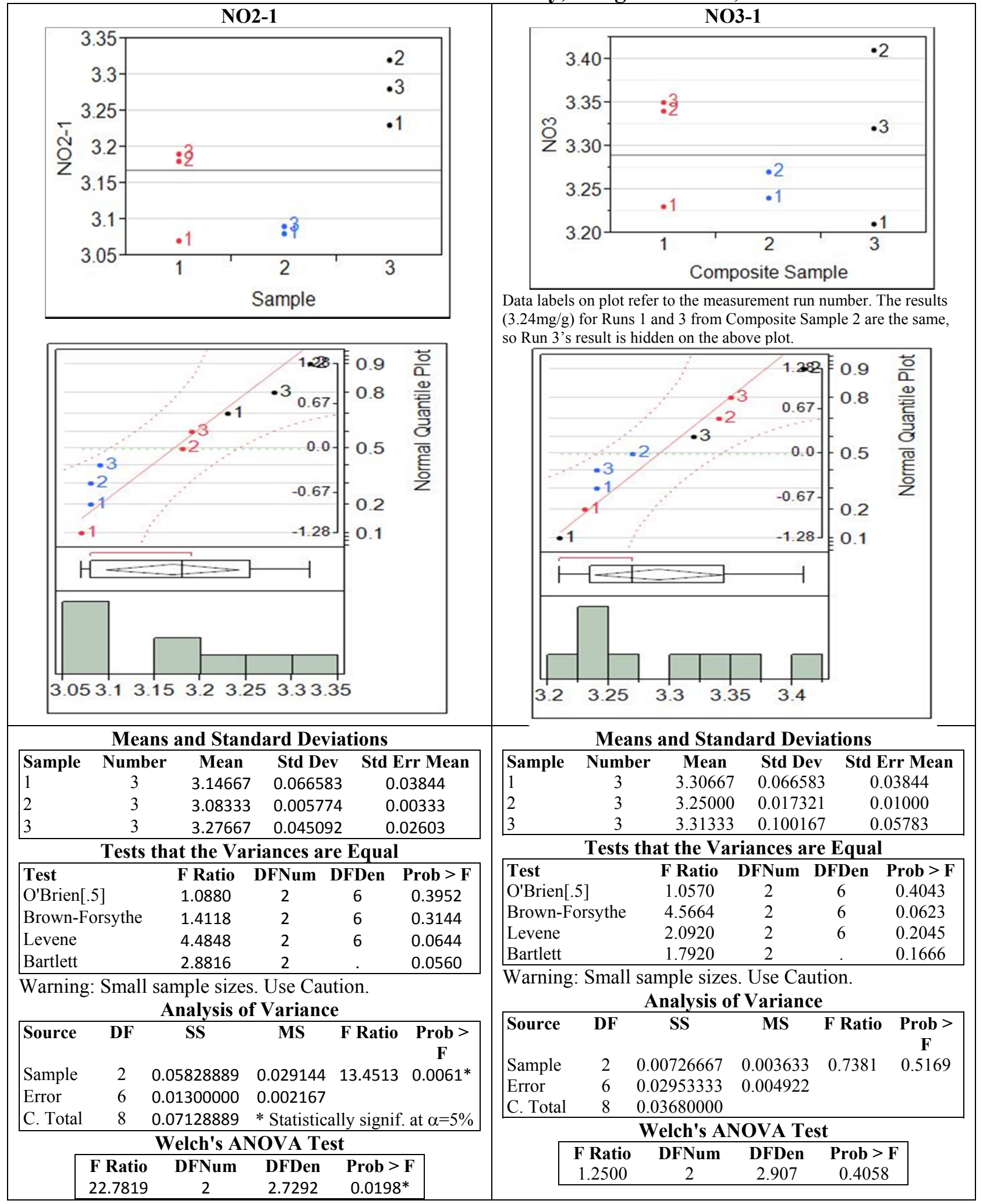


Table 17 Tests and Estimators for Density, Weight Percent, and Anions

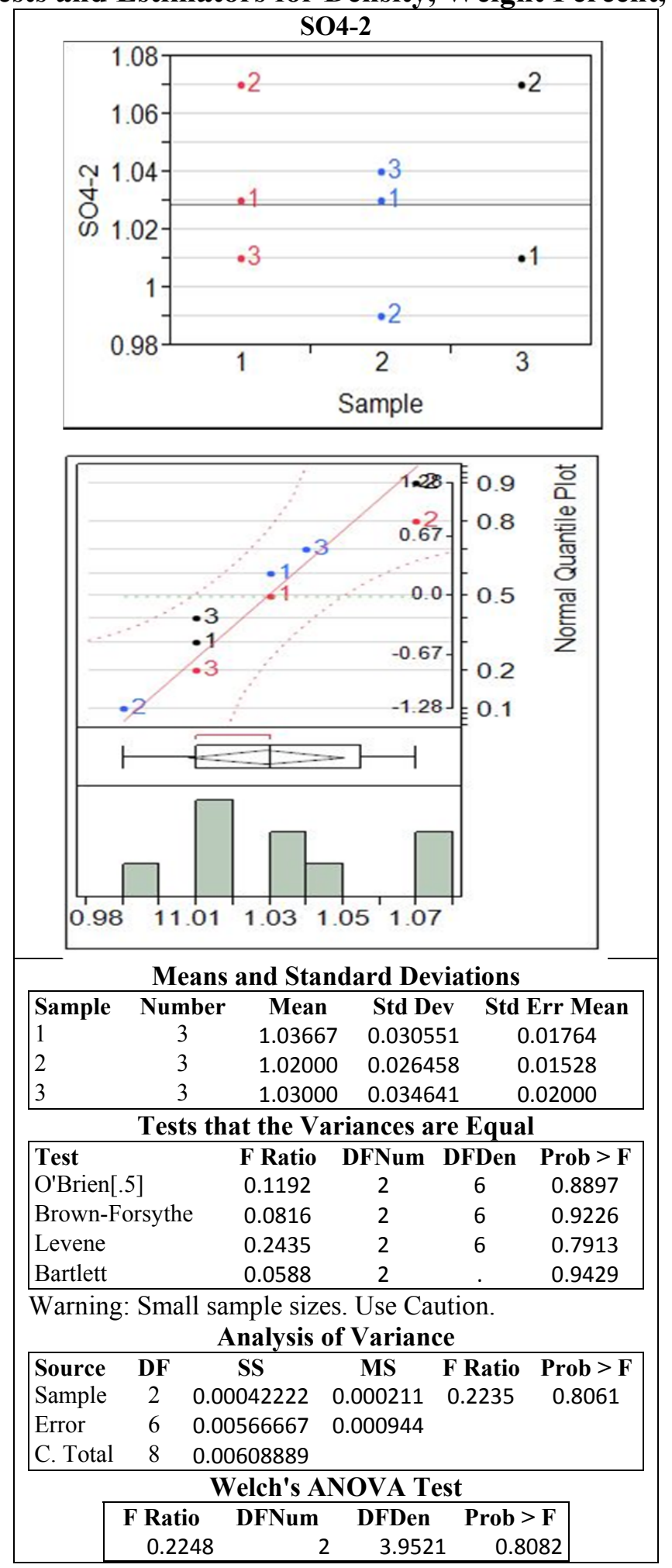


APPENDIX C: STATISTICAL RESULTS FOR DENSITY, WEIGHT PERCENT, AND ANIONS

Table 18 Anion and Tritium Concentrations less than their MDCs

\begin{tabular}{|l|c|c|}
\hline & \multicolumn{2}{|c|}{ MDCs } \\
\hline & Min & Max \\
\hline Cl-1 & $4.00 \mathrm{E}-02$ & $4.00 \mathrm{E}-02$ \\
\hline F-1 & $4.00 \mathrm{E}-02$ & $4.00 \mathrm{E}-02$ \\
\hline PO4-3 & $4.00 \mathrm{E}-02$ & $4.00 \mathrm{E}-02$ \\
\hline
\end{tabular}

Table 19 95\% UCLs for Mean Density, Weight Percent Solids, and Anion Concentrations (mg/g)

\begin{tabular}{|c|l|c|c|c|c|l|}
\hline Anion & \multicolumn{1}{|c|}{ Model Type } & N & Mean & $\begin{array}{c}\text { Standard } \\
\text { Deviation }\end{array}$ & UCL95 & \multicolumn{1}{|c|}{ Notes } \\
\hline Density & Sampling Error & 9 & $1.331 \mathrm{e}+0$ & $5.217 \mathrm{e}-2$ & $1.411 \mathrm{e}+0$ & Normal \\
\hline $\begin{array}{c}\text { Weight \% } \\
\text { Solids }\end{array}$ & Sampling Error & 9 & $8.951 \mathrm{e}+1$ & $1.796 \mathrm{e}+0$ & $9.252 \mathrm{e}+1$ & Normal \\
\hline NO2-1 & Sampling Error & 9 & $3.169 \mathrm{e}+0$ & $1.056 \mathrm{e}-1$ & $3.335 \mathrm{e}+0$ & Normal \\
\hline NO3-1 & No Sampling Error & 9 & $3.290 \mathrm{e}+0$ & $6.782 \mathrm{e}-2$ & $3.332 \mathrm{e}+0$ & Normal: Student's t \\
\hline SO4-2 & No Sampling Error & 9 & $1.029 \mathrm{e}+0$ & $2.759 \mathrm{e}-2$ & $1.046 \mathrm{e}+0$ & Normal: Student's t \\
\hline
\end{tabular}

Notes: The distribution of NO3 conforms to a normal distribution based on a Wilk-Shapiro goodness of fit test (P-value $=0.3982>\alpha=0.05)$. The UCL95 was based on a Student's $t$ confidence limit as recommended in the ProUCL software guidance. 


\section{APPENDIX C: STATISTICAL RESULTS FOR INORGANIC CONSTITUENTS}

Table 20 Tests and Estimators for Inorganic Constituents

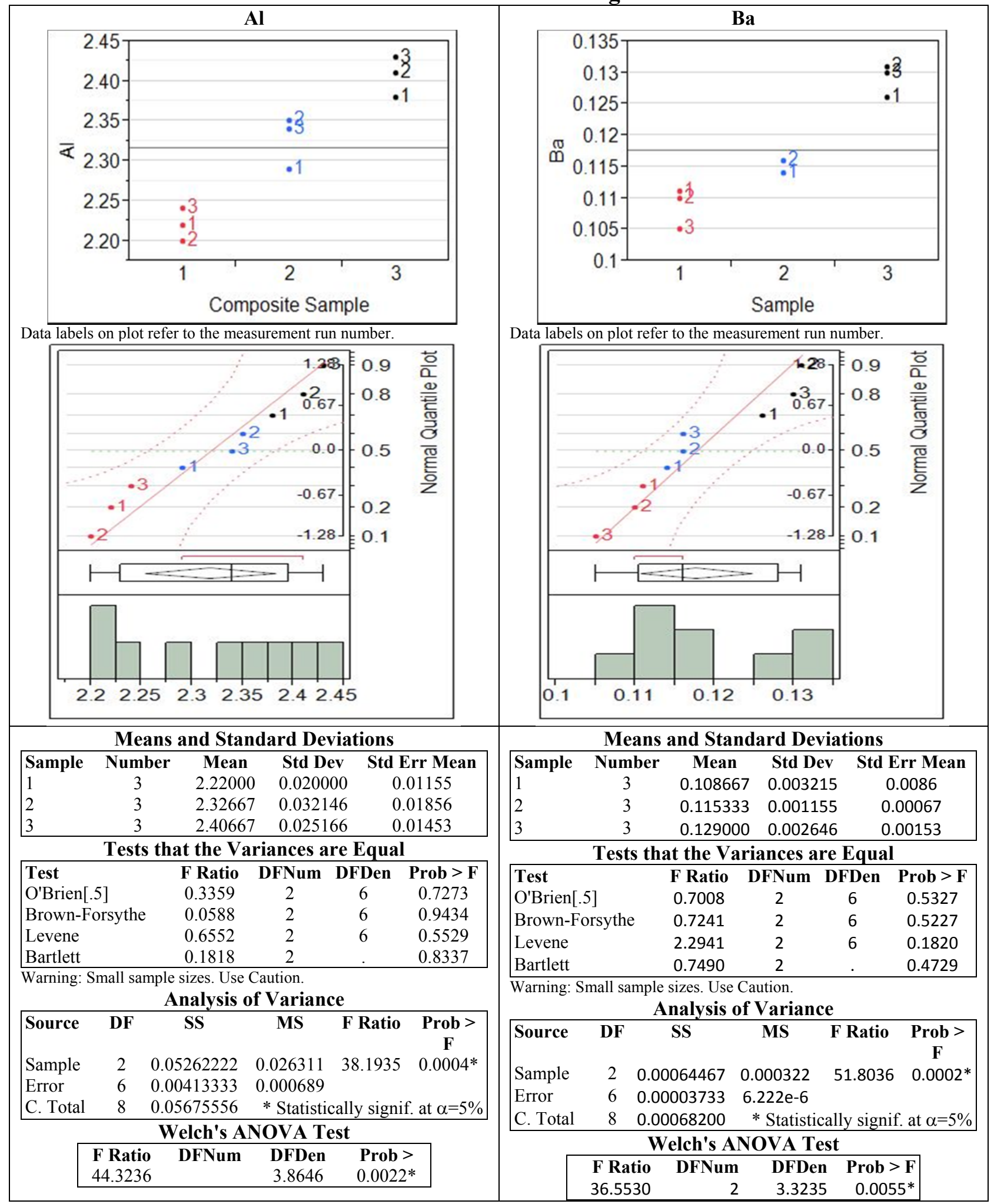




\section{APPENDIX C: STATISTICAL RESULTS FOR INORGANIC CONSTITUENTS}

Table 20 Tests and Estimators for Inorganic Constituents

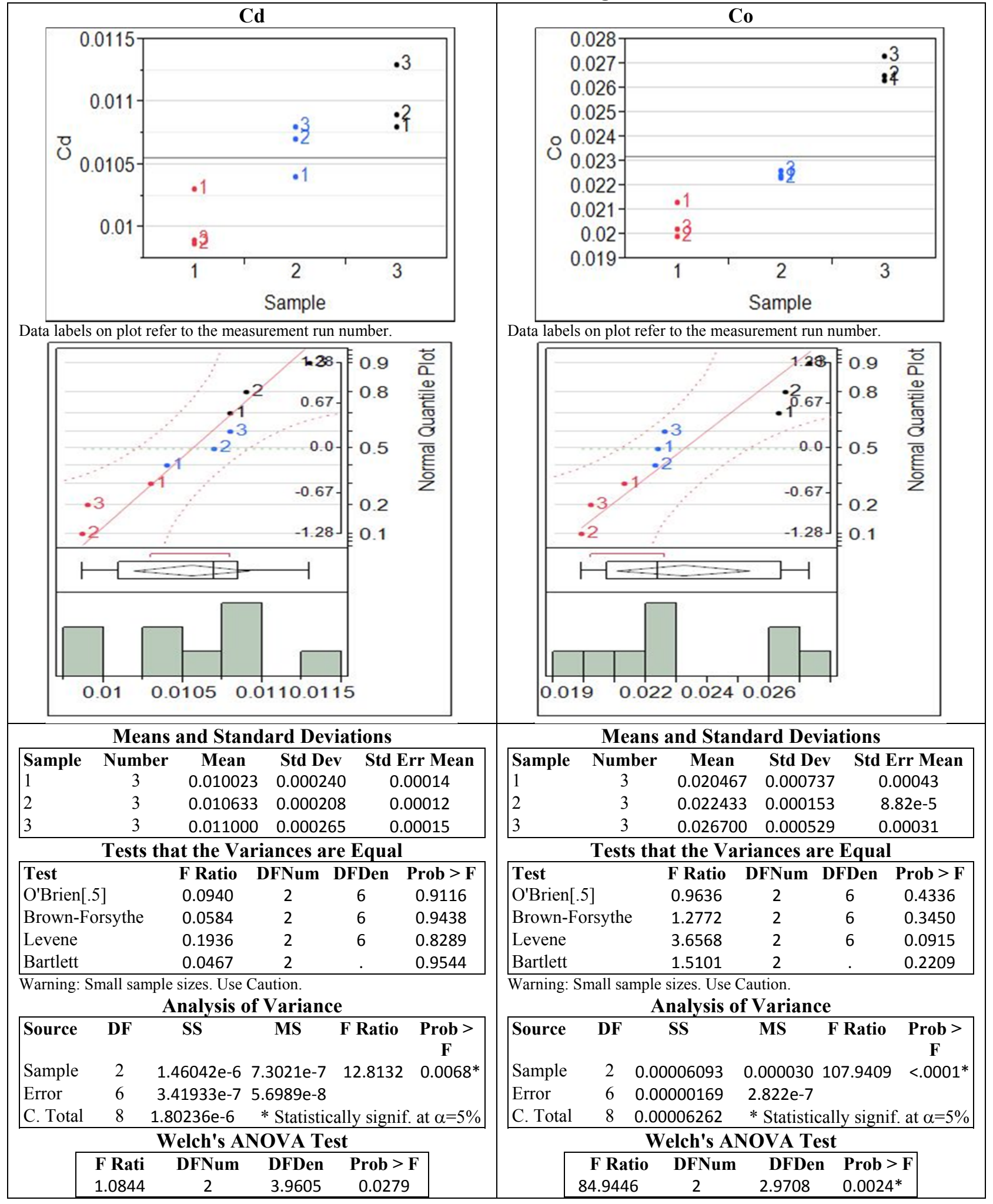


APPENDIX C: STATISTICAL RESULTS FOR INORGANIC CONSTITUENTS Table 20 Tests and Estimators for Inorganic Constituents

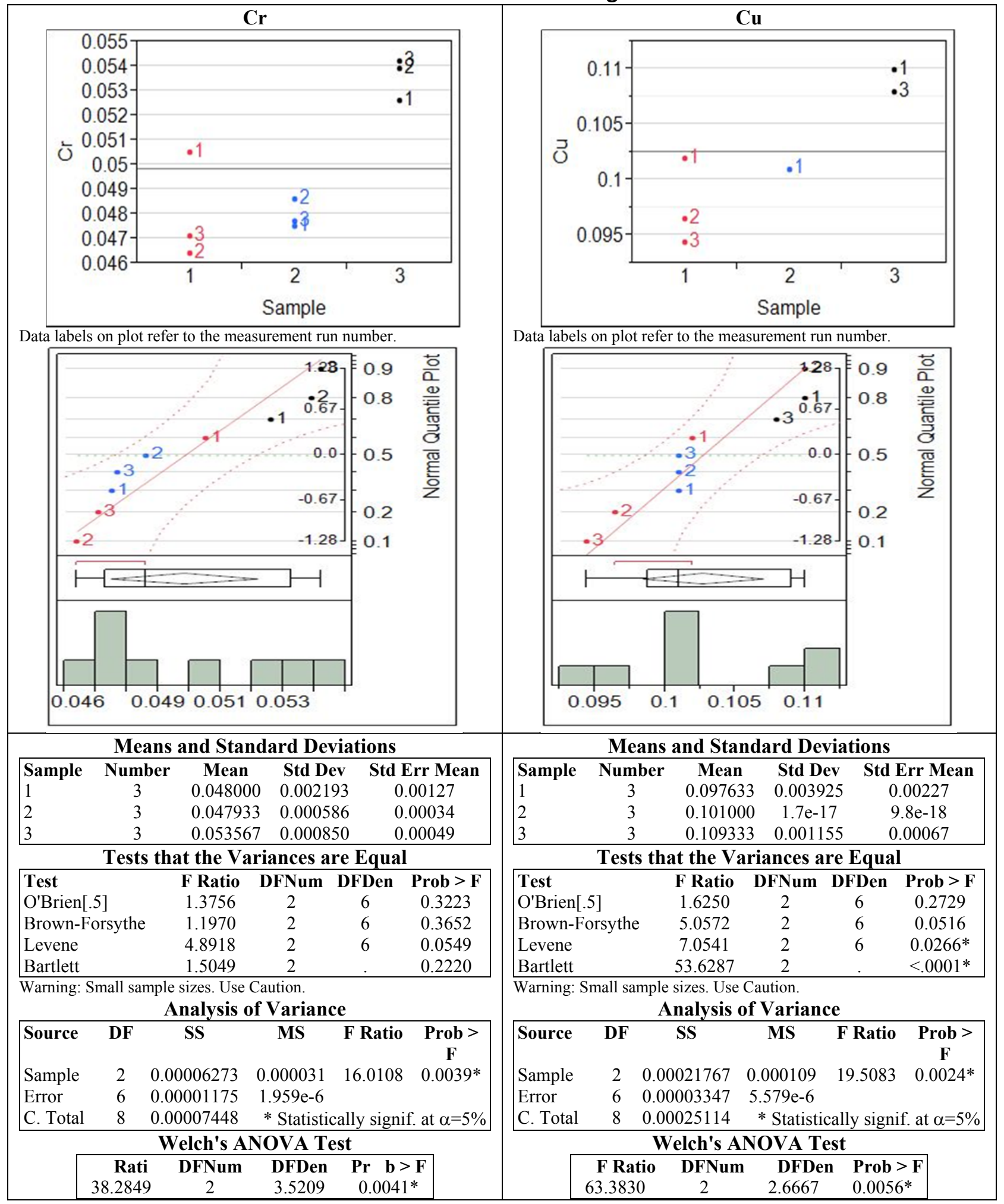


APPENDIX C: STATISTICAL RESULTS FOR INORGANIC CONSTITUENTS

Table 20 Tests and Estimators for Inorganic Constituents

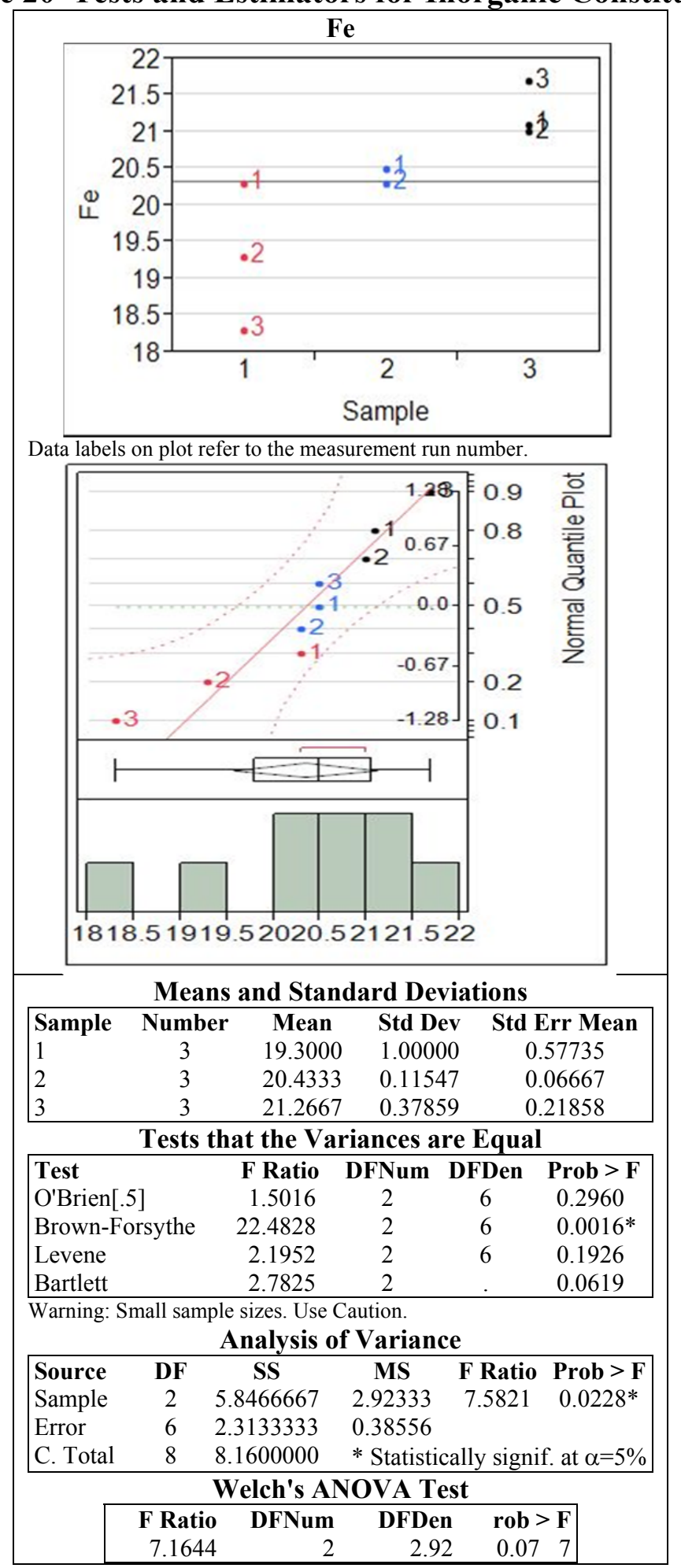




\section{Appendix C: Statistical Results for Inorganic Constituents}

Table 20 Tests and Estimators for Inorganic Constituents

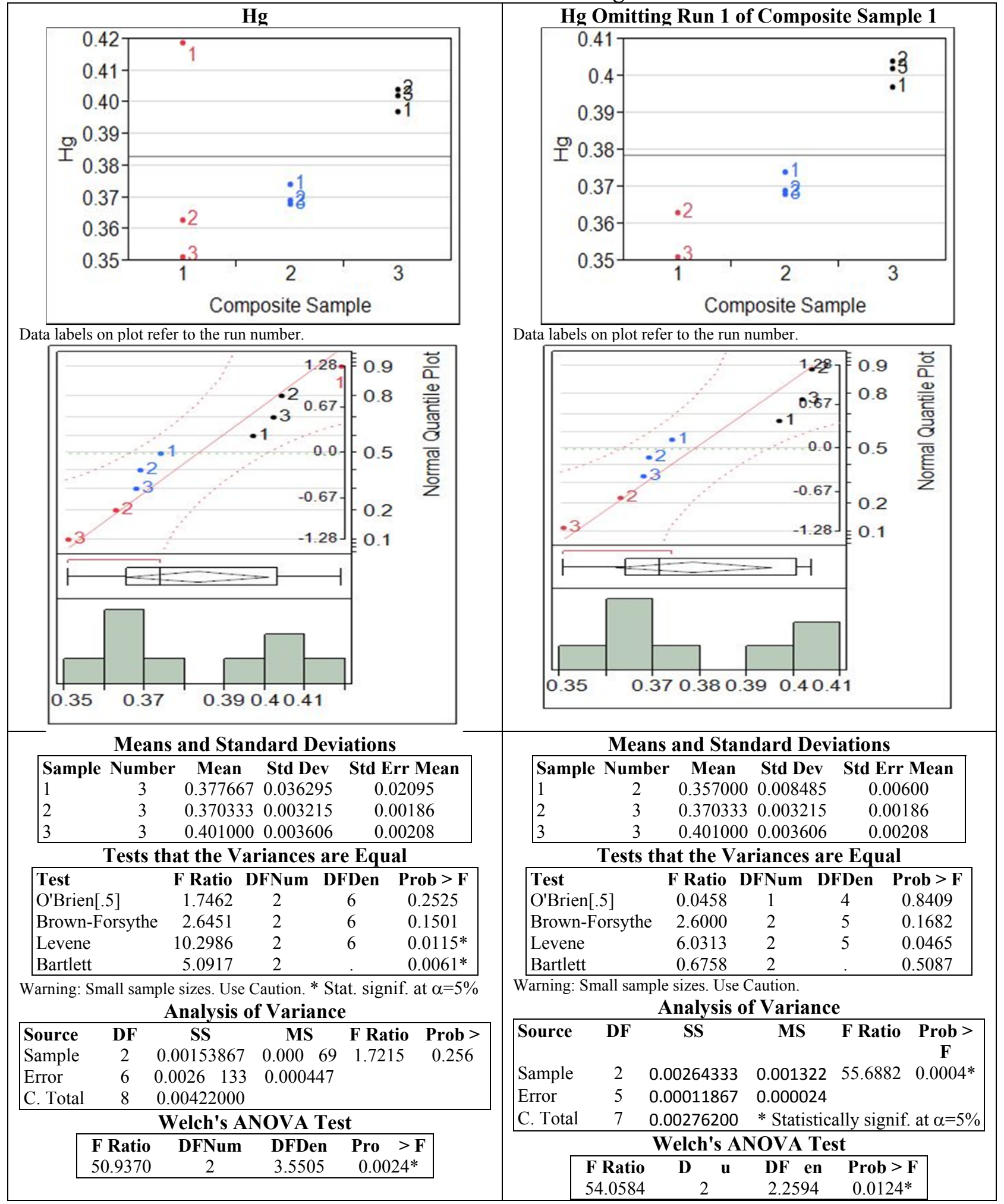




\section{Appendix C: Statistical Results for Inorganic Constituents}

\section{Table 20 Tests and Estimators for Inorganic Constituents}

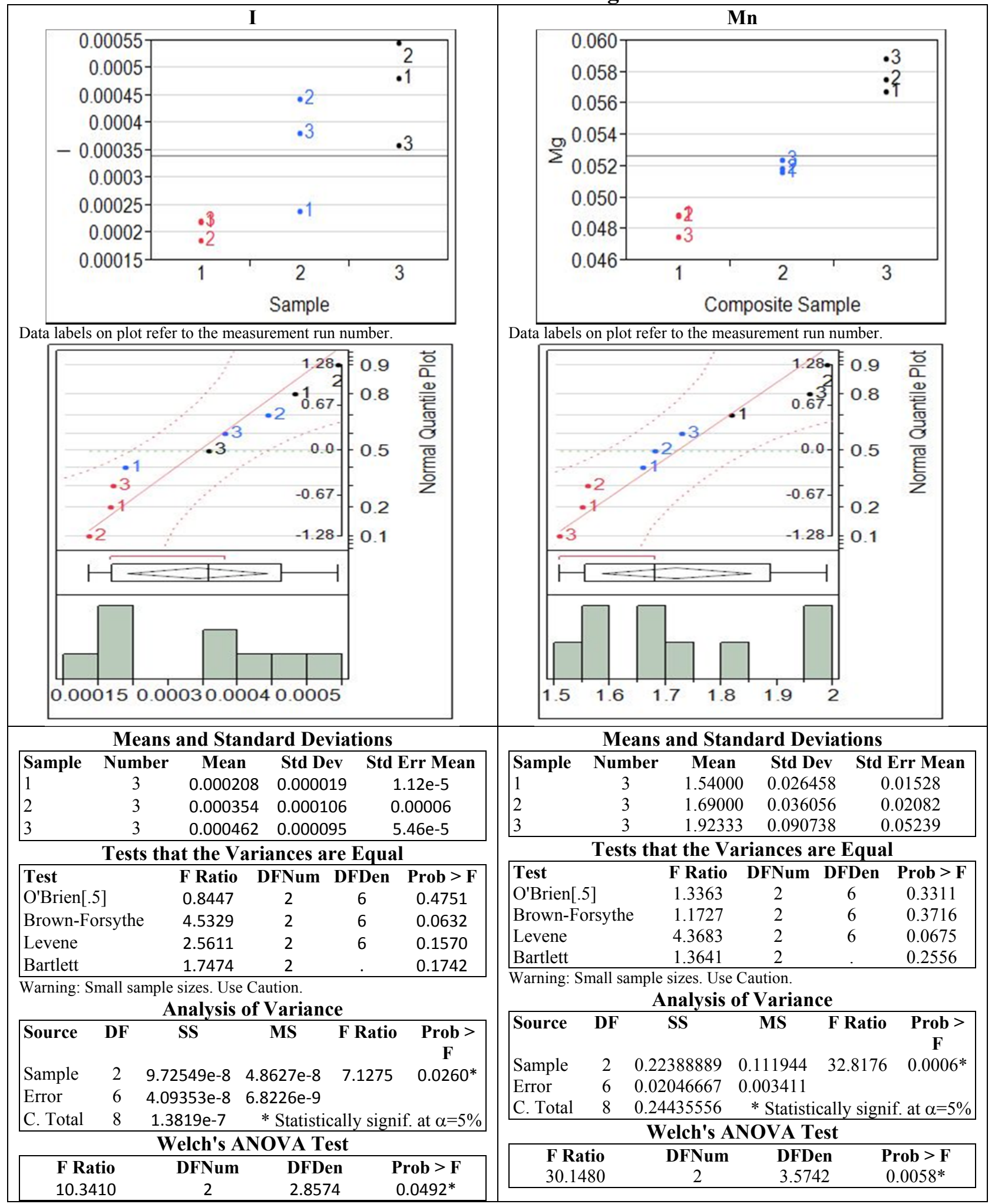




\section{Appendix C: Statistical Results for Inorganic Constituents}

Table 20 Tests and Estimators for Inorganic Constituents

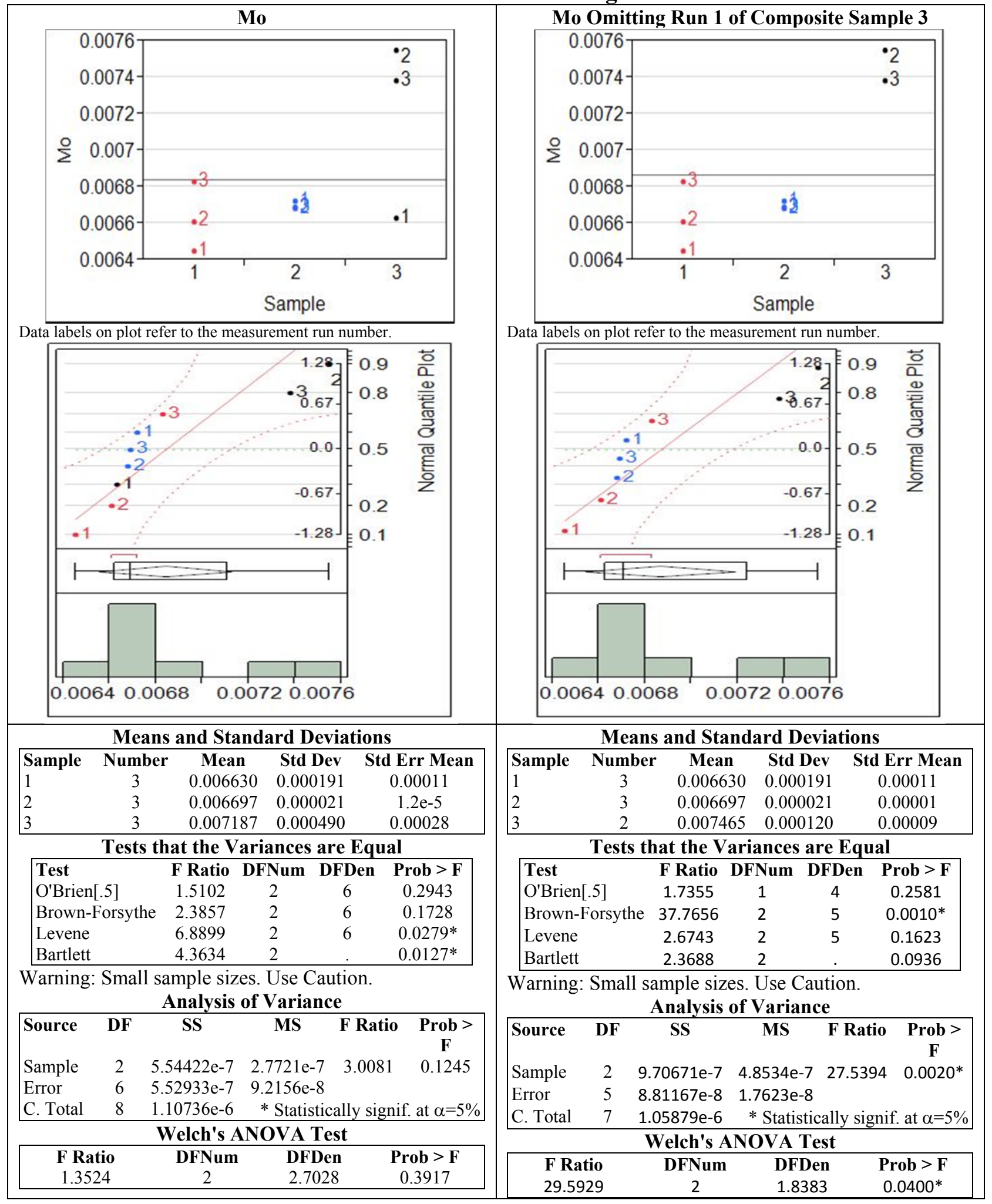




\section{Appendix C: Statistical Results for Inorganic Constituents}

\section{Table 20 Tests and Estimators for Inorganic Constituents}

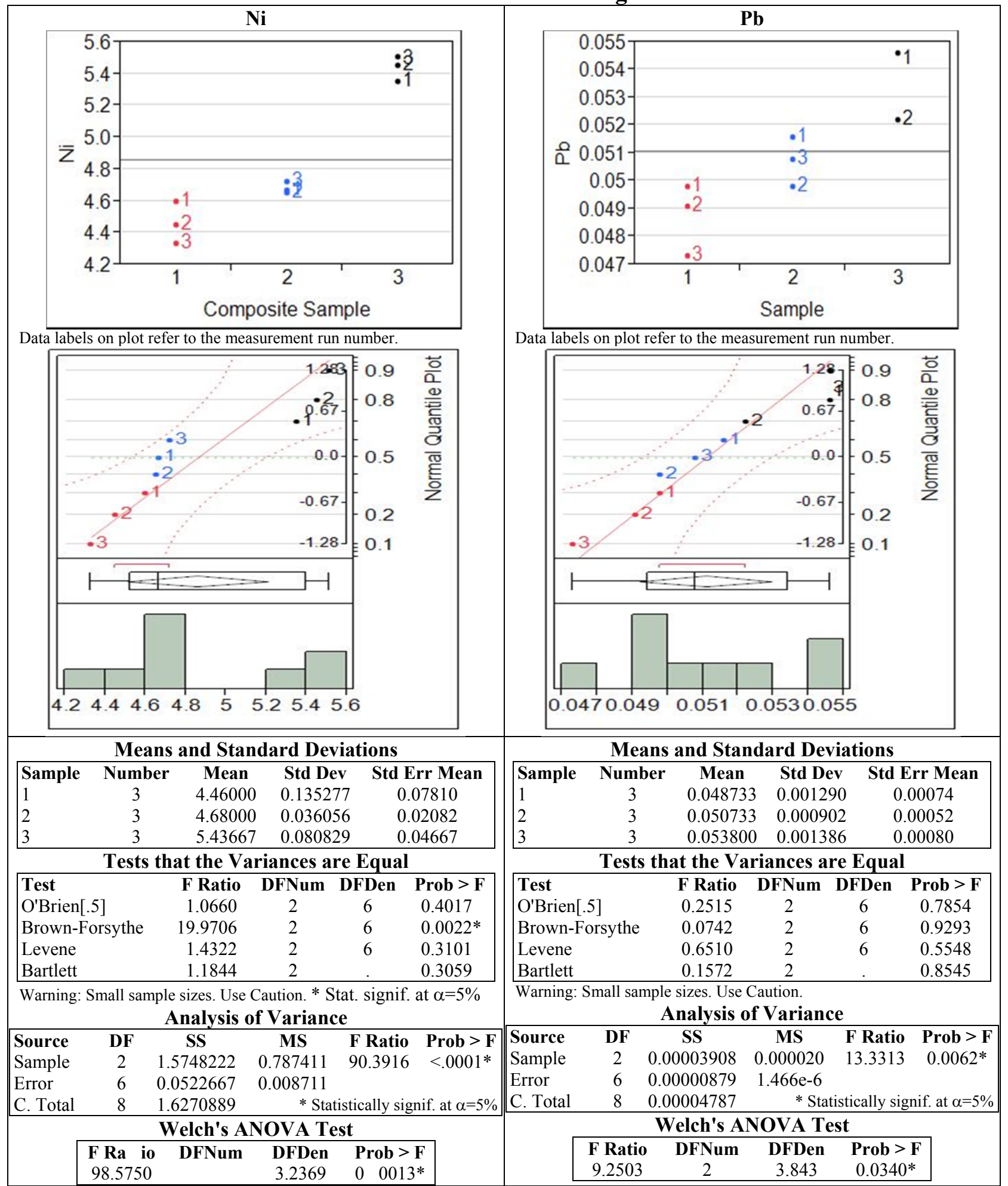




\section{APPENDIX C: STATISTICAL RESULTS FOR INORGANIC CONSTITUENTS} Table 20 Tests and Estimators for Inorganic Constituents

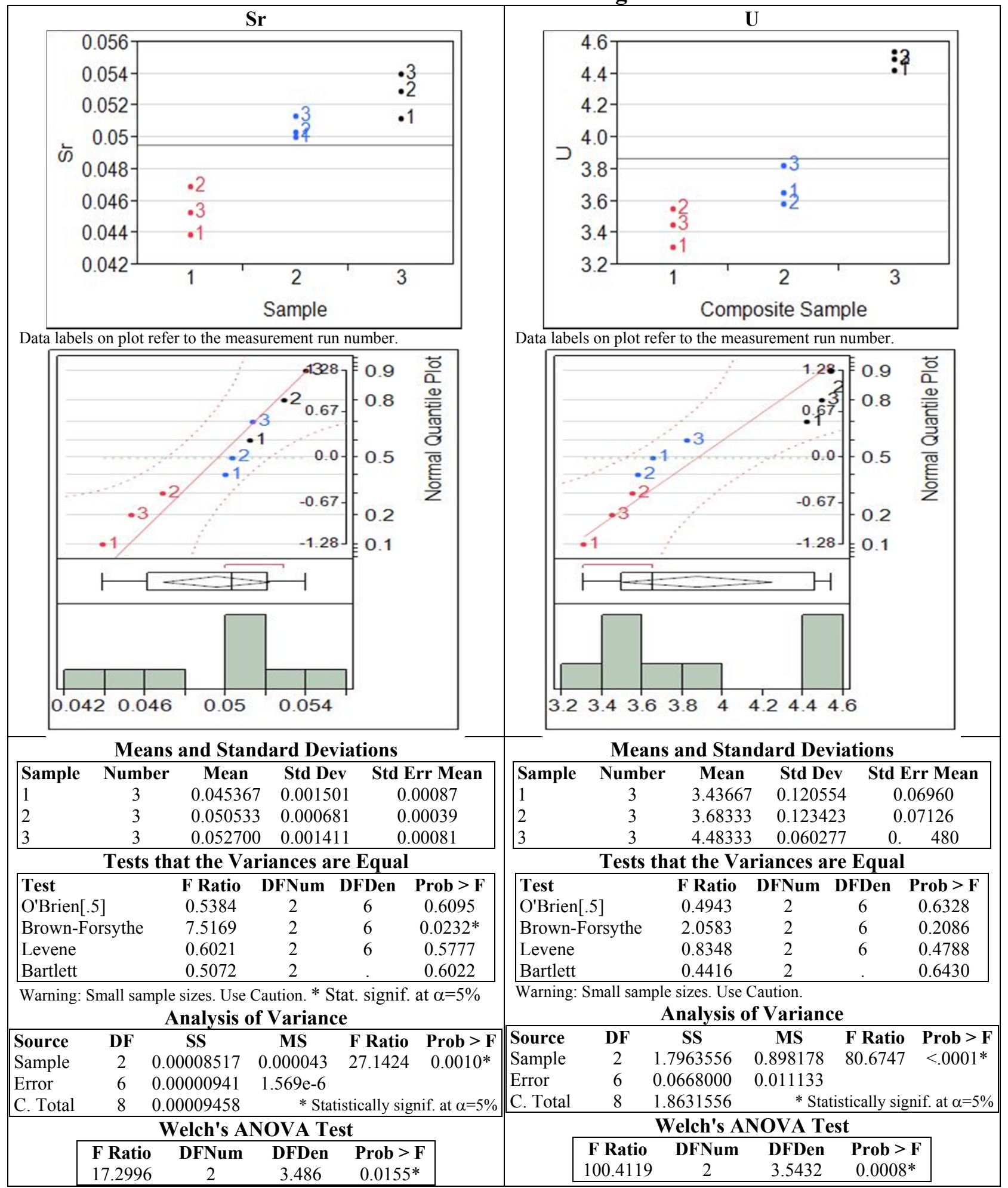


APPENDIX C: STATISTICAL RESULTS FOR INORGANIC CONSTITUENTS Table 20 Tests and Estimators for Inorganic Constituents

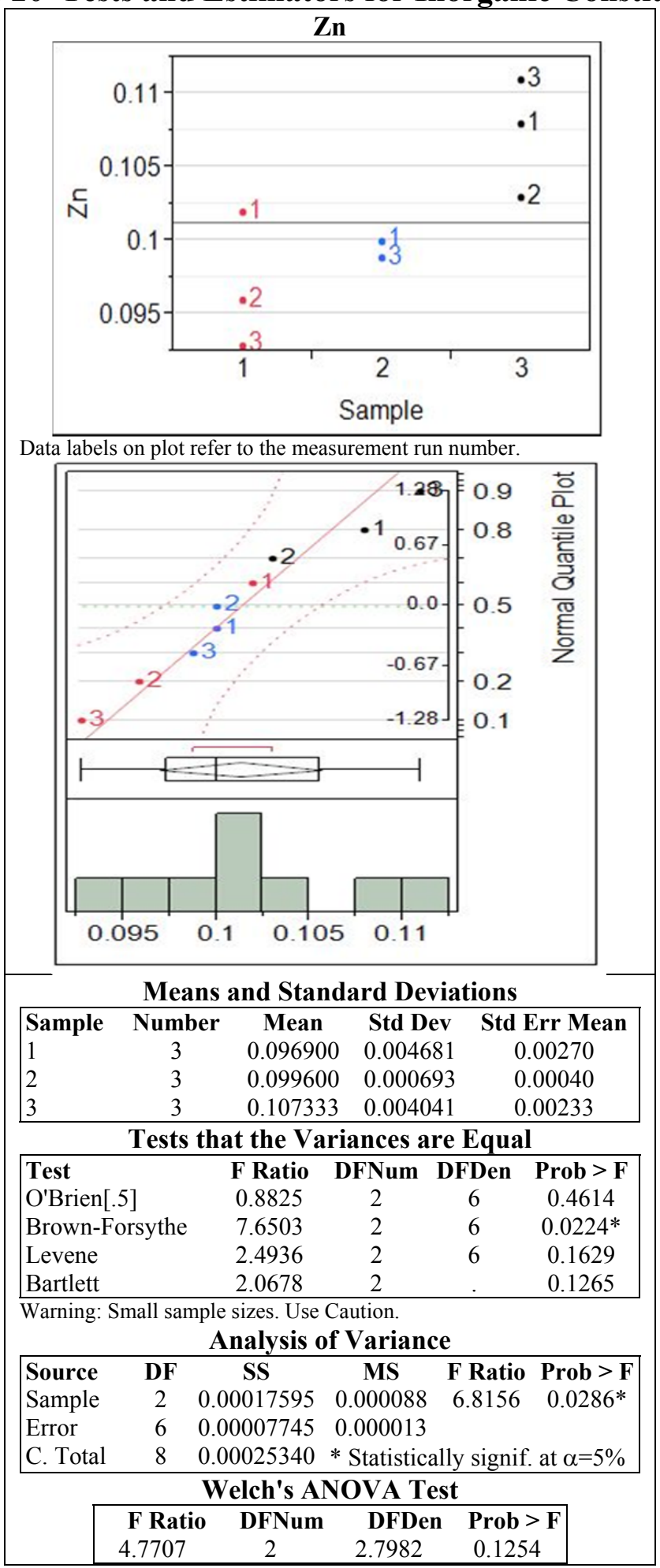




\section{Appendix C: Statistical Results for Inorganic Constituents}

Table 21 Computations for the Mean, Standard Error, and the 95\% Upper Confidence Limit for the Mean Hg Concentration Omitting the Result for Run 1 of Composite Sample 1

ANOVA Summary without Run 1 from Composite 1 of Hg Results

\begin{tabular}{|l|c|l|}
\hline Source & df & Mean Square \\
\hline BS=Between Samples & 2 & $M S_{\text {Sample }}=0.0013216667$ \\
\hline WS=Within Samples & 5 & $M S_{\text {Error }}=0.0000237333$ \\
\hline
\end{tabular}

When the composite samples having differing numbers of measurements, Eqn (4) must be $n_{0}=\left[n-\left(\sum_{i=1}^{m} n_{i}^{2}\right) / n\right] /(m-1)=\left[8-\left(2^{2}+3^{2}+3^{2}\right) / 8\right] /(3-1)=5.25 / 2=2.625$, where

$m=3$ composite samples, $n_{1}=2, n_{2}=3$, and $n_{3}=3$ measurements for composite samples $1,2,3$, respectively, $n=n_{1}+n_{2}+n_{3}=8$ total measurements, and $E\left\{M S_{\text {sample }}\right\}=n_{0} \sigma_{B S}^{2}+\sigma_{w s}^{2}$.

Then $\tilde{V}_{B S}=\left(M S_{\text {Sample }}-M S_{\text {Error }}\right) / n_{0}=(0.001321667-0.0000237333) / 2.625=0.001297934 / 2.625=0.000494451$

and $\tilde{V}_{w S}=M S_{\text {Error }}=0.0000237333$

Estimated Mean, Estimated Std Error of the Mean, and UCL95

$\bar{X}=\sum_{i=1}^{m} \bar{X}_{i} / m=(0.357000+0.370333+0.401000) / 3=1.128333 / 3=0.376111 \mathrm{mg} / \mathrm{g}$

$V\{\bar{X}\}=\left(V\left\{\bar{X}_{1}\right\}+V\left\{\bar{X}_{2}\right\}+V\left\{\bar{X}_{3}\right\}\right) / 3^{2}=\left(\left(V_{B S}+V_{w S} / n_{1}\right)+\left(V_{B S}+V_{w S} / n_{2}\right)+\left(V_{B S}+V_{w S} / n_{3}\right)\right) / 9$

$=\left(3 V_{B S}+(1 / 2+1 / 3+1 / 3) V_{w S}\right) / 9=0.3333 V_{B S}+0.12963 V_{w s}$

$=0.3333(0.000494451)+0.12963(0.000023775)=0.000164815+0.000003082$

$=0.000167897$

Est.std err of the mean $=\sqrt{V\{\bar{X}\}}=\sqrt{0.000167897}=0.01296$

Satterthwaite's $d f=V\{\bar{X}\}^{2} /\left(a_{B S}^{2} V_{B S}^{2} / d f_{B S}+a_{w S}^{2} V_{w S}^{2} / d f_{w S}\right)$

$=0.000167897^{2} /\left((0.33333)^{2}(0.000494451)^{2} / 2+(0.12963)^{2}(0.000023775)^{2} / 5\right)=2.08$

Note: The 95-th percentile of the $t$ distribution with $2.08 \mathrm{df}$ was calculated in JMP $®$ 9.0.0.

$U C L 95=\bar{X}+t_{0.95,2 d f} \sqrt{V\{\bar{X}\}}=0.376111+2.84481924 \sqrt{0.000167897}=0.41297 \mathrm{mg} / \mathrm{g}$ 


\section{Appendix C: Statistical Results for Inorganic Constituents}

Table 22 Computations for the Mean, Standard Error, and the 95\% Upper Confidence Limit for the Mean Mo Concentration Omitting the Result for Run 1 of Composite Sample

3

ANOVA Summary without Run 1 from Composite 1 of Hg Results

\begin{tabular}{|l|c|l|}
\hline Source & df & Mean Square \\
\hline BS=Between Samples & 2 & $M S_{\text {Sample }}=4.8533542 \mathrm{e}-7$ \\
\hline WS=Within Samples & 5 & $M S_{\text {Error }}=1.7623333 \mathrm{e}-8$ \\
\hline
\end{tabular}

When the composite samples having differing numbers of measurements, Eqn (4) must be

$n_{0}=\left[n-\left(\sum_{i=1}^{m} n_{i}^{2}\right) / n\right] /(m-1)=\left[8-\left(3^{2}+3^{2}+2^{2}\right) / 8\right] /(3-1)=5.25 / 2=2.625$, where

$m=3$ composite samples, $n_{1}=3, n_{2}=3$, and $n_{3}=2$ measurements for composite samples $1,2,3$, respectively,

$n=n_{1}+n_{2}+n_{3}=8$ total measurements, and $E\left\{M S_{\text {sample }}\right\}=n_{0} \sigma_{B S}^{2}+\sigma_{w s}^{2}$.

Then $\tilde{V}_{B S}=\left(M S_{\text {sample }}-M S_{\text {Error }}\right) / n_{0}=(4.8533542 \mathrm{e}-7-1.7623333 \mathrm{e}-8) / 2.625=4.677121 e-7 / 2.625=1.781760 e-7$

and $\tilde{V}_{W S}=M S_{\text {Error }}=1.7623333 \mathrm{e}-8$

Estimated Mean, Estimated Std Error of the Mean, and UCL95

$\bar{X}=\sum_{i=1}^{m} \bar{X}_{i} / m=(0.006630+0.006697+0.007465) / 3=0.020792 / 3=0.006931 \mathrm{mg} / \mathrm{g}$

$V\{\bar{X}\}=\left(V\left\{\bar{X}_{1}\right\}+V\left\{\bar{X}_{2}\right\}+V\left\{\bar{X}_{3}\right\}\right) / 3^{2}=\left(\left(V_{B S}+V_{w S} / n_{1}\right)+\left(V_{B S}+V_{w S} / n_{2}\right)+\left(V_{B S}+V_{w S} / n_{3}\right)\right) / 9$

$=\left(3 V_{B S}+(1 / 3+1 / 3+1 / 2) V_{w S}\right) / 9=0.3333 V_{B S}+0.12963 V_{w s}$

$=0.3333(1.781760 e-7)+0.12963(1.7623333 e-8)=5.938607 e-8+2.284513 e-9$

$=6.167058 e-8$

Est.st d err of the mean $=\sqrt{V\{\bar{X}\}}=\sqrt{6.167058 e-8}=0.000248336$

Satterthwaite's $d f=V\{\bar{X}\}^{2} /\left(a_{B S}^{2} V_{B S}^{2} / d f_{B S}+a_{w s}^{2} v_{w S}^{2} / d f_{w S}\right)$

$=(6.167058 e-8)^{2} /\left((0.33333)^{2}(1.781760 e-7)^{2} / 2+(0.12963)^{2}(1.7623333 e-8)^{2} / 5\right)=2.16$

Note: The 95-th percentile of the $t$ distribution with $2.08 \mathrm{df}$ was calculated in JMP $®$ 9.0.0.

$U C L 95=\bar{X}+t_{0.95,2 \text { df }} \sqrt{V\{\bar{X}\}}=0.006931+2.777697 \sqrt{6.167058 e-8}=0.0076208 \mathrm{mg} / \mathrm{g}$ 


\section{Appendix C: Statistical Results for Inorganic Constituents}

Table 23 Inorganic Constituent Concentrations with All Results Less Than Their MDCs

\begin{tabular}{|l|c|c|}
\hline & \multicolumn{2}{|c|}{ MDC } \\
\hline Inorganic & Min & Max \\
\hline $\mathrm{Ag}$ & $1.12 \mathrm{E}-02$ & $1.15 \mathrm{E}-02$ \\
\hline $\mathrm{As}$ & $5.33 \mathrm{E}-04$ & $5.49 \mathrm{E}-04$ \\
\hline $\mathrm{B}$ & $1.57 \mathrm{E}-02$ & $1.61 \mathrm{E}-02$ \\
\hline $\mathrm{Sb}$ & $9.90 \mathrm{E}-02$ & $1.02 \mathrm{E}-01$ \\
\hline $\mathrm{Se}$ & $1.07 \mathrm{E}-03$ & $1.10 \mathrm{E}-03$ \\
\hline
\end{tabular}




\section{Appendix C: Statistical Results for Inorganic Constituents}

Table 24 95\% UCLs for Mean Concentrations of Inorganic Constituents with All Results Above Their MDCs

\begin{tabular}{|c|l|c|c|c|c|}
\hline Inorganic & Model Type & $\mathbf{N}$ & Mean & Standard Deviation & UCL95 \\
\hline $\mathrm{Al}$ & Sampling Error & 9 & $2.318 \mathrm{e}+0$ & $9.607 \mathrm{e}-2$ & $2.476 \mathrm{e}+0$ \\
\hline $\mathrm{Ba}$ & Sampling Error & 9 & $1.177 \mathrm{e}-1$ & $1.056 \mathrm{e}-2$ & $1.351 \mathrm{e}-1$ \\
\hline $\mathrm{Cd}$ & Sampling Error & 9 & $1.055 \mathrm{e}-2$ & $5.305 \mathrm{e}-4$ & $1.138 \mathrm{e}-2$ \\
\hline $\mathrm{Co}$ & Sampling Error & 9 & $2.320 \mathrm{e}-2$ & $3.216 \mathrm{e}-3$ & $2.857 \mathrm{e}-2$ \\
\hline $\mathrm{Cr}$ & Sampling Error & 9 & $4.983 \mathrm{e}-2$ & $3.429 \mathrm{e}-3$ & $5.528 \mathrm{e}-2$ \\
\hline $\mathrm{Cu}$ & Sampling Error & 9 & $1.027 \mathrm{e}-1$ & $6.324 \mathrm{e}-3$ & $1.128 \mathrm{e}-1$ \\
\hline $\mathrm{Fe}$ & Sampling Error & 9 & $2.033 \mathrm{e}+1$ & $1.110 \mathrm{e}+0$ & $2.200 \mathrm{e}+1$ \\
\hline $\mathrm{Hg}$ & No Sampling Error & 9 & $3.830 \mathrm{e}-1$ & $2.297 \mathrm{e}-2$ & $3.972 \mathrm{e}-1$ \\
\hline $\mathrm{Hg}$ & Sampling Error & 8 & $3.761 \mathrm{e}-1$ & $2.276 \mathrm{e}-2$ & $4.130 \mathrm{e}-1$ \\
\hline $\mathrm{I}$ & Sampling Error & 9 & $3.416 \mathrm{e}-4$ & $1.441 \mathrm{e}-4$ & $5.562 \mathrm{e}-4$ \\
\hline $\mathrm{Mn}$ & Sampling Error & 9 & $1.718 \mathrm{e}+0$ & $1.990 \mathrm{e}-1$ & $2.043 \mathrm{e}+0$ \\
\hline $\mathrm{Mo}$ & No Sampling Error & 9 & $6.838 \mathrm{e}-3$ & $3.720 \mathrm{e}-4$ & $7.068 \mathrm{e}-3$ \\
\hline $\mathrm{Mo}$ & Sampling Error & 8 & $6.931 \mathrm{e}-3$ & $4.425 \mathrm{e}-4$ & $7.621 \mathrm{e}-3$ \\
\hline $\mathrm{Ni}$ & Sampling Error & 9 & $4.859 \mathrm{e}+0$ & $5.180 \mathrm{e}-1$ & $5.723 \mathrm{e}+0$ \\
\hline $\mathrm{Pb}$ & Sampling Error & 9 & $5.109 \mathrm{e}-2$ & $2.737 \mathrm{e}-3$ & $5.539 \mathrm{e}-2$ \\
\hline $\mathrm{Sr}$ & Sampling Error & 9 & $4.953 \mathrm{e}-2$ & $3.904 \mathrm{e}-3$ & $5.588 \mathrm{e}-2$ \\
\hline $\mathrm{U}$ & Sampling Error & 9 & $3.868 \mathrm{e}+0$ & $5.539 \mathrm{e}-1$ & $4.790 \mathrm{e}+0$ \\
\hline $\mathrm{Zn}$ & Sampling Error & 9 & $1.013 \mathrm{e}-1$ & $6.159 \mathrm{e}-3$ & $1.104 \mathrm{e}-1$ \\
\hline
\end{tabular}

• Hg calculations in this row omitted a potential outlier associated with Run 1 from Composite Sample 1.

- Mo calculations in this row omitted a potential outlier associated with Run 1 from Composite Sample 3. 


\section{Appendix C: Statistical Results for Radionuclide Constituents}

\section{Table 25 Tests and Estimators for Radionuclides}

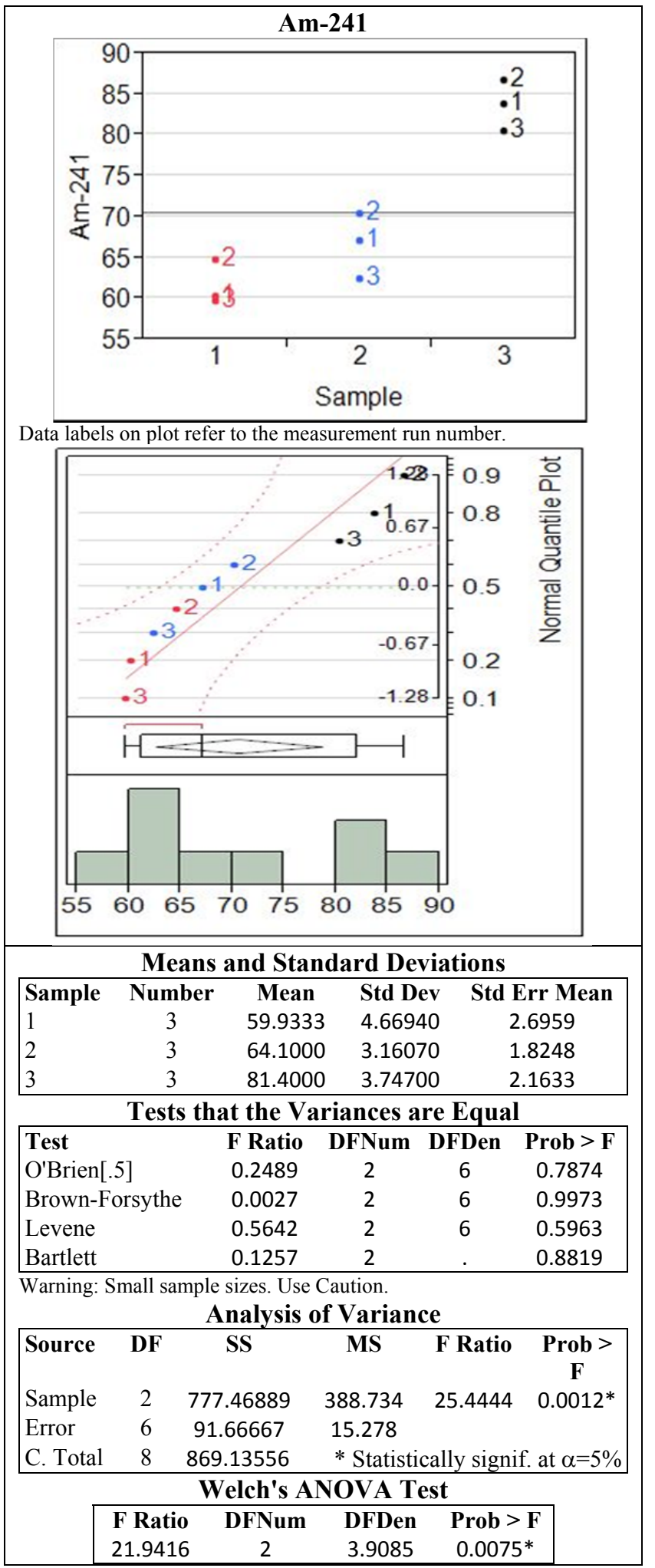




\section{Appendix C: Statistical Results for Radionuclide Constituents}

Table 25 Tests and Estimators for Radionuclides

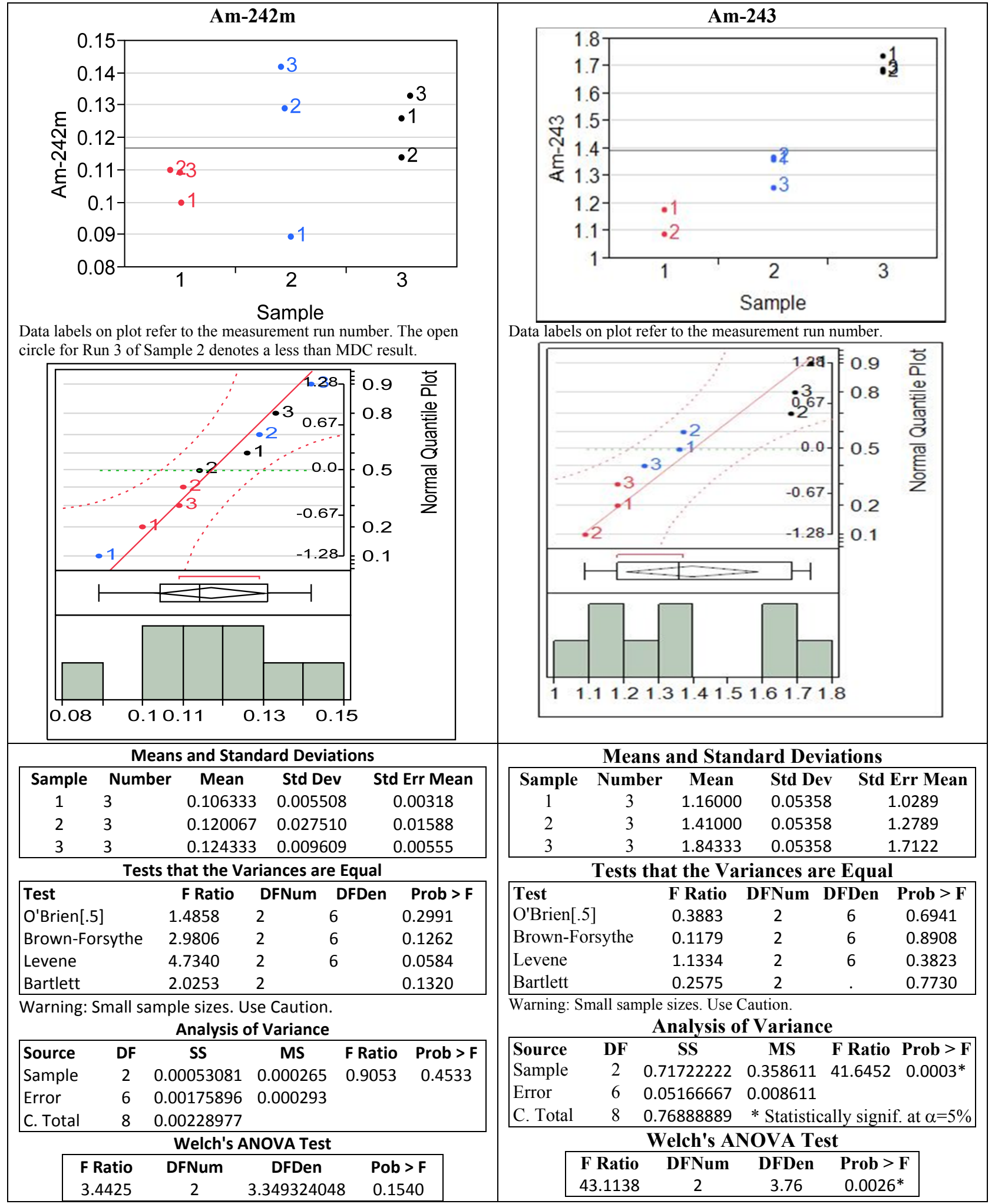




\section{Appendix C: Statistical Results for Radionuclide Constituents}

Table 25 Tests and Estimators for Radionuclides

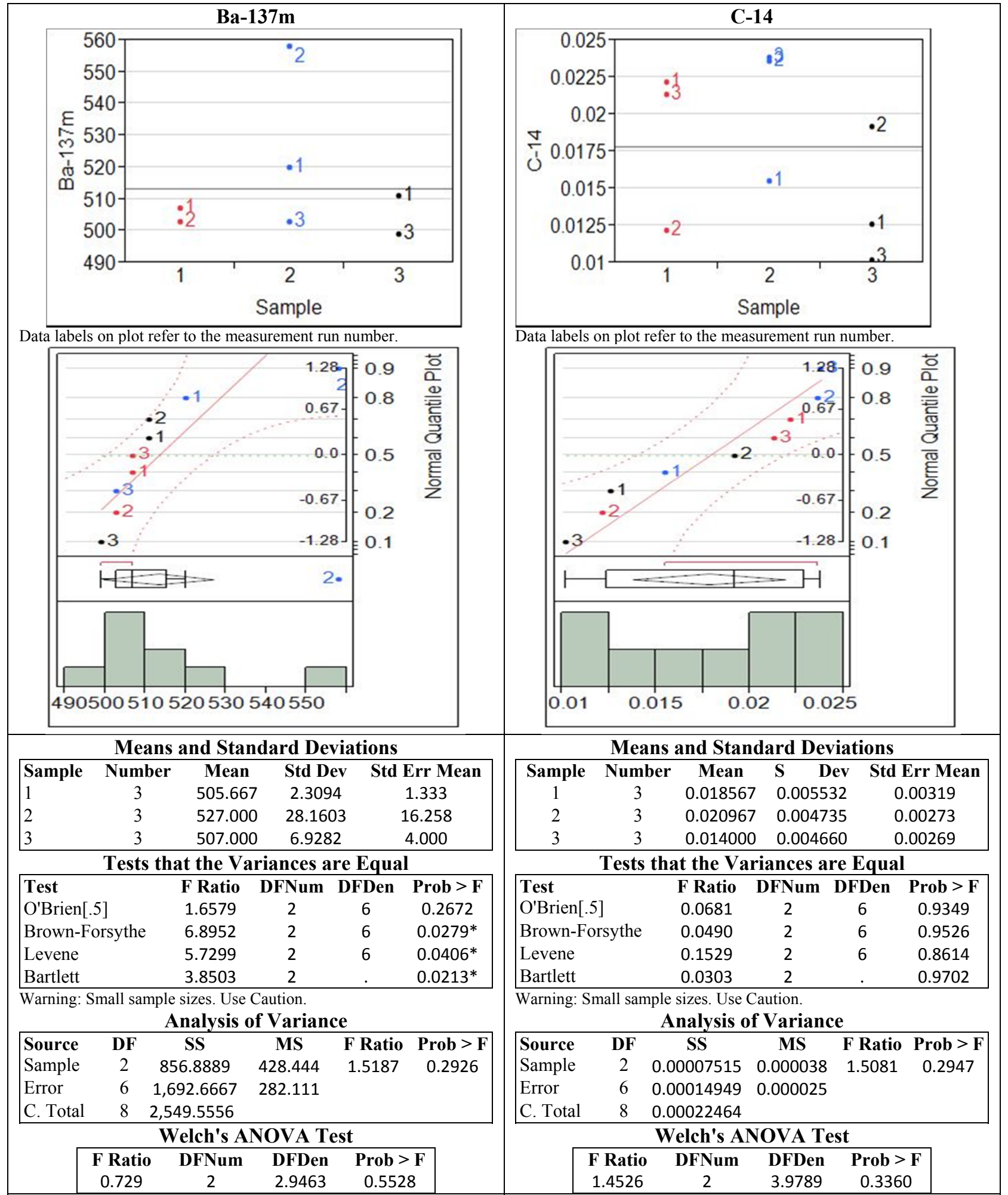




\section{Appendix C: Statistical Results for Radionuclide Constituents}

Table 25 Tests and Estimators for Radionuclides

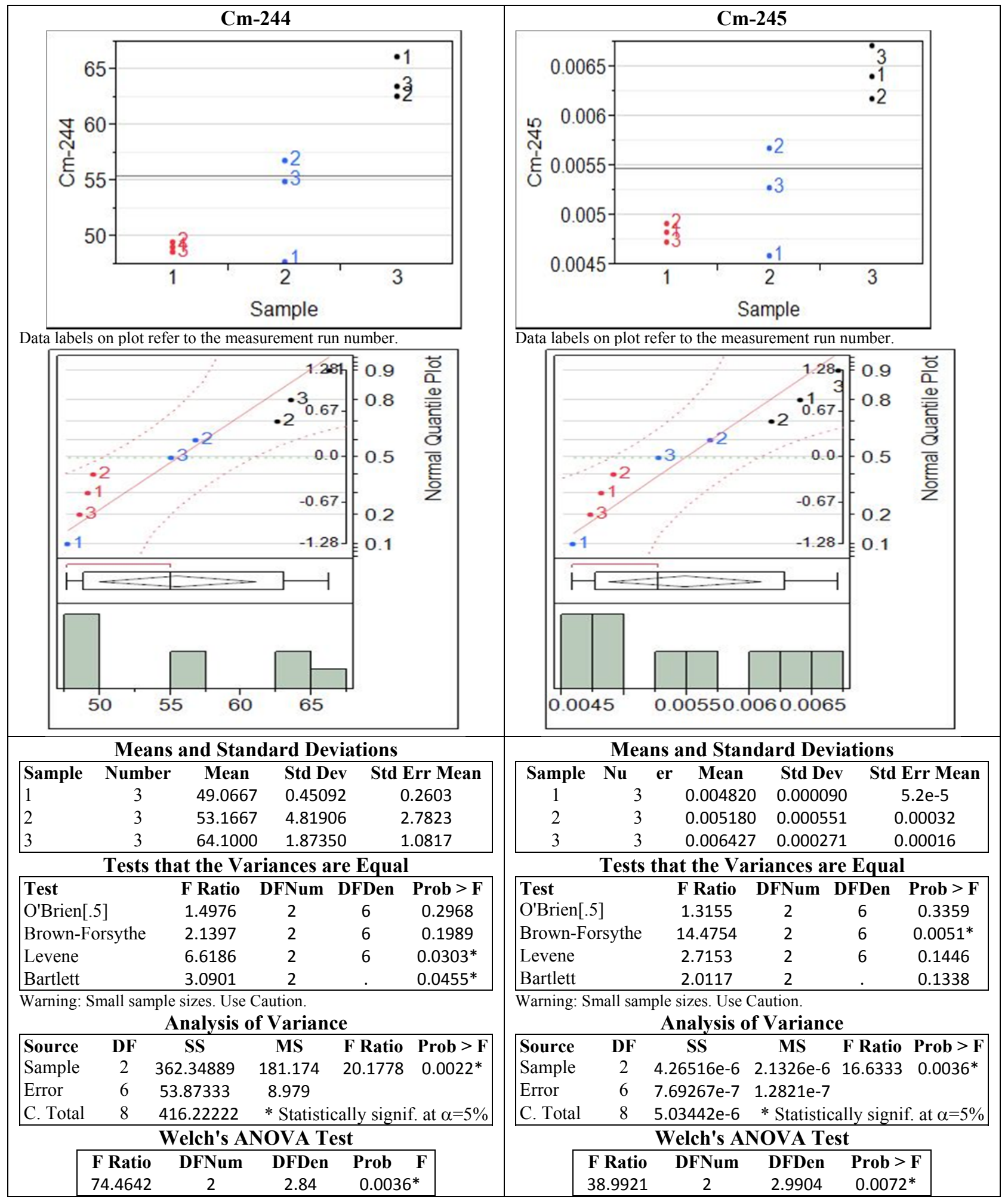




\section{Appendix C: Statistical Results for Radionuclide Constituents}

Table 25 Tests and Estimators for Radionuclides

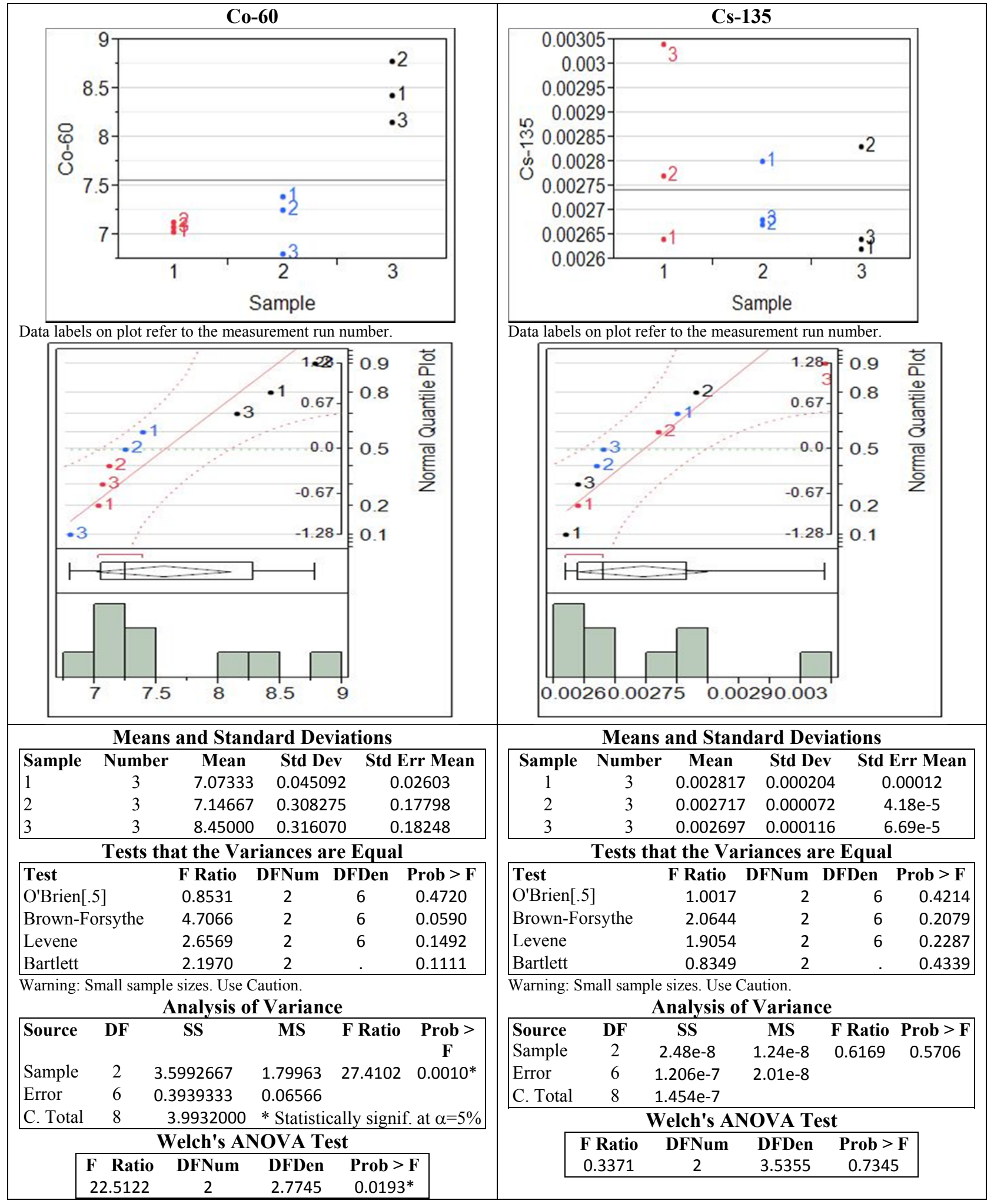




\section{Appendix C: Statistical Results for Radionuclide Constituents}

Table 25 Tests and Estimators for Radionuclides

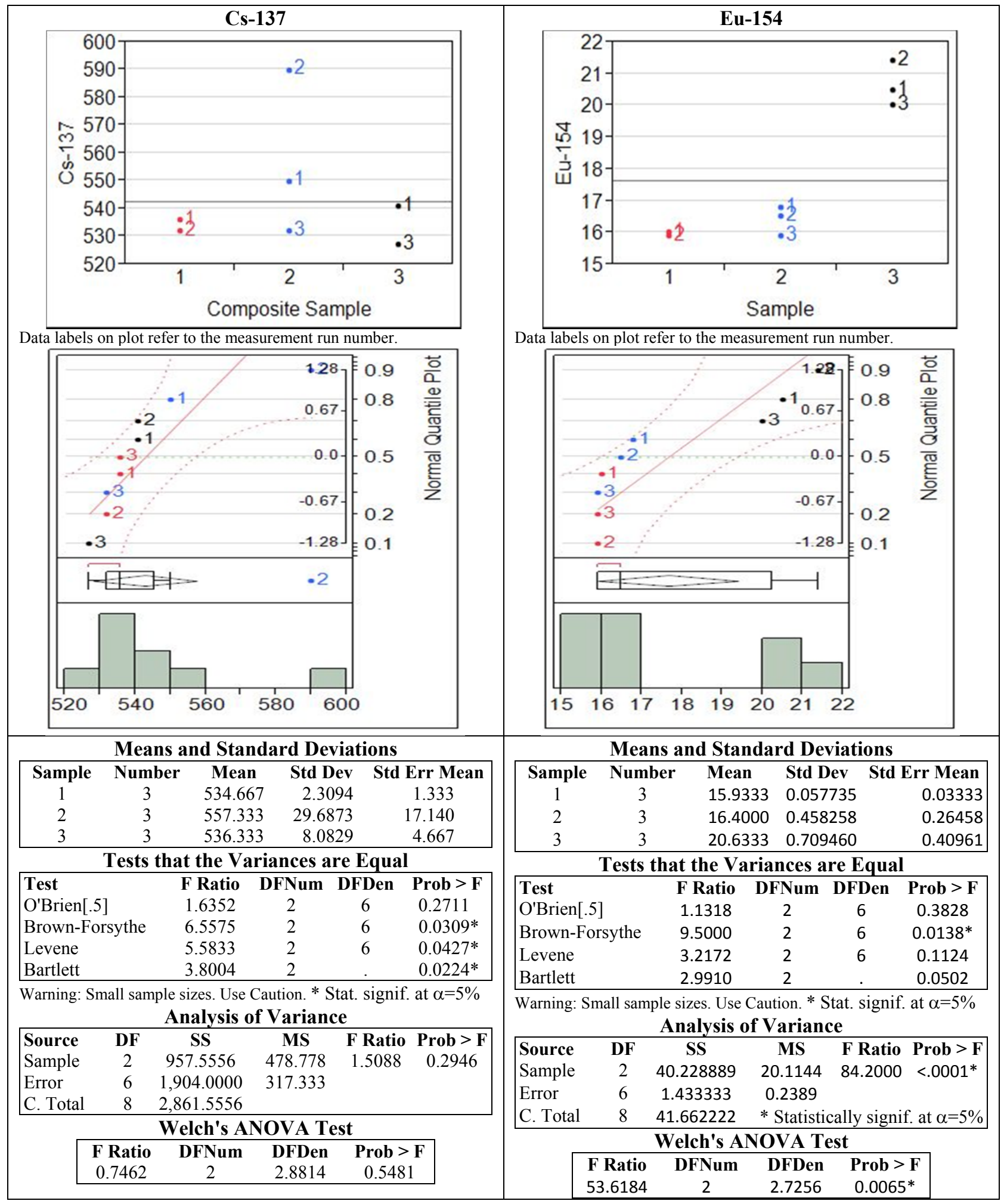




\section{Appendix C: Statistical Results for Radionuclide Constituents}

Table 25 Tests and Estimators for Radionuclides

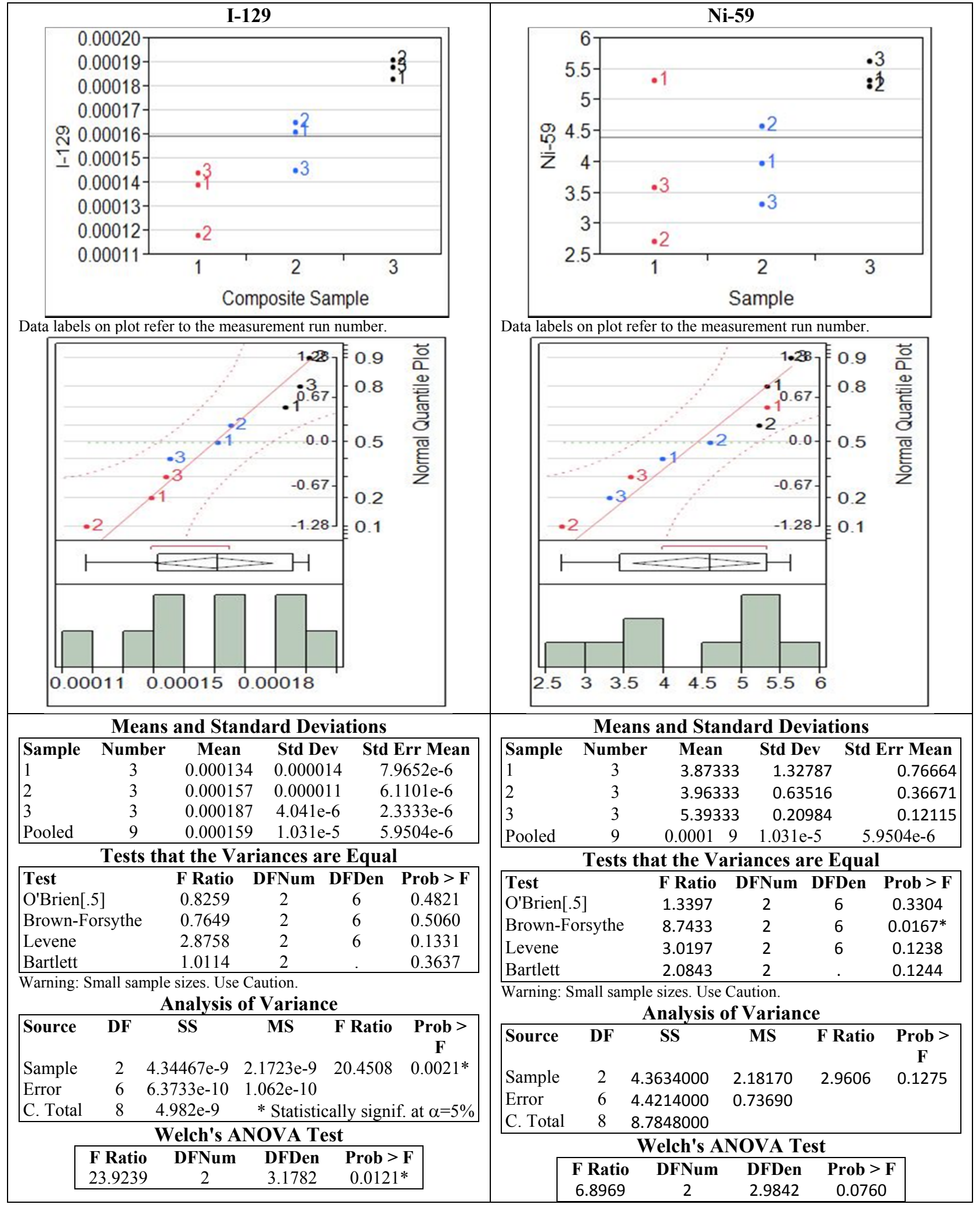




\section{Appendix C: Statistical Results for Radionuclide Constituents}

Table 25 Tests and Estimators for Radionuclides

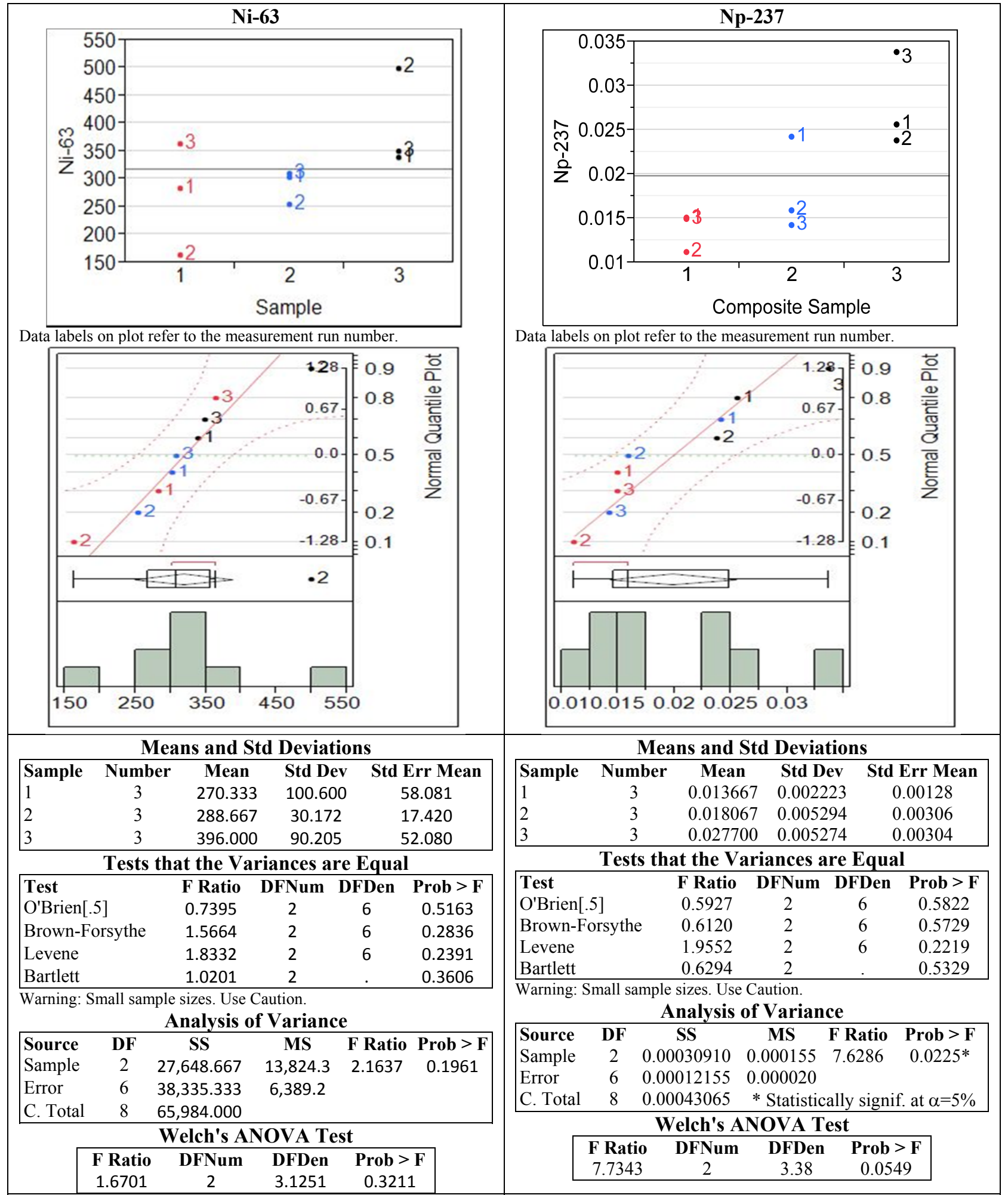




\section{Appendix C: Statistical Results for Radionuclide Constituents}

Table 25 Tests and Estimators for Radionuclides

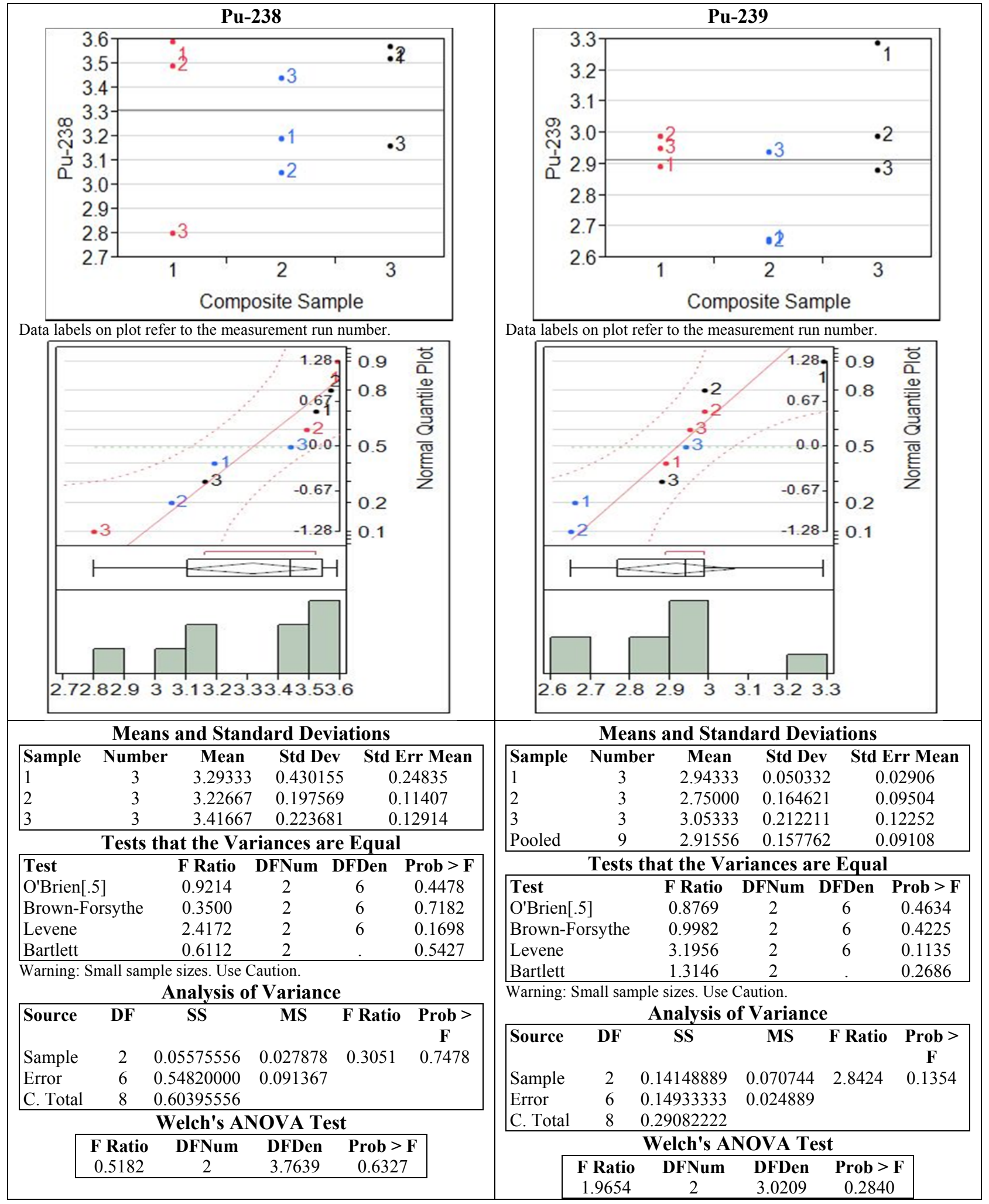




\section{Appendix C: Statistical Results for Radionuclide Constituents}

Table 25 Tests and Estimators for Radionuclides

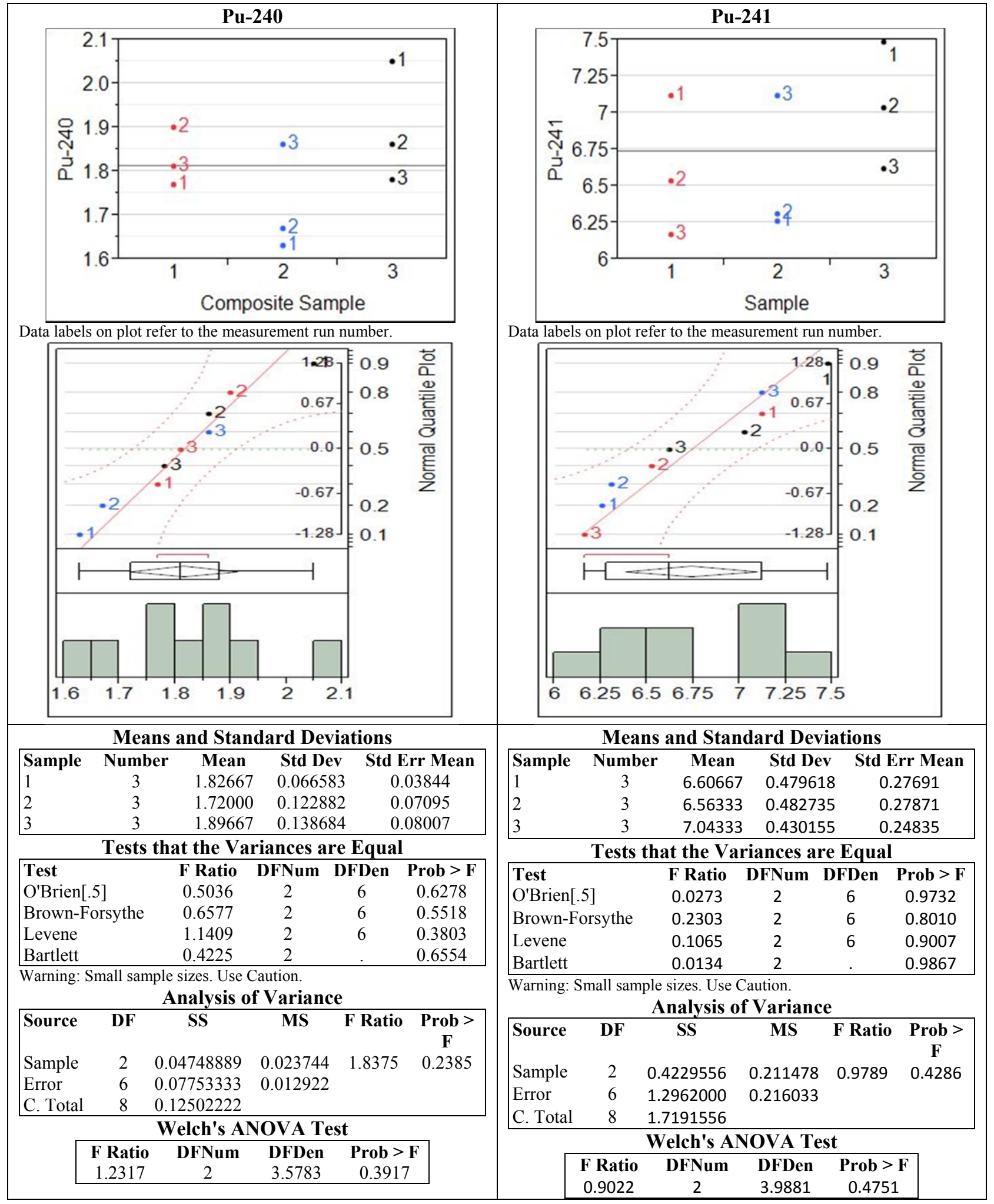




\section{Appendix C: Statistical Results for Radionuclide Constituents}

\section{Table 25 Tests and Estimators for Radionuclides}

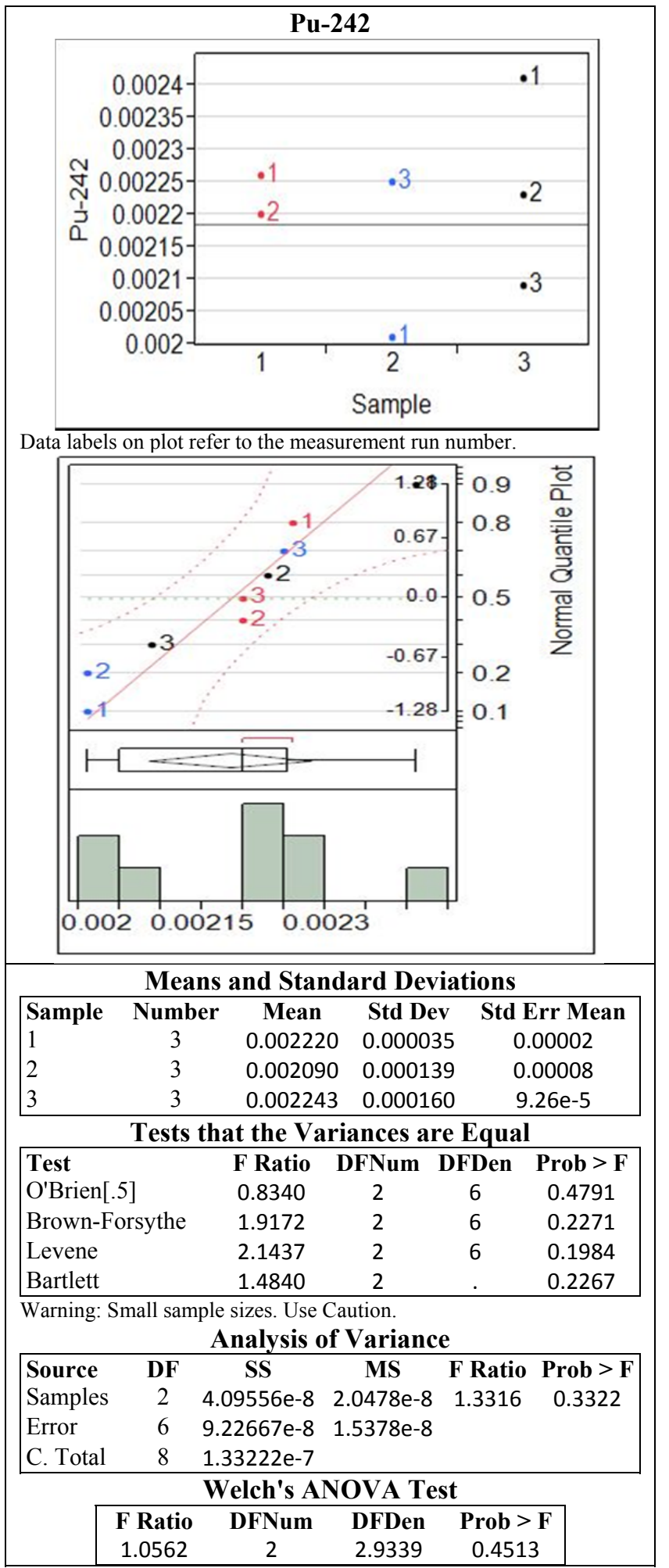




\section{Appendix C: Statistical Results for Radionuclide Constituents}

Table 25 Tests and Estimators for Radionuclides

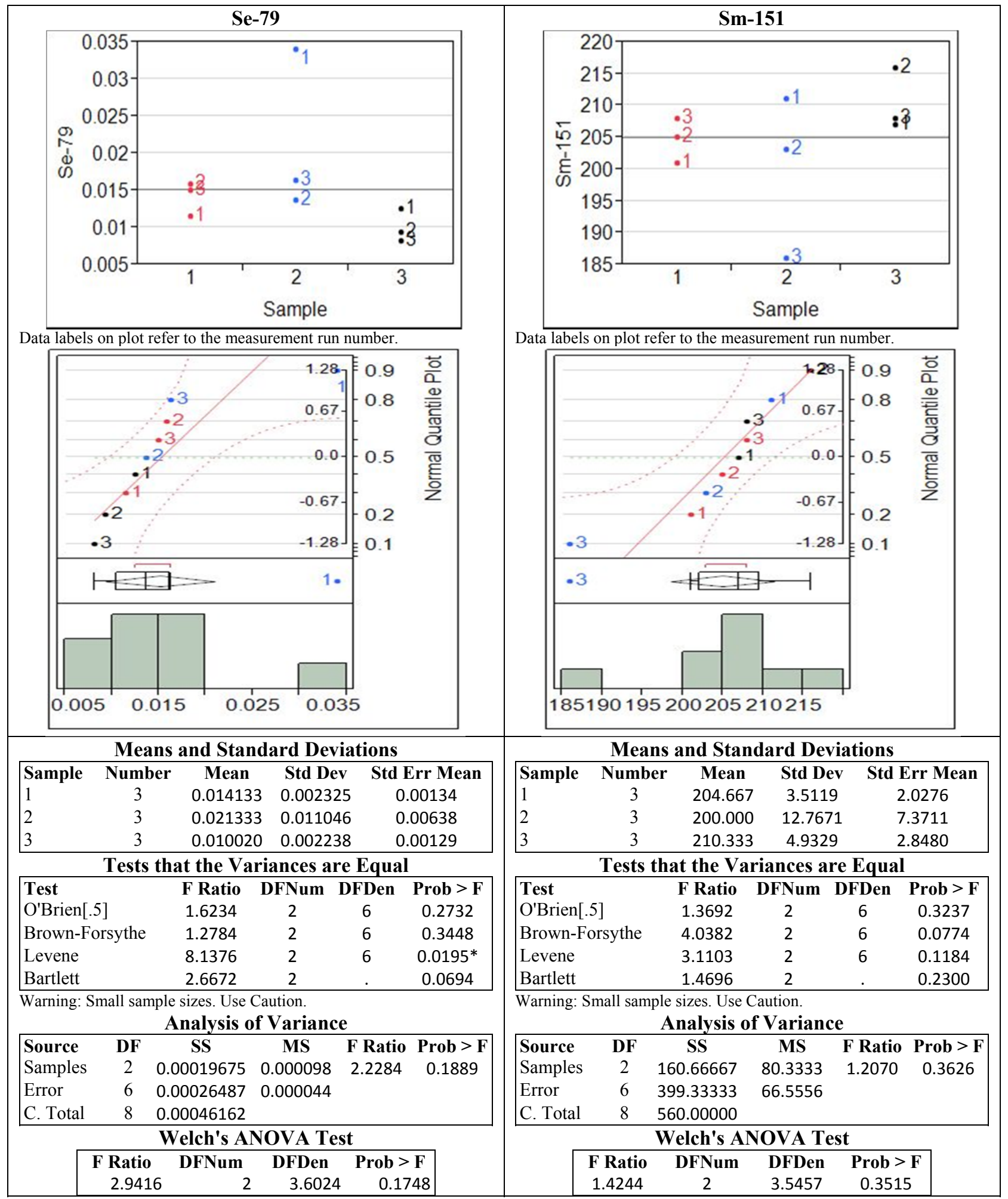




\section{Appendix C: Statistical Results for Radionuclide Constituents}

Table 25 Tests and Estimators for Radionuclides

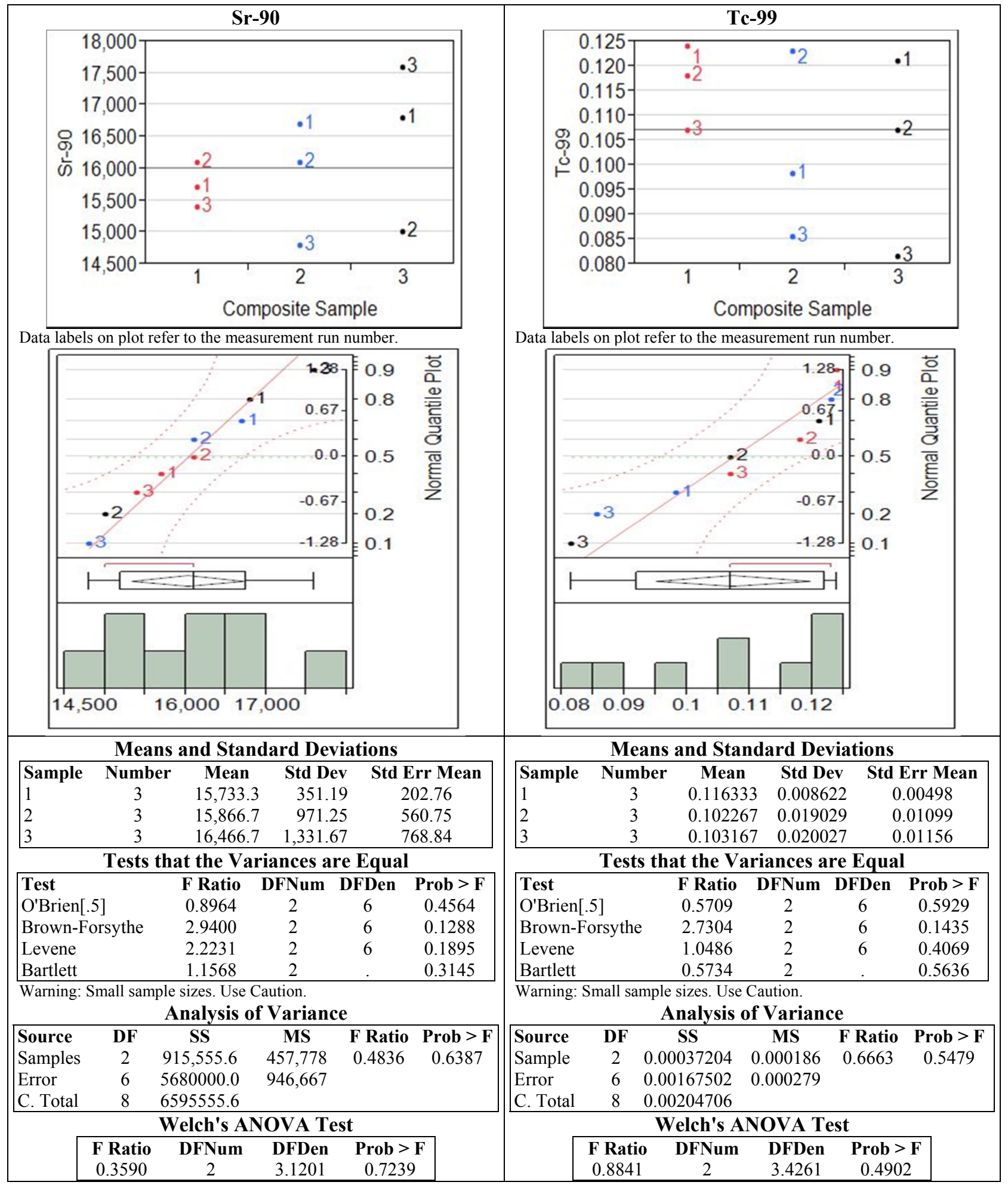




\section{Appendix C: Statistical Results for Radionuclide Constituents}

Table 25 Tests and Estimators for Radionuclides

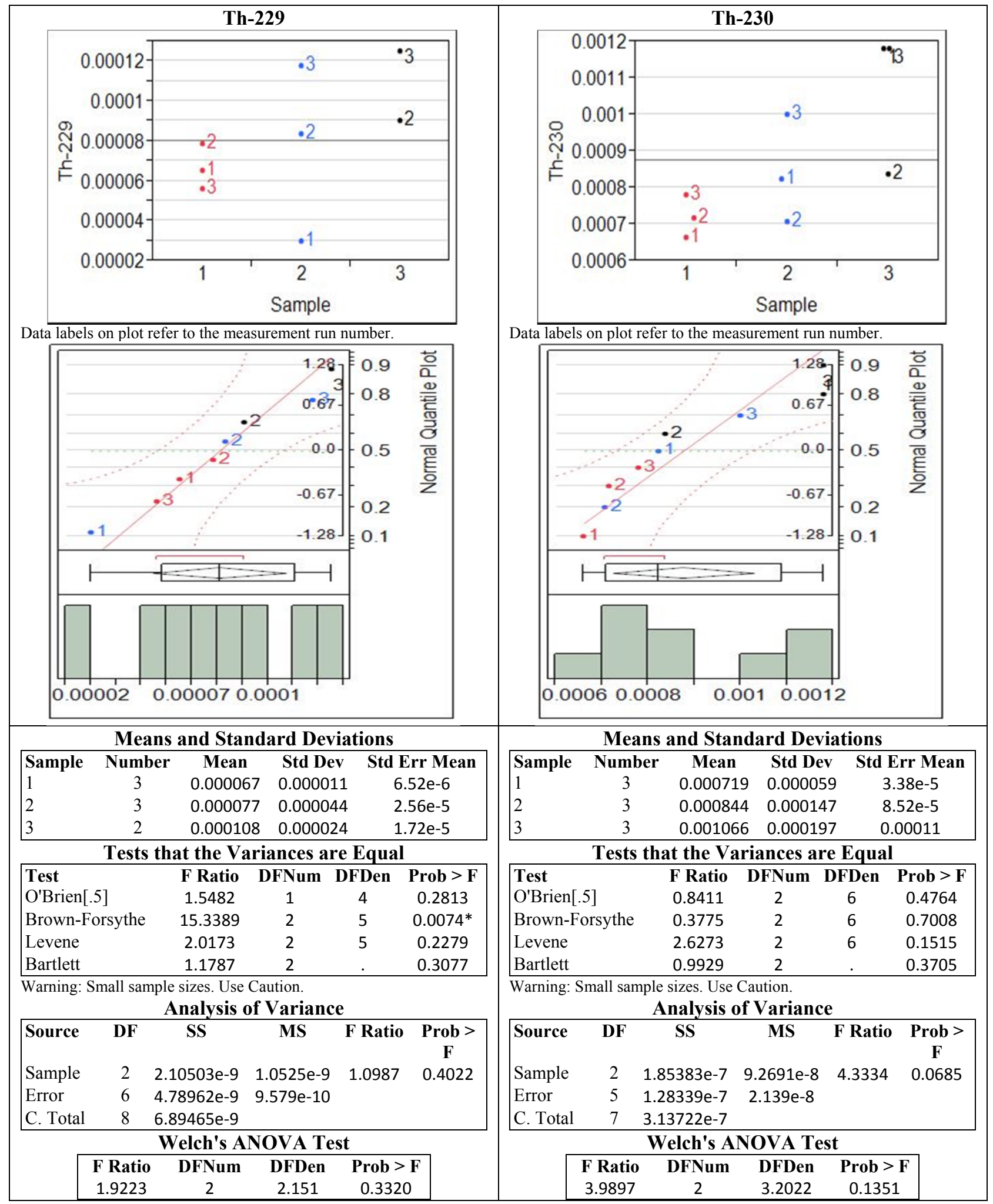




\section{Appendix C: Statistical Results for Radionuclide Constituents}

Table 25 Tests and Estimators for Radionuclides

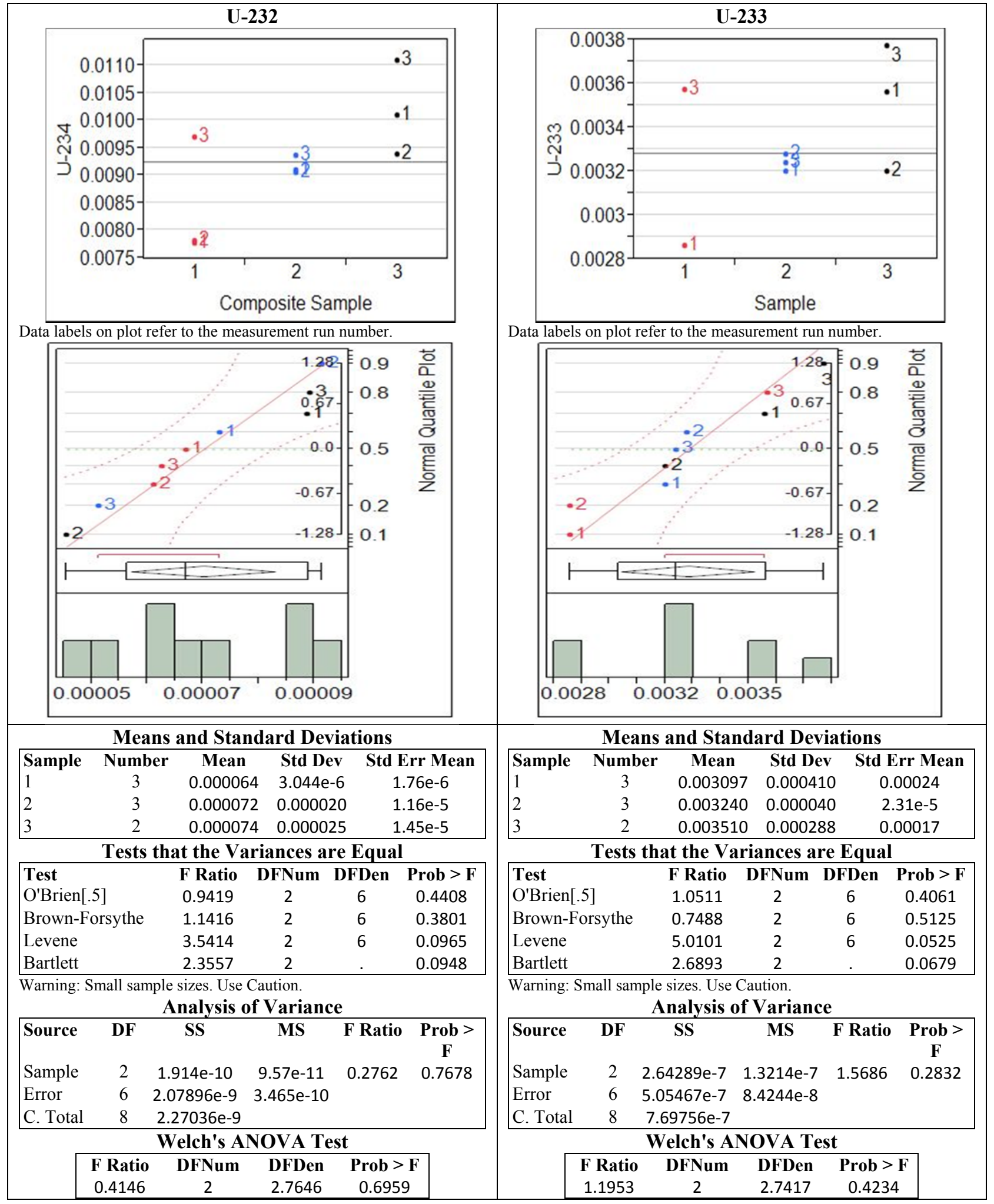




\section{Appendix C: Statistical Results for Radionuclide Constituents}

Table 25 Tests and Estimators for Radionuclides

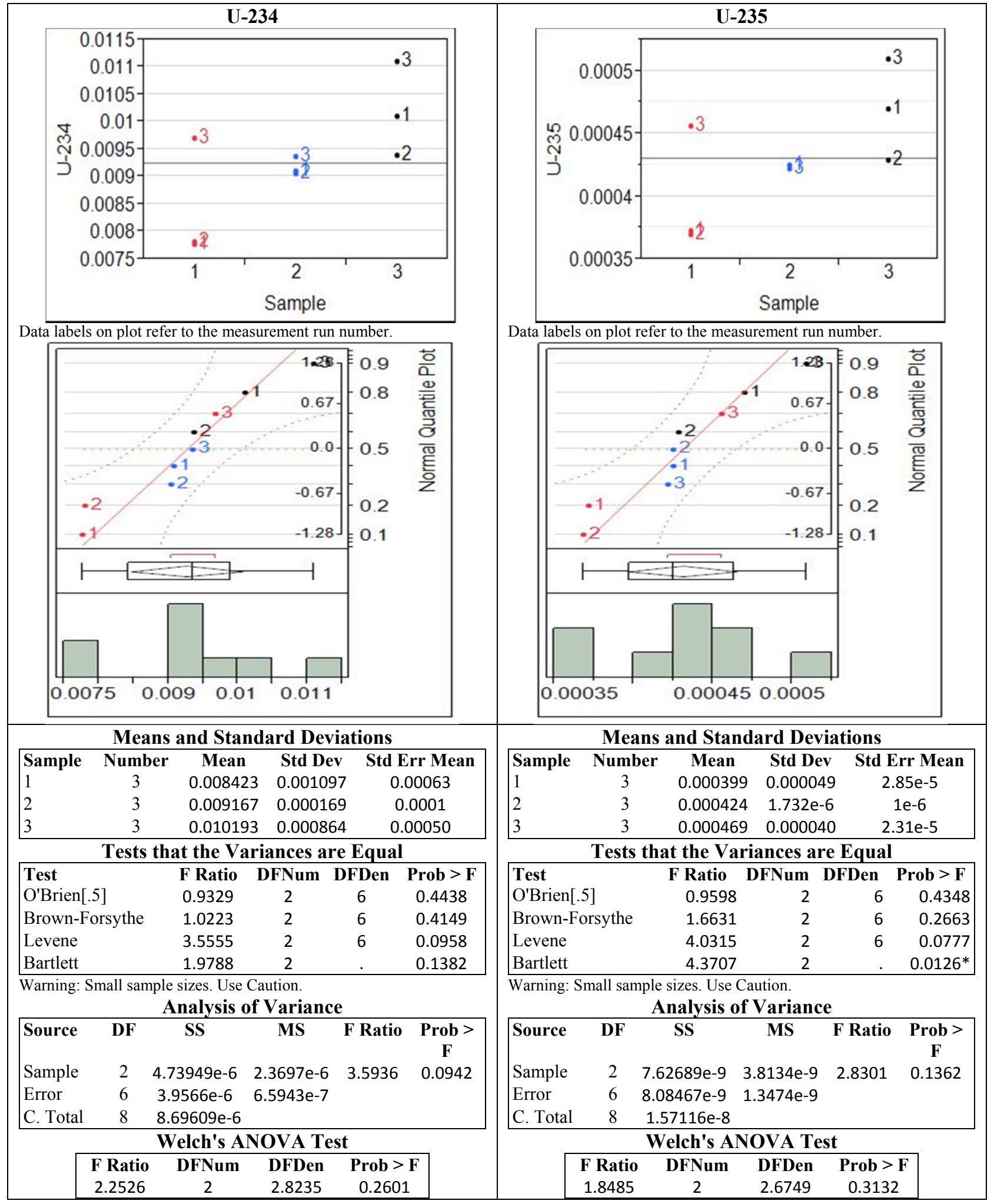




\section{Appendix C: Statistical Results for Radionuclide Constituents}

Table 25 Tests and Estimators for Radionuclides

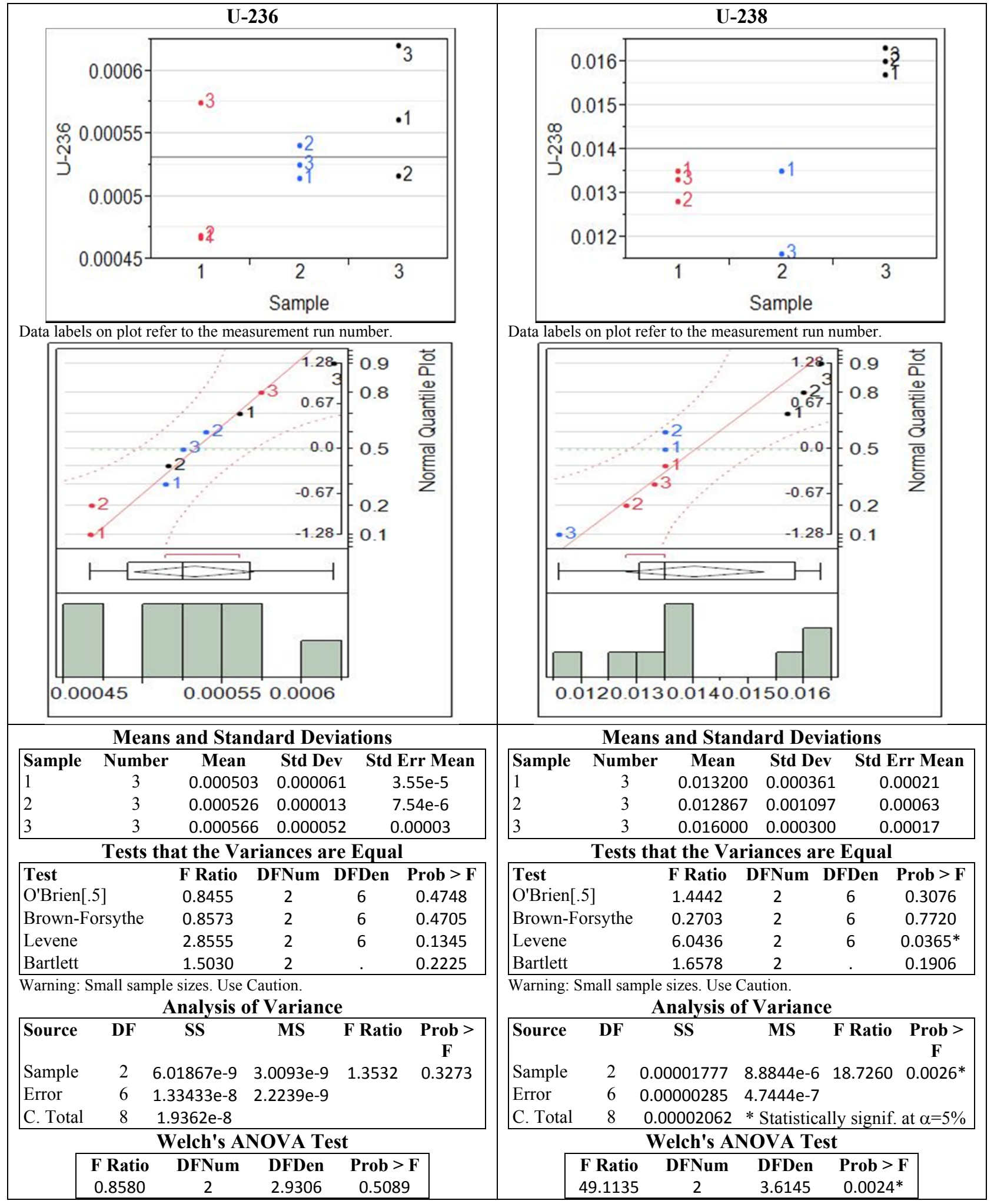




\section{Appendix C: Statistical Results for Radionuclide Constituents}

Table 25 Tests and Estimators for Radionuclides

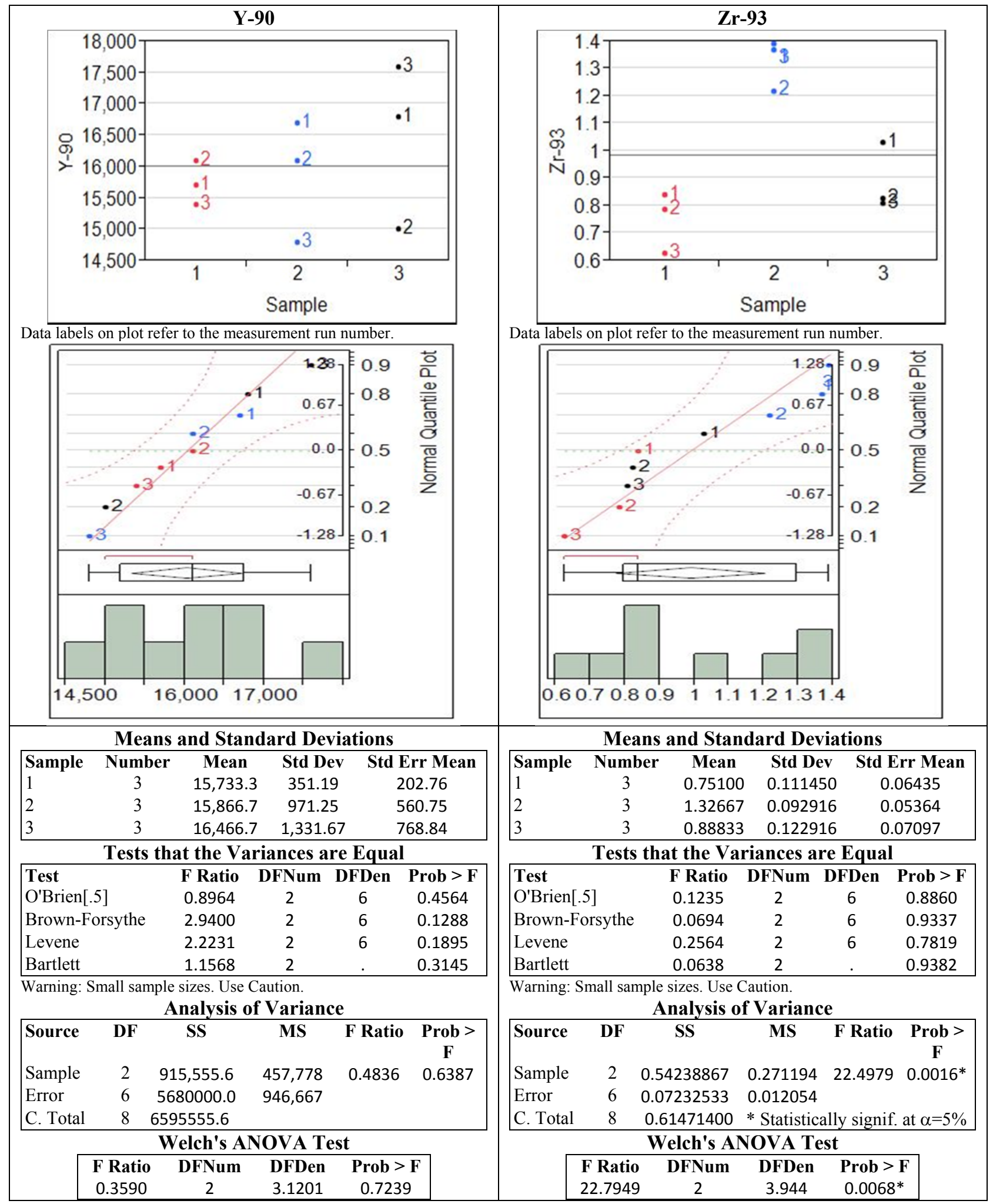




\section{Appendix C: Statistical Results for Radionuclide Constituents}

Table 26 Radionuclide Concentrations with All Results below Their MDCs

\begin{tabular}{|l|c|c|}
\hline & \multicolumn{2}{|c|}{ MDCs } \\
\hline & Min & Max \\
\hline Ac-227 & $7.21 \mathrm{E}-06$ & $4.59 \mathrm{E}-05$ \\
\hline $\mathrm{Cf}-249$ & $4.38 \mathrm{E}-03$ & $9.95 \mathrm{E}-03$ \\
\hline $\mathrm{Cm}-243$ & $5.18 \mathrm{E}-01$ & $1.45 \mathrm{E}+00$ \\
\hline $\mathrm{Cm}-247$ & $1.62 \mathrm{E}-07$ & $2.76 \mathrm{E}-07$ \\
\hline $\mathrm{Cm}-248$ & $8.33 \mathrm{E}-06$ & $2.39 \mathrm{E}-05$ \\
\hline Eu-152 & $1.46 \mathrm{E}-01$ & $1.91 \mathrm{E}-01$ \\
\hline $\mathrm{H}-3$ & $2.00 \mathrm{E}-02$ & $2.17 \mathrm{E}-02$ \\
\hline Nb-94 & $1.01 \mathrm{E}-03$ & $2.15 \mathrm{E}-03$ \\
\hline Pa-231 & $4.08 \mathrm{E}-04$ & $1.02 \mathrm{E}-03$ \\
\hline Pu-244 & $5.75 \mathrm{E}-07$ & $7.89 \mathrm{E}-07$ \\
\hline Ra-226 & $2.55 \mathrm{E}-03$ & $4.35 \mathrm{E}-03$ \\
\hline Sb-126 & $9.41 \mathrm{E}-02$ & $1.17 \mathrm{E}-01$ \\
\hline Sb-126m & $9.41 \mathrm{E}-02$ & $1.17 \mathrm{E}-01$ \\
\hline Sn-126 & $6.26 \mathrm{E}-01$ & $7.97 \mathrm{E}-01$ \\
\hline
\end{tabular}


Appendix C: Statistical Results for Radionuclide Constituents

Table 27 95\% UCLs for Mean Concentrations of Radionuclide Constituents with All Results above Their MDCs

\begin{tabular}{|c|c|c|c|c|c|c|}
\hline Radionuclide & Model Type & $\mathbf{N}$ & Mean & $\begin{array}{l}\text { Standard } \\
\text { Deviation }\end{array}$ & UCL95 & Notes \\
\hline Am-241 & Sampling Error & 9 & $6.848 \mathrm{e}+1$ & $1.182 \mathrm{e}+1$ & $8.767 \mathrm{e}+1$ & Normal \\
\hline Am-242m & No Sampling Error & 9 & $1.169 \mathrm{e}-1$ & $1.692 \mathrm{e}-2$ & $1.274 \mathrm{e}-1$ & Normal: Student's t UCL \\
\hline Am-243 & Sampling Error & 9 & $1.471 \mathrm{e}+0$ & $3.539 \mathrm{e}-1$ & $2.054 \mathrm{e}+0$ & Normal \\
\hline $\mathrm{Ba}-137 \mathrm{~m}$ & No Sampling Error & 9 & $5.132 \mathrm{e}+2$ & $1.785 \mathrm{e}+1$ & $5.243 \mathrm{e}+2$ & No discernable dist: Student's t UCL \\
\hline $\mathrm{C}-14$ & No Sampling Error & 9 & $1.784 \mathrm{e}-2$ & $5.299 \mathrm{e}-3$ & $2.113 \mathrm{e}-2$ & Normal: Student's t UCL \\
\hline $\mathrm{Cm}-244$ & Sampling Error & 9 & $5.544 \mathrm{e}+1$ & $8.147 \mathrm{e}+0$ & $6.855 \mathrm{e}+1$ & Normal \\
\hline $\mathrm{Cm}-245$ & Sampling Error & 9 & $5.476 \mathrm{e}-3$ & $8.924 \mathrm{e}-4$ & $6.897 \mathrm{e}-3$ & Normal \\
\hline Co-60 & Sampling Error & 9 & $7.557 \mathrm{e}+0$ & $8.023 \mathrm{e}-1$ & $8.862 \mathrm{e}+0$ & Normal \\
\hline Cs-135 & No Sampling Error & 9 & $2.743 e-3$ & $1.348 \mathrm{e}-4$ & $2.827 \mathrm{e}-3$ & Normal: Student's t UCL \\
\hline Cs-137 & No Sampling Error & 9 & $5.428 \mathrm{e}+2$ & $1.891 \mathrm{e}+1$ & $5.545 \mathrm{e}+2$ & No discernable dist: Student's t UCL \\
\hline Eu-154 & Sampling Error & 9 & $1.766 \mathrm{e}+1$ & $2.620 \mathrm{e}+0$ & $2.202 \mathrm{e}+1$ & Normal \\
\hline I-129 & Sampling Error & 9 & $1.593 \mathrm{e}-4$ & $2.819 \mathrm{e}-5$ & $2.047 \mathrm{e}-4$ & Normal \\
\hline $\mathrm{Ni}-59$ & No Sampling Error & 9 & $4.410 \mathrm{e}+0$ & $1.048 \mathrm{e}+0$ & $5.060 \mathrm{e}+0$ & Normal: Student's t UCL \\
\hline Ni-63 & No Sampling Error & 9 & $3.183 \mathrm{e}+2$ & $9.082 \mathrm{e}+1$ & $3.746 \mathrm{e}+2$ & Normal: Student's t UCL \\
\hline $\mathrm{Np}-237$ & Sampling Error & 9 & $1.981 \mathrm{e}-2$ & $8.064 \mathrm{e}-3$ & $3.191 \mathrm{e}-2$ & Normal \\
\hline $\mathrm{Pu}-238$ & No Sampling Error & 9 & $3.312 \mathrm{e}+0$ & $2.748 \mathrm{e}-1$ & $3.483 \mathrm{e}+0$ & Normal: Student's t UCL \\
\hline $\mathrm{Pu}-239$ & No Sampling Error & 9 & $2.916 \mathrm{e}+0$ & $1.907 \mathrm{e}-1$ & $3.034 \mathrm{e}+0$ & Normal: Student's t UCL \\
\hline $\mathrm{Pu}-240$ & No Sampling Error & 9 & $1.814 \mathrm{e}+0$ & $1.250 \mathrm{e}-1$ & $1.892 \mathrm{e}+0$ & Normal: Student's t UCL \\
\hline $\mathrm{Pu}-241$ & No Sampling Error & 9 & $6.738 \mathrm{e}+0$ & $4.636 \mathrm{e}-1$ & $7.025 \mathrm{e}+0$ & Normal: Student's t UCL \\
\hline $\mathrm{Pu}-242$ & No Sampling Error & 9 & $2.184 \mathrm{e}-3$ & $1.290 \mathrm{e}-4$ & $2.264 \mathrm{e}-3$ & Normal: Student's t UCL \\
\hline
\end{tabular}




\section{Appendix C: Statistical Results for Radionuclide Constituents}

Table 27 Continued. 95\% UCLs for Mean Concentrations of Radionuclide Constituents with All Results above Their MDCs

\begin{tabular}{|l|c|c|c|c|c|l|}
\hline Radionuclide & Model Type & $\mathbf{N}$ & Mean & Standard Deviation & UCL95 & \multicolumn{1}{|c|}{ Notes } \\
\hline Se-79 & No Sampling Error & 9 & $1.516 \mathrm{e}-2$ & $7.596 \mathrm{e}-3$ & $2.028 \mathrm{e}-2$ & Gamma: Gamma UCL \\
\hline Sm-151 & No Sampling Error & 9 & $2.050 \mathrm{e}+2$ & $8.367 \mathrm{e}+0$ & $2.102 \mathrm{e}+2$ & Normal: Student's t UCL \\
\hline Sr-90 & No Sampling Error & 9 & $1.602 \mathrm{e}+4$ & $9.080 \mathrm{e}+2$ & $1.659 \mathrm{e}+4$ & Normal: Student's t UCL \\
\hline Tc-99 & No Sampling Error & 9 & $1.073 \mathrm{e}-1$ & $1.600 \mathrm{e}-2$ & $1.172 \mathrm{e}-1$ & Normal: Student's t UCL \\
\hline Th-229 & No Sampling Error & 8 & $8.079 \mathrm{E}-5$ & $3.138 \mathrm{e}-5$ & $1.018 \mathrm{e}-4$ & Normal: Student's t UCL \\
\hline Th-230 & No Sampling Error & 9 & $8.762 \mathrm{e}-4$ & $1.980 \mathrm{e}-4$ & $9.990 \mathrm{e}-4$ & Normal: Student's t UCL \\
\hline U-232 & No Sampling Error & 9 & $7.002 \mathrm{e}-5$ & $1.685 \mathrm{e}-5$ & $8.046 \mathrm{e}-5$ & Normal: Student's t UCL \\
\hline U-233 & No Sampling Error & 9 & $3.282 \mathrm{e}-3$ & $3.102 \mathrm{e}-4$ & $3.474 \mathrm{e}-3$ & Normal: Student's t UCL \\
\hline U-234 & No Sampling Error & 9 & $9.261 \mathrm{e}-3$ & $1.043 \mathrm{e}-3$ & $9.907 \mathrm{e}-3$ & Normal: Student's t UCL \\
\hline U-235 & No Sampling Error & 9 & $4.308 \mathrm{e}-4$ & $4.432 \mathrm{e}-5$ & $4.582 \mathrm{e}-4$ & Normal: Student's t UCL \\
\hline U-236 & No Sampling Error & 9 & $5.317 \mathrm{e}-4$ & $4.920 \mathrm{e}-5$ & $5.622 \mathrm{e}-4$ & Normal: Student's t UCL \\
\hline U-238 & Sampling Error & 9 & $1.402 \mathrm{e}-2$ & $1.810 \mathrm{e}-3$ & $1.692 \mathrm{e}-2$ & Normal \\
\hline Y-90 & No Sampling Error & 9 & $1.602 \mathrm{e}+4$ & $9.080 \mathrm{e}+2$ & $1.659 \mathrm{e}+4$ & Normal: Student's t UCL \\
\hline Zr-93 & Sampling Error & 9 & $9.887 \mathrm{e}-1$ & $3.137 \mathrm{e}-1$ & $1.496 \mathrm{e}+0$ & Normal \\
\hline
\end{tabular}

Notes: All sampling error models were fitted in JMP® statistical software. The no sampling error models were fit in ProUCL 4.1 